\title{
Scanning AC Nanocalorimetry and Its Applications
}

\section{Citation}

Xiao, Kechao. 2015. Scanning AC Nanocalorimetry and Its Applications. Doctoral dissertation, Harvard University, Graduate School of Arts \& Sciences.

\section{Permanent link}

http://nrs.harvard.edu/urn-3:HUL.InstRepos:23845069

\section{Terms of Use}

This article was downloaded from Harvard University's DASH repository, and is made available under the terms and conditions applicable to Other Posted Material, as set forth at http:// nrs.harvard.edu/urn-3:HUL.InstRepos:dash.current.terms-of-use\#LAA

\section{Share Your Story}

The Harvard community has made this article openly available.

Please share how this access benefits you. Submit a story.

Accessibility 


\title{
Scanning AC Nanocalorimetry and Its Applications
}

\author{
A dissertation presented \\ by

\section{Kechao Xiao}

to

The School of Engineering and Applied Sciences

in partial fulfillment of the requirements

\author{
for the degree of \\ Doctor of Philosophy \\ in the subject of \\ Engineering Sciences
}

Harvard University

Cambridge, Massachusetts

June 2015 
(C) 2015 Kechao Xiao

All rights reserved. 


\title{
Scanning AC Nanocalorimetry and Its Applications
}

\begin{abstract}
This thesis presents an AC nanocalorimetry technique that enables calorimetry measurements on very small quantities of materials over a wide range of scanning rates (from isothermal to $3 \times 10^{\wedge} 3 \mathrm{~K} / \mathrm{s}$ ), temperatures (up to $1200 \mathrm{~K}$ ), and environments. Such working range bridges the gap between traditional scanning calorimetry of bulk materials and nanocalorimetry. The method relies on a micromachined nanocalorimeter with negligible thermal lags between heater, thermometer, and sample. The ability to perform calorimetry measurements over such a broad range of scanning rates makes it an ideal tool to characterize the kinetics of phase transformations, reactions at elevated temperatures or to explore the behavior of materials far from equilibrium. We demonstrate the technique by performing measurements on thin-film samples of Sn, In, and $\mathrm{Bi}$ with thicknesses ranging from 100 to $300 \mathrm{~nm}$. The experimental heat capacities and melting temperatures agree well with literature values. The measured heat
\end{abstract}


capacities are insensitive to the applied AC frequency, scan rate, and heat loss to the environment over a broad range of experimental conditions.

The dynamic range of scanning $\mathrm{AC}$ nanocalorimetry enables the combination of nanocalorimetry with in-situ $x$-ray diffraction (XRD) to facilitate interpretation of the calorimetry measurements. Time-resolved XRD during in-situ operation of nanocalorimetry sensors using intense, high-energy synchrotron radiation allows unprecedented characterization of thermal and structural material properties. We demonstrate this experiment with detailed characterization of the melting and solidification of elemental $\mathrm{Bi}, \mathrm{In}$ and $\mathrm{Sn}$ thin-film samples, using heating and cooling rates up to $300 \mathrm{~K} / \mathrm{s}$.

By combining scanning DC and AC nano-calorimetry techniques, we study the nucleation behavior of undercooled liquid $\mathrm{Bi}$ at cooling rates ranging from $10^{\wedge} 1$ to $10^{\wedge} 4$ $\mathrm{K} / \mathrm{s}$. Upon initial melting, the Bi thin-film sample breaks up into isolated islands. The number of islands in a typical sample is sufficiently large that highly repeatable nucleation behavior is observed, despite the stochastic nature of the nucleation process. We establish a data reduction technique to evaluate the nucleation rate from $D C$ and $A C$ calorimetry results. The results show that the driving force for the nucleation of melted $\mathrm{Bi}$ is well described by classical nucleation theory over a wide range of cooling rates. The proposed technique provides a unique and efficient way to examine nucleation kinetics 
with cooling rates over several orders of magnitude. The technique is quite general and can be used to evaluate reaction kinetics in other materials.

Lastly, we apply the scanning AC nanocalorimetry technique to study solid-gas phase reactions by measuring the change in heat capacity of a sample during reaction. We apply this approach to evaluate the oxidation kinetics of thin-film samples of zirconium in air. The results confirm parabolic oxidation kinetics with an activation energy of $0.59 \pm 0.03 \mathrm{eV}$. The nano-calorimetry measurements were performed using a device that contains an array of micromachined nano-calorimeter sensors in an architecture designed for combinatorial studies. We demonstrate that the oxidation kinetics can be quantified using a single sample, thus enabling high-throughput mapping of the composition-dependence of the reaction rate. 


\section{Table of Contents}

Title Page ..................................................................................................................

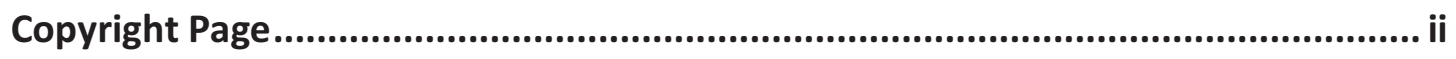

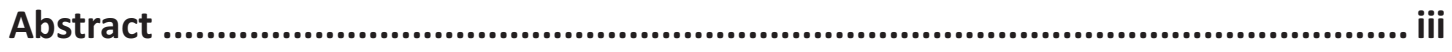

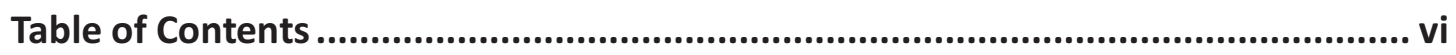

Acknowledgement ................................................................................................. ix

Chapter 1 Introduction ................................................................................... 1

1.1 Scanning calorimetry and nanocalorimetry ................................................ 1

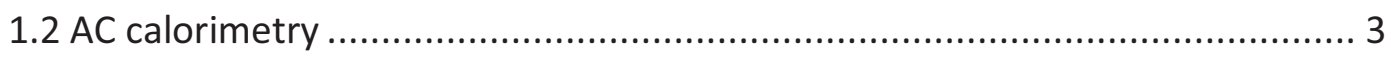

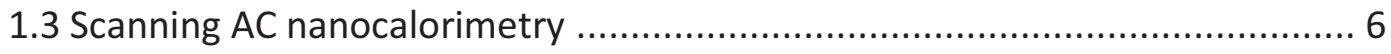

1.4 The goal and outline of this thesis............................................................

Chapter 2 A scanning AC calorimetry technique for the analysis of nano-quantities of

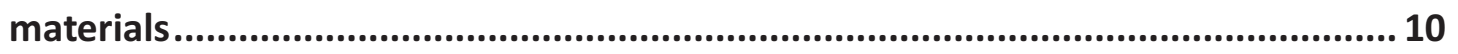

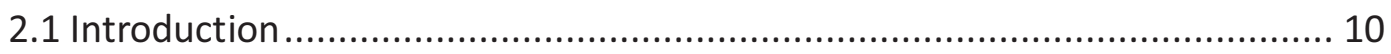

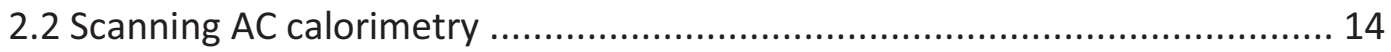

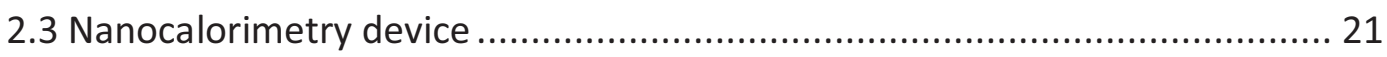

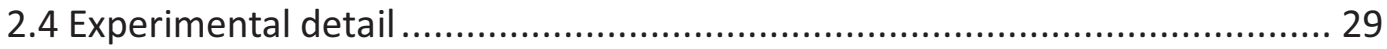

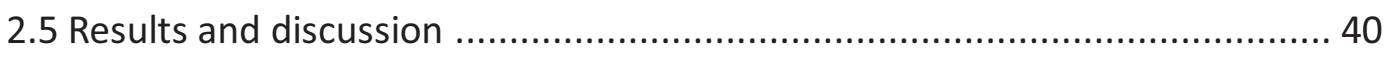

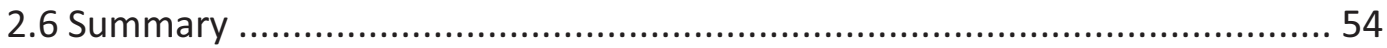

Chapter 3 Scanning AC nanocalorimetry combined with in-situ x-ray diffraction... 55

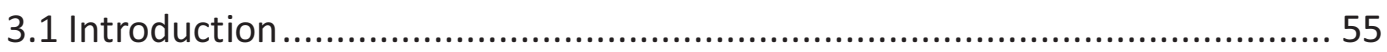




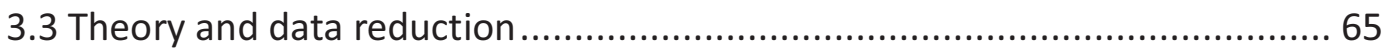

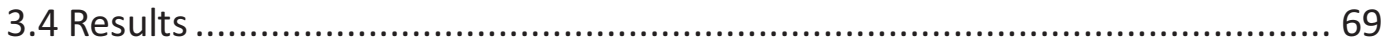

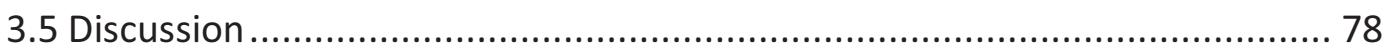

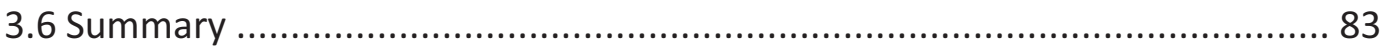

Chapter 4 Nucleation behavior of melted Bi films at cooling rates from $10^{\wedge} 1$ to $10^{\wedge} 4$ $\mathrm{K} / \mathrm{s}$ studied by combining scanning $\mathrm{AC}$ and $\mathrm{DC}$ nano-calorimetry techniques ........ 84

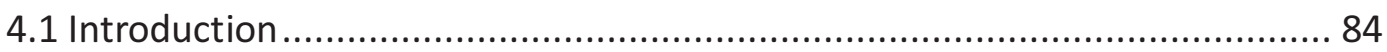

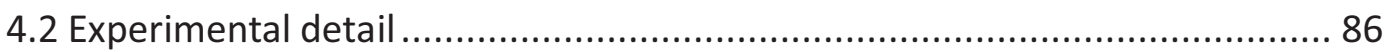

4.3 Analysis of reaction kinetics by combining DC and scanning AC measurement results.............................................................................. 90

4.4 Application to nucleation theory ................................................... 95

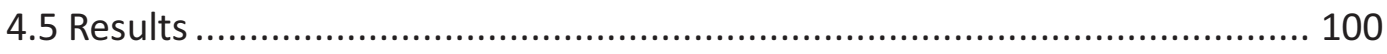

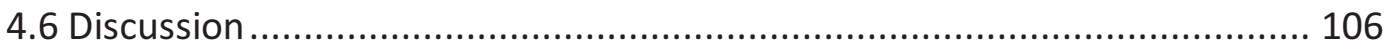

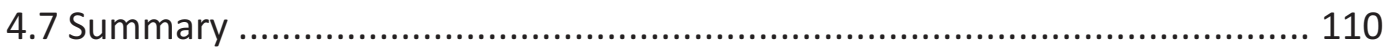

Chapter 5 Kinetics of solid-gas reactions characterized by scanning AC nano-calorimetry with application to $\mathrm{Zr}$ oxidation ....................................... 112

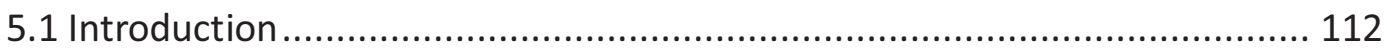

5.2 Experimental details and results .................................................. 114

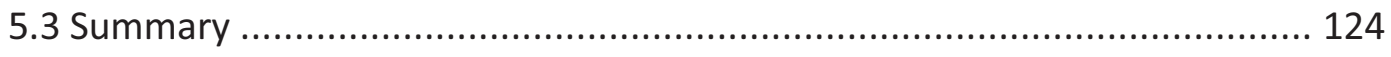

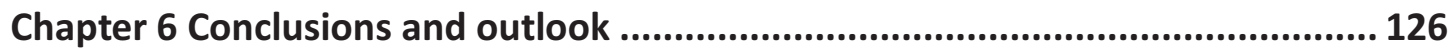

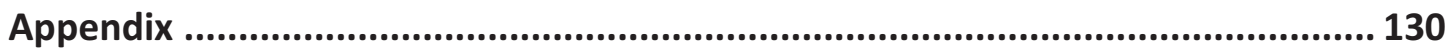

Appendix A: Validity of AC measurements (Chapter 2) ............................. 130 
Appendix B: Derivation of equations for the $\varphi_{1}$ and $C$ (Chapter 3) ................. 132

Appendix C: Data reduction scheme for DC measurements (Chapter 4) .......... 134

Appendix D: Effect of size distribution on the nucleation rate (Chapter 4) ........ 137

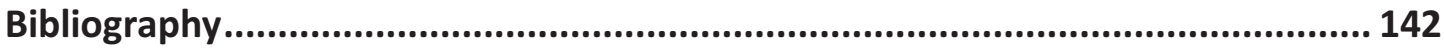




\section{Acknowledgement}

As I start to write this section, I cannot help to look back on my five years life at Harvard. It has been five years full of challenges, learnings and rewards, and I am deeply indebted to the people mentioned here for their generous support.

I am most grateful to my advisor, Professor Joost Vlassak, for his continuous guidance, encouragement and support. He led me into the world of scientific research and equipped me with the knowhow to start my own journey. He is knowledgeable, creative and always ready to help. He has been my turn-to person whenever I need advice. For countless times, I would walk in his office full of confusion and walk out half an hour later with a clear picture and path in mind. From him, I learned not only knowledge, but also the way of critical thinking and problem solving. I feel extremely lucky to have him as my PhD advisor.

I would also like to thank my dissertation committee, Professor Frans Spaepen, Professor David Clarke and Professor Katia Bertoldi for their invaluable suggestions and comments throughout my research. Specifically, Frans was a great resource to turn to when I was trying to understand the nucleation of metals. David is an expert in the oxidation of materials, and answered many of my related questions. Katia's class, ES $242 r$, trained me on the Finite Element Method, which is crucial in my design of new devices. 
I am indebted to my senior labmates Dr. Patrick McCluskey and Dr. John Gregoire, whom I had the privilege to work with when I first joined the group. Patrick is one of the pioneers in combinatorial nanocalorimetry. With great patience, he introduced to me the basics of nanocalorimetry and the related experimental skills. John is incredibly knowledgeable and versatile. Apart from being a researcher, he is also an experienced mechanic, electrician and programmer. He has designed and built many of our key experimental equipment, and he seems to be capable of fixing just about anything. Above all, he is a super nice person, always there to lend his hand. The three of us once worked together in the dungeon of Cornell High Energy Synchrotron Source (CHESS) for seven days straight with little sleep. That is one of my most memorable moments in graduate school.

Special thanks to Vlassak group members, Dr. Jianyu Li, Dr. Gidong Sim, Dr. Kamyar Davoudi, Dr. Gayatri Cuddalorepatta, Dr. Widusha R.K. Illeperuma, Dr. George (Matt) Pharr, Yong Seok Choi, Dr. Xiaodong Zhang, Dr. Han Li, Dr. Xi Wang, Dongwoo Lee, Lauren Hartle, Yanhui Chu, Kevin Tian, Dr. Xin Wang, Dr. Libin Gao, Haitao Zhang and Lu Wu. In particular, Jainyu has been my best buddy both at work and in life. Gidong, Dongwoo, Gayatri and Yanhui have been wonderful collaborators. All the people here are good friends. We have fun together and we help each other.

I would also like to extend thanks to my colleagues and friends from Harvard: Dr. 
Samuel Shian, Dr. Andrej Kosmrlj, Qihan Liu, Jia Liu, Sicong Shan, Pai Wang, Paul Loschak, Helen Wu, Hao Pei, Dr. Yu Qin, Dr. Tianxiang Su, Yang He and many others. These people support me through my time here.

Finally, I would like to dedicate this thesis to my loving family. They have been my biggest support all along the way. My parents have been my best teachers, role models and friends. They raised me up with unconditional love and showed me how to be kind, considerate and honest. They have encouraged me to pursue my dreams, and they will not hesitate to give me their best support whenever I am in need. And my dear wife, Miao. You have supported me with love, encouragement and trust through all of my ups and downs. You are caring, intelligent and lively, always bringing in fun and happiness. You are the sun-shine of my life. So are you, Yanda, my beloved son. Your smile is the best cure for my tiredness and the best motivation for me to keep going. One day you will be able to read this thesis, and just thinking about that makes me proud. 


\section{Chapter 1}

\section{Introduction}

\subsection{Scanning calorimetry and nanocalorimetry}

Scanning calorimetry is an important tool in materials research [1]. Valuable insight is gained in the behavior of a material by measuring the heat absorbed or released when heating or cooling that material. This technique has long been used to determine phase transformation temperatures, enthalpy of reactions, transformation kinetics and heat capacities. In a typical calorimetry measurement, power is supplied to the calorimeter cell, changing the temperature of the sample. Under isobaric conditions, the energy balance equation for such a measurement can be written as

$$
P+\dot{H}=C \dot{T}+L
$$

where $P$ is the input power, $C$ is the heat capacity of the sample and the calorimeter addendum, $\dot{T}$ is the scanning rate of temperature, $\dot{H}$ is the rate of enthalpy production associated with a phase transition or chemical reaction, taken positive for an exothermic process, and $L$ is the rate of heat loss to the environment. The goal of the measurement is to determine $\dot{H}$ and $C$ as a function of temperature. Generally, the heat loss $L$ cannot be measured directly, and is either minimized and neglected, as in the case of adiabatic calorimetry, or eliminated from the equation by making differential 
measurements, as in the case of differential scanning calorimetry.

Nanometer-sized materials are of tremendous interest in both scientific and technological communities. Continued development of integrated circuits, storage devices, sensors, and micro-electro-mechanical system increases the need to understand thermal properties of materials at reduced length scales. Traditional calorimetry has been well developed for bulk materials, but is not sensitive enough to perform measurements on nanoscale samples. Nanocalorimetry is a novel technique that has developed rapidly in recent years [2-10]. It enables ultrasensitive calorimetry measurements on very small samples of materials through use of micromachined sensors with vanishingly small thermal mass. The sensitivity of nanocalorimetry sensors makes it possible to perform quantitative measurements on thin-film samples with thickness as small as a few nanometers or particles with a mass as small as a few nanograms [11-14]. However, the small thermal mass also presents a problem - the measurements are very sensitive to heat loss. In fact, the effect of heat loss can be evaluated by its ratio to the heat capacity term in Eq. (1.1). The adiabatic condition requires that

$$
\frac{L}{C \dot{T}} \propto \frac{S L_{s}(T)}{V \dot{T}} \propto \frac{L_{s}(T)}{l \dot{T}}<<1,
$$

where $L_{s}(T)$ represents an increasing function of the temperature difference between sample and environment. In Eq. (1.2), the heat loss is proportional to the surface area, $S$, 
of the sample, while the heat capacity of the sample is proportional to its volume, $V$. Thus, the effect of heat loss increases inversely with sample dimension I and scanning rate $\dot{T}$.

In bulk scanning calorimetry, the scan rate is typically limited to a maximum on the order of $10 \mathrm{~K} / \mathrm{min}$ [1], because of the relatively large samples used in these measurements. Nanocalorimetry, in contrast, can achieve very fast scanning rates due to the small thermal mass of both sensor and sample, but fast scanning rates are also a necessity because otherwise heat losses to the environment become unacceptably large. For instance, the nanocalorimetry sensor originally developed by Allen and coworkers [15] can attain heating rates as large as $10^{5} \mathrm{~K} / \mathrm{s}$, but is lower bounded by $10^{4} \mathrm{~K} / \mathrm{s}$ to achieve the adiabatic condition (Eq. (3)) [15]. Schick et al have developed a sensor that can operate under non-adiabatic conditions with controlled heating and cooling rates in the range of $10^{3} \mathrm{~K} / \mathrm{s}[16]$, but its accuracy degrades at lower heating rates.

\subsection{AC calorimetry}

AC calorimetry is well known as a sensitive technique for measuring thermo-physical properties of materials in the presence of heat loss [17-20]. In AC calorimetry, an oscillating heat input is supplied to the sample and the resulting temperature oscillation is measured [20]. In its simplest form, the power heating the sample is a constant plus a 
sine wave. Equation (1.1) then reads

$$
P_{0}+P_{1} \sin \omega t+\dot{H}=C \dot{T}+L
$$

where $P_{0}$ and $P_{1}$ are slowly varying parameters and $\omega$ is the angular frequency of the oscillation. The AC power component causes the temperature of the sample to oscillate around an average temperature that slowly changes with time. The temperature response of the sample can then be written as

$$
T=T_{0}(t)+\theta(t)
$$

where $T_{0}(t)$ is the temperature averaged over one oscillation period, and $\theta(t)$ represents the oscillating part of the temperature. If both $\dot{H}$ and $L$ are functions of temperature, the oscillatory component of the temperature is

$$
\begin{aligned}
& \theta(t)=\theta_{0} \sin (\omega t-\varphi), \\
& \theta_{0}=\frac{P_{1} \sin \varphi}{\omega C}, \\
& \tan \varphi=\omega C /\left.\frac{\partial(L-\dot{H})}{\partial T}\right|_{T_{0}} .
\end{aligned}
$$

According to Eq. (1.6), the heat capacity, $C$, of the sample and addendum can be determined directly from a measurement of the amplitude and phase lag of the oscillatory temperature response. Furthermore, Equation (1.7) shows that the phase angle provides information on the temperature dependence of the heat loss and the rate of enthalpy production. When the frequency is large enough that $\tan \varphi \gg 1$, the phase angle $\varphi$ approaches $\pi / 2$. Under this condition, the correction for heat loss is 
insignificant and the heat capacity can be calculated directly from the amplitude of the temperature oscillations.

The maximum scan rate and frequency that can be used in an AC calorimetry measurement depend on the geometry and size of the calorimeter. The thermal wave associated with the AC component of the power decays over the thermal diffusion length $\eta=\sqrt{K_{m} /(\omega C)}$, where $K_{m}$ is the thermal conductivity of the medium in which the wave propagates [21]. Consequently, the volume of material probed by the thermal wave scales with $\eta$ and decreases inversely with $\omega$. To ensure a well-defined sample size, $\eta$ should be larger than the size of the sample. Furthermore, the separation distance between sample, heater and thermometer results in the measured temperature oscillations being damped and delayed with respect to the temperature oscillations of sample and heater [6]. This effect is negligible if the separation is much smaller than $\eta$, but it increases rapidly as the separation exceeds $\eta$. If the frequency used in a measurement is sufficiently small, thermal effects do not affect the measurement; otherwise a thermal model of the calorimeter is needed to interpret the results. In a typical measurement on a bulk material [17], the frequency is kept below $1 \mathrm{~Hz}$ to prevent any thermal gradients. Because $T_{0}(t)$ should not change significantly during a single temperature oscillation, the low frequency also sets an upper limit of approximately one Kelvin per second on the scanning rate. In practice, the scanning 
rates for bulk $A C$ calorimetry are similar to that for bulk $D C$ calorimetry $(<10 \mathrm{~K} / \mathrm{min})$ to ensure temperature uniformity.

\subsection{Scanning AC nanocalorimetry}

Calorimetry measurements at very slow heating and cooling rates allow a material to remain at or near equilibrium and provide information on its thermodynamic properties, while measurements at faster scan rates provide characterization of reaction kinetics and materials far from equilibrium. The ability to make measurements up to ultra-high temperatures $(>1000 \mathrm{~K})$ is interesting also, as many reactions have noticeable rate only at elevated temperatures. Thus, a calorimetry technique that can operate over a broad working range is an attractive and powerful tool for materials researchers. However, as discussed above, there is a discrepancy between the fastest scanning rate for bulk calorimetry $(\sim 10 \mathrm{~K} / \mathrm{min})$ and the slowest scanning rate for nanocalorimetry $\left(\sim 10^{3} \mathrm{~K} / \mathrm{s}\right)$.

Scanning AC nanocalorimetry has been developed to enable AC nanocalorimetry measurements at heating and cooling rates that vary from isothermal to $3 \times 10^{3} \mathrm{~K} / \mathrm{s}$ (and possibly higher) [22]. The technique is developed and tested using the parallel nano scanning calorimeter (PnSC) $[7,23]$. The PnSC has a small thermal impedance between heater, thermometer, and sample, allowing $\mathrm{AC}$ frequencies up to $100 \mathrm{kHz}$ without the 
need for correction. This increased range of usable frequencies makes it possible to perform AC measurements at much faster scan rates than bulk AC calorimetry. Furthermore, it is no longer necessary to perform nanocalorimetry measurements at very fast scanning rates to minimize heat losses, because $A C$ measurements are virtually insensitive to heat loss. Thus, scanning AC nanocalorimetry enables calorimetry measurements under isothermal conditions as well as over a broad range of heating and cooling rates. In effect, the technique bridges the gap in working range between nanocalorimetry and bulk calorimetry. The technique can be applied to study a broad range of reactions, e.g. high temperature solid-gas reactions of thin-film materials, or the reaction kinetics under a wide range of scanning rates, just to name a few [24, 25]. Thanks to the wide time-scale of measurements, the technique can also be combined with in-situ X-ray diffraction (XRD) to obtain structural information of phase transition during calorimetry scans [26].

\subsection{The goal and outline of this thesis}

The goal of this thesis is to develop scanning AC nanocalorimetry, to understand its applicable range and limitations, and to explore its applications in the study of thin-film materials. It is organized as follows. Chapter 2 describes in details the fundamental principles of scanning AC calorimetry, its capabilities and limitations, as well as the 
experimental setups. Measurements on pure elements are performed at different scanning rates and $A C$ frequencies to demonstrate the validity of the technique. Chapters 3 to 5 consider 3 promising areas of applications. Chapter 3 introduces the combination of scanning AC nanocalorimetry with in-situ XRD. The two techniques generally operate at very different time scales ( $1 \mathrm{~ms}$ for nanocalorimetry and $\sim 1 \mathrm{~h}$ for $\mathrm{XRD})$. The application of scanning AC nanocalorimetry and the use of synchrotron X-ray source bring an overlap in the time-scale window, enabling the combination. In-situ XRD provides structural information of sample during calorimetry scan, elucidating the phase transitions corresponding to calorimetry signals. Chapter 4 explores the combination of both scanning $A C$ and $D C$ nanocalorimetry to achieve cooling rates ranging from $10^{1} \mathrm{~K} / \mathrm{s}$ to $10^{4} \mathrm{~K} / \mathrm{s}$ in the study of $\mathrm{Bi}$ nucleation. The combination relies on the ability of both techniques to evaluate the rate of reaction, a not so straightforward task especially for AC calorimetry. The achieved wide range of cooling rates is helpful to understanding nucleation. Chapter 5 studies the oxidation reaction of thin-film samples in air. Scanning AC nanocalorimetry is applied to measure the change in heat capacity of the sample during the oxidation reaction, which in turns provides the oxidation kinetics and the corresponding activation energy. The technique can also be applied to the study of a broad range of thin-film solid gas reactions. Due to the small size of thin-film samples and the high temperature of reaction, these reactions may not be easily studied 
otherwise. Chapter 6 summarizes the development of the scanning AC nanocalorimetry technique to date, and proposes potential future works. 


\section{Chapter 2}

\section{A scanning AC calorimetry technique for the analysis of nano-quantities of materials*}

\subsection{Introduction}

Scanning calorimetry is an important tool in materials research. Valuable insight is gained in the behavior of a material by measuring the heat absorbed or released when heating or cooling that material. This technique has long been used to determine phase transformation temperatures, enthalpies, and heat capacities. Measurements at very slow heating and cooling rates allow a material to remain at or near equilibrium and provide information on its thermodynamic properties, while measurements at faster scan rates provide characterization of reaction kinetics [27]. The ability to perform calorimetry measurements at very fast scan rates is interesting also, as it enables the study of metastable phases and more generally of materials far from equilibrium $[3,4$, 28-30]. Thus, a scanning calorimetry technique that can operate over a broad range of heating and cooling rates is an attractive and powerful tool for materials researchers.

Traditional scanning calorimetry is well developed. Measurements are performed on

\footnotetext{
${ }^{*}$ Reprinted with permission from K. Xiao, J. M. Gregoire, P. J. McCluskey and J. J. Vlassak, Review of
} Scientific Instruments 83 (11), 114901 (2012). Copyright 2012, AIP Publishing LLC 
bulk materials and provide very precise information on their thermal properties. The scan rate in traditional calorimetry is limited to a maximum on the order of $10 \mathrm{~K} / \mathrm{min}$, because of the relatively large sample mass required for these measurements. Nanocalorimetry, by contrast, is not bound by this limitation and can achieve much higher heating and cooling rates. Nanocalorimetry is a novel technique that has developed rapidly in recent years $[2,3]$. It enables ultrasensitive calorimetry measurements on very small samples of materials through use of micromachined sensors with vanishingly small thermal mass. The sensitivity of nanocalorimetry sensors makes it possible to perform measurements on thin-film samples and composition libraries [7]. Due to the small thermal mass of sensor and sample, very fast heating can be achieved. For instance, the nanocalorimetry sensor originally developed by Allen and coworkers [15] can attain heating rates as large as $10^{5} \mathrm{~K} / \mathrm{s}$, extending the maximum heating rate of traditional calorimetry by many orders of magnitude. However, the small thermal mass also presents a problem - the measurements are very sensitive to heat loss and the requirement of adiabatic measurement conditions limits the scan rate to this very fast regime. The fraction of input power that is lost to heat must be small, placing a lower bound on the heating rate, which is $10^{4} \mathrm{~K} / \mathrm{s}$ for the calorimetry sensor developed by Allen and colleagues [15]. Schick et al have developed a sensor that can operate under non-adiabatic conditions with controlled heating and cooling rates in the 
range of $10^{3} \mathrm{~K} / \mathrm{s}[16]$, but its accuracy degrades at lower heating rates.

The issue of heat loss in nanocalorimetry at slow scanning rates can be addressed through use of alternating current $(A C)$ techniques. AC calorimetry is well known as a sensitive technique for measuring thermo-physical properties of materials in the presence of heat loss [18-20], and is thus often applied in isothermal measurements. In AC calorimetry, an oscillating heat input is supplied to the sample and the resulting temperature oscillation is measured [20]. The amplitude and phase lag of the temperature response provide accurate information on both the heat capacity of the sample and its heat loss to the environment. Specifically, the out-of-phase component of the temperature oscillation provides information on the heat capacity of the sample, while the in-phase component is associated with heat losses and irreversible processes. If the oscillation frequency is high enough, the contribution of the heat loss vanishes and the measurement can be regarded as adiabatic [20]. When AC calorimetry is applied to bulk materials, the relevant frequency range is quite low $(\sim 1 \mathrm{~Hz})$, because of the finite size of the sample and the thermal resistance between the sample, heater, and thermometer [17]. Indeed, the low frequency increases the thermal diffusion length and ensures that the entire sample is subjected to the maximum temperature oscillation, essential to obtaining accurate heat capacity measurement. The low frequency also sets an upper limit on the scanning rate to ensure that the mean temperature of the sample 
does not change significantly during a single temperature oscillation [17].

When applied to nanocalorimetry, the reduced thermal time scales expand the applicable frequency range. Schick and colleagues have developed an AC calorimeter for measurements on nano-scale materials using a commercial sensor from Xensor Integration [31]. In this device, the applied AC frequency is limited to approximately $100 \mathrm{~Hz}$ because the distance between the sample and the temperature sensor dampens the temperature response of the sensor [32]. Moreover, the scan rate cannot exceed 5 $\mathrm{K} / \mathrm{min}$ because of considerations of temperature uniformity across the sample and accuracy of the temperature measurements [31].

In this paper, we present a scanning $A C$ nanocalorimetry method that enables $A C$ measurements at heating and cooling rates that vary from isothermal to $2 \times 10^{3} \mathrm{~K} / \mathrm{s}$ (and possibly higher) - in terms of scan rate, the technique bridges the gap between nanocalorimetry and traditional scanning calorimetry of bulk materials. To the best of our knowledge, it is the first method to raise the maximum scan rate for AC calorimetry to the $10^{3} \mathrm{~K} / \mathrm{s}$ level. The method is developed and tested using the parallel nano scanning calorimeter (PnSC) device developed by McCluskey et al. [7, 23], a device designed specifically for calorimetry measurement on nanoscale materials in a high-throughput methodology. Compared to the Xensor device, the PnSC has a much smaller thermal impedance between heater, thermometer, and sample, resulting in a 
much faster temperature response and much higher accessible AC frequencies. These attributes enable adiabatic operation of the device in a high heat loss environment over a broad range of scan rates.

The main text of this chapter is organized into four sections. In Section 2.2, we present an in-depth analysis of scanning AC calorimetry measurement. In Section 2.3, we introduce the PnSC device used for the measurement and address issues related to temperature uniformity across the sample region. In Section 2.4, we describe the experimental set-up and discuss selection of the experimental parameters. In Section 2.5, we present experimental results for measurements on Sn thin-film samples for a range of frequencies and scan rates.

\subsection{Scanning AC calorimetry}

The application of AC calorimetry to measurements, in which the temperature is slowly ramped up, has been used in the past to investigate bulk materials [20, 33-35]. Here we apply the technique to nanocalorimetry. Because of the specific geometry of the nanocalorimetric sensor and the fast scanning rates, the conditions under which valid measurements can be performed need to be determined. Hence we briefly review the theory of AC calorimetry and adapt it to the specifics of the nanocalorimetry sensor used in the PnSC device. 
Consider a nanocalorimetry sensor that consists of a heating element with a temperature-dependent resistance. A sample of interest is placed on the sensor and is heated by sending a current through the heating element. The temperature of the sensor is determined by monitoring the resistance of the heater using a four-point measurement scheme (Figure 2.1). In the analysis, we assume that the temperature is uniform across the heating element and that the temperature difference between the heating element and the sample is negligible. The first assumption will be analyzed in more detail in the next section. The second assumption is valid as long as the thermal diffusion length is much larger than the sensor thickness at the time scale of the measurements and this is indeed the case for the sensors under consideration [7].

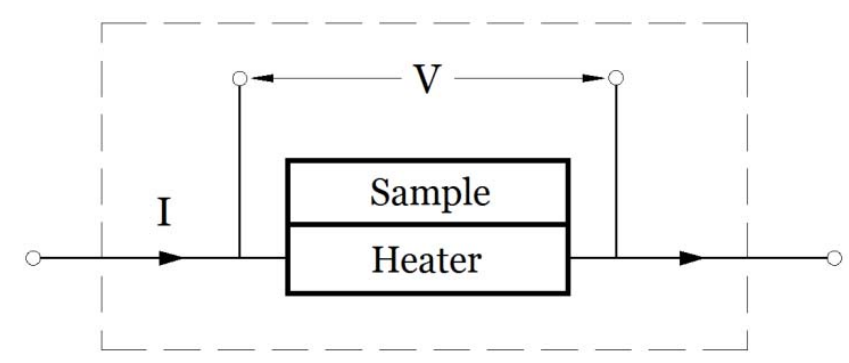

Figure 2.1. Schematic of a four-point measurement on a heating element with sample

In a typical AC nanocalorimetry experiment, a current with both direct current (DC) and $\mathrm{AC}$ components

$$
I=I_{0}+i \cos \left(\omega t-\varphi_{0}\right)
$$

is passed through the resistive heater. The AC component causes the temperature of the 
heater to oscillate around an average temperature $T_{0}$, which increases (in heating) or decreases (in cooling) with time. Hence the temperature response of the heater can be described as

$$
T=T_{0}(t)+\theta(t),
$$

where $T_{0}(t)$ is the average temperature over one oscillation period and $\theta(t)$ represents the oscillating part of the temperature. The amplitude of the oscillation is generally much smaller than the overall rise in temperature of the heater during the experiment. The temperature oscillations cause the resistance of the heating element $R$ to vary as [36]

$$
R=R_{0}(1+\lambda \theta),
$$

where $R_{0}$ and $\lambda$ are the resistance and the thermal coefficient of resistance (TCR) of the heating element at $T_{0}$, respectively. The power dissipated by the heater is given by Joule's law,

$$
\begin{aligned}
& P=I^{2} R=\left[I_{0}+i \cos \left(\omega t-\varphi_{0}\right)\right]^{2} R_{0}(1+\lambda \theta) \\
& =\left(I_{0}^{2}+\frac{1}{2} i^{2}\right) R_{0}(1+\lambda \theta)+2 I_{0} i R_{0}(1+\lambda \theta) \cos \left(\omega t-\varphi_{0}\right)+\frac{1}{2} i^{2} R_{0}(1+\lambda \theta) \cos 2\left(\omega t-\varphi_{0}\right)
\end{aligned}
$$

If $L(T)$ is the rate of heat loss from the heater and sample, then to first order

$$
L(T)=L_{0}\left(T_{0}\right)+\alpha \theta,
$$

where $L_{0}\left(T_{0}\right)$ and $\alpha$ are, respectively, the rate of heat loss at $T_{0}$ and its temperature 
derivative. The heat balance takes the form,

$$
P=C \dot{T}+L(T)=C\left(\dot{T}_{0}+\dot{\theta}\right)+L_{0}+\alpha \theta
$$

where $C$ is the total heat capacity of the heating element and the sample. Combining Eqs.

(2.4) and (2.6) leads to a first-order differential equation in the temperature:

$$
\begin{aligned}
& \left(I_{0}^{2}+\frac{1}{2} i^{2}\right) R_{0}(1+\lambda \theta)+2 I_{0} i R_{0}(1+\lambda \theta) \cos \left(\omega t-\varphi_{0}\right)+\frac{1}{2} i^{2} R_{0}(1+\lambda \theta) \cos 2\left(\omega t-\varphi_{0}\right) \\
& =C\left(\dot{T}_{0}+\dot{\theta}\right)+L_{0}+\alpha \theta
\end{aligned}
$$

To ensure that $R_{0}$ remains constant on the time scale of the oscillations, we take

$$
\lambda \dot{T}_{0} \frac{2 \pi}{\omega}<<1
$$

Furthermore realizing that

$$
\lambda \theta<<1,
$$

because both $\lambda$ and $\theta$ are small, we can reduce Eq. (2.7) to

$$
\left(I_{0}^{2}+\frac{1}{2} i^{2}\right) R_{0}+2 I_{0} i R_{0} \cos \left(\omega t-\varphi_{0}\right)+\frac{1}{2} i^{2} R_{0} \cos 2\left(\omega t-\varphi_{0}\right)=C\left(\dot{T}_{0}+\dot{\theta}\right)+L_{0}+\alpha \theta
$$

If we take the time average of Eq. (2.10) over one oscillation period, all oscillating terms vanish and we obtain the following simple equation in $T_{0}$

$$
\left(I_{0}^{2}+\frac{1}{2} i^{2}\right) R_{0}=C \dot{T}_{0}+L_{0}
$$

Equation (2.11) provides the relationship between the DC component of the applied power, the heat loss, and the ramp rate for the average temperature $T_{0}$. Subtracting Eq. (2.11) from Eq. (2.10) leads to a first-order ordinary differential equation in $\theta$ 


$$
2 I_{0} i R_{0} \cos \left(\omega t-\varphi_{0}\right)+\frac{1}{2} i^{2} R_{0} \cos 2\left(\omega t-\varphi_{0}\right)=C \dot{\theta}+\alpha \theta .
$$

This equation allows calculation of the oscillating component of the temperature. The temperature $T_{0}$ enters Eq. (2.12) only via the temperature-dependence of $R_{0}$, which can be regarded as constant on the time scale of the oscillations by Eq. (2.8). The steady-state solution to Eq. (2.12) is given by

$$
\begin{aligned}
& \theta=\theta_{1} \cos \left(\omega t-\varphi_{0}-\varphi_{1}\right)+\theta_{2} \cos \left(2 \omega t-2 \varphi_{0}-\varphi_{2}\right) \\
& \theta_{1}=\frac{2 i I_{0} R_{0} \sin \varphi_{1}}{C \omega} \\
& \theta_{2}=\frac{i^{2} R_{0} \sin \varphi_{2}}{4 C \omega} \\
& \tan \varphi_{1}=\frac{C \omega}{\alpha} \\
& \tan \varphi_{2}=\frac{2 C \omega}{\alpha}
\end{aligned}
$$

While the applied current has an angular frequency $\omega$ only, the temperature oscillation has both $\omega$ and $2 \omega$ components because the applied power scales with the square of the current. Note that the amplitudes of both components scale inversely with the heat capacity $C$, and that the phases of the temperature oscillations with respect to the applied AC current are increasing functions of the ratio $C \omega / \alpha$. If $C \omega$ is much larger than $\alpha$, which is true under a broad range of experimental conditions, the phase angles $\varphi_{1}$ and $\varphi_{2}$ approach a value of

$$
\varphi_{1} \approx \varphi_{2} \approx \frac{\pi}{2} \text {. }
$$

This significantly simplifies the formulas and hence the data analysis. Substituting the 
expression for $\theta$ given by Eq. (2.13a) into Eq. (2.3) yields an expression for the resistance of the heating element

$$
R=R_{0}\left[1+\lambda \theta_{1} \cos \left(\omega t-\varphi_{0}-\varphi_{1}\right)+\lambda \theta_{2} \cos \left(2 \omega t-2 \varphi_{0}-\varphi_{2}\right)\right]
$$

The voltage drop $V$ across the heating element is then given by Ohm's law,

$$
V=\left[I_{0}+i \cos \left(\omega t-\varphi_{0}\right)\right] R_{0}\left[1+\lambda \theta_{1} \cos \left(\omega t-\varphi_{0}-\varphi_{1}\right)+\lambda \theta_{2} \cos \left(2 \omega t-2 \varphi_{0}-\varphi_{2}\right)\right]
$$

In addition to a DC component, the voltage response has $\omega, 2 \omega$ and $3 \omega$ harmonic components. It is useful to deconvolve the voltage response into the four frequency components, $V_{0}, V_{\omega}, V_{2} \omega$, and $V_{3 \omega}$, whose sum is equal to the full voltage response (Eq.

2.16). The $D C$ component is given by

$$
V_{0}=I_{0} R_{0}+\frac{1}{2} i R_{0} \lambda \theta_{1} \cos \varphi_{1} \approx I_{0} R_{0}
$$

while the harmonic components are

$$
\begin{aligned}
& V_{\omega}=X_{\omega} \cos \omega t+Y_{\omega} \sin \omega t \\
& X_{\omega}=i R_{0} \cos \varphi_{0}+I_{0} R_{0} \lambda \theta_{1} \cos \left(\varphi_{0}+\varphi_{1}\right)+\frac{1}{2} i R_{0} \lambda \theta_{2} \cos \left(\varphi_{0}+\varphi_{2}\right), \\
& Y_{\omega}=i R_{0} \sin \varphi_{0}+I_{0} R_{0} \lambda \theta_{1} \sin \left(\varphi_{0}+\varphi_{1}\right)+\frac{1}{2} i R_{0} \lambda \theta_{2} \sin \left(\varphi_{0}+\varphi_{2}\right), \\
& V_{2 \omega}=X_{2 \omega} \cos (2 \omega t)+Y_{2 \omega} \sin (2 \omega t) \\
& X_{2 \omega}=\frac{1}{2} i R_{0} \lambda \theta_{1} \cos \left(2 \varphi_{0}+\varphi_{1}\right)+I_{0} R_{0} \lambda \theta_{2} \cos \left(2 \varphi_{0}+\varphi_{2}\right), \\
& Y_{2 \omega}=\frac{1}{2} i R_{0} \lambda \theta_{1} \sin \left(2 \varphi_{0}+\varphi_{1}\right)+I_{0} R_{0} \lambda \theta_{2} \sin \left(2 \varphi_{0}+\varphi_{2}\right), \\
& V_{3 \omega}=X_{3 \omega} \cos (3 \omega t)+Y_{3 \omega} \sin (3 \omega t), \\
& X_{3 \omega}=\frac{1}{2} i R_{0} \lambda \theta_{2} \cos \left(3 \varphi_{0}+\varphi_{2}\right),
\end{aligned}
$$




$$
Y_{3 \omega}=\frac{1}{2} i R_{0} \lambda \theta_{2} \sin \left(3 \varphi_{0}+\varphi_{2}\right)
$$

In practice, the resistance of the heating element is readily calibrated to temperature, so that $T_{0}$ can be determined directly from Eq. (2.17). According to Eq. (2.9), the terms containing $\vartheta_{1}$ and $\vartheta_{2}$ in Eq. (2.18) are much smaller than terms in $i R_{0}$. Thus Eq. (2.18) cannot generally provide accurate information on the heat capacity. It is possible, however, to determine the heat capacity from either Eq. (2.19) or (2.20). Substituting Eqs. (2.13) and (2.14) into Eq. (2.19) and (2.20) finally yields

$$
\begin{aligned}
& X_{2 \omega}=-\left(\frac{1}{2} i \theta_{1}+I_{0} \theta_{2}\right) R_{0} \lambda \sin \left(2 \varphi_{0}\right)=-\frac{5 i^{2} I_{0} R_{0}^{2} \lambda}{4 C \omega} \sin \left(2 \varphi_{0}\right), \\
& Y_{2 \omega}=\left(\frac{1}{2} i \theta_{1}+I_{0} \theta_{2}\right) R_{0} \lambda \cos \left(2 \varphi_{0}\right)=\frac{5 i^{2} I_{0} R_{0}^{2} \lambda}{4 C \omega} \cos \left(2 \varphi_{0}\right), \\
& X_{3 \omega}=-\frac{1}{2} i R_{0} \lambda \theta_{2} \sin \left(3 \varphi_{0}\right)=-\frac{i^{3} R_{0}^{2} \lambda}{8 C \omega} \sin \left(3 \varphi_{0}\right) \\
& Y_{3 \omega}=\frac{1}{2} i R_{0} \lambda \theta_{2} \cos \left(3 \varphi_{0}\right)=\frac{i^{3} R_{0}^{2} \lambda}{8 C \omega} \cos \left(3 \varphi_{0}\right) .
\end{aligned}
$$

In the adiabatic limit, the heat capacity $C$ can be calculated from the amplitude of the $2 \omega$ signal

$$
C=\frac{5 I_{0} i^{2} R_{0}^{2} \lambda}{4 \omega \sqrt{X_{2 \omega}^{2}+Y_{2 \omega}^{2}}}=\frac{5 I_{0} i^{2} R_{0}^{2} \lambda}{4 \omega\left|V_{2 \omega}\right|},
$$

or the $3 \omega$ signal

$$
C=\frac{i^{3} R_{0}^{2} \lambda}{8 \omega \sqrt{X_{3 \omega}^{2}+Y_{3 \omega}^{2}}}=\frac{i^{3} R_{0}^{2} \lambda}{8 \omega\left|V_{3 \omega}\right|}
$$


Equation (2.24) reproduces the expression for the heat capacity in the $3 \omega$ method used previously for isothermal calorimetry measurements [34]. The $2 \omega$ component in the signal and consequently Eq. (2.23) arise because of the addition of a DC component to the applied current. This DC component provides increased flexibility in choosing the AC frequency and the amplitude of the temperature oscillation. The analysis presented in this section is based on three assumptions described by Eqs. (2.8), (2.9) and (2.14). These equations represent the conditions under which a scanning AC calorimetry measurement can be analyzed using either Eq. (2.23) or (2.24). When setting up a measurement, experimental parameters such as the $D C$ and $A C$ currents and the $A C$ frequency need to be selected to satisfy these conditions - a relatively straightforward exercise, as we will show in a later section of this paper.

\subsection{Nanocalorimetry device}

\section{A. Description of the device}

The nanocalorimetry measurements in this study were performed using a parallel nano-scanning calorimeter (PnSC). The PnSC is a thin-film device described in detail previously [7, 23]. Briefly, it consists of silicon substrate with a $5 \times 5$ array of independently controlled micromachined calorimeter cells. Each cell contains a tungsten four-point electrical probe that serves both as a heating element and as a resistance 
thermometer. The tungsten probe is supported by a freestanding silicon nitride membrane and is completely encapsulated in silicon nitride. Figure $2.2 \mathrm{a}$ shows a schematic of the cell used for scanning calorimetry measurements in prior work [7], with a thin-film sample deposited in the shaded area between the two sensing leads.

In a typical scanning nanocalorimetry experiment, an electric current is supplied through the tungsten heating element, heating the sample at a rate of approximately $1-3 \times 10^{4} \mathrm{~K} / \mathrm{s}$ in vacuum [7]. The fast heating rate mitigates heat loss to the environment and results in a uniform temperature distribution across the sample region [7]. At slower heating rates $\left(<10^{3} \mathrm{~K} / \mathrm{s}\right)$, however, a large fraction of input power is lost as heat, yielding conditions that are more appropriate for AC calorimetry. In the "strip" heating element design of Figure 2.2a the heat loss has an additional deleterious effect of yielding poor temperature uniformity over the sample region. We enable calorimetry measurements in this heat rate regime by not only employing $A C$ calorimetry, but also incorporating a new device design that provides the necessary temperature uniformity.

This new 'serpentine' device is depicted in Figure 2.2b. The length of the heating element is increased by wrapping the heating strip into a 3-fold serpentine. The sample is located on the center strip between the sensing probes. The outer two legs of the heater serve to minimize conductive heat loss to the Si substrate, thus improving the temperature uniformity across the sample region. Figure 2.3 shows the full metallization 
layout of the PnSC device with an array of 25 of these four-point serpentine sensors.

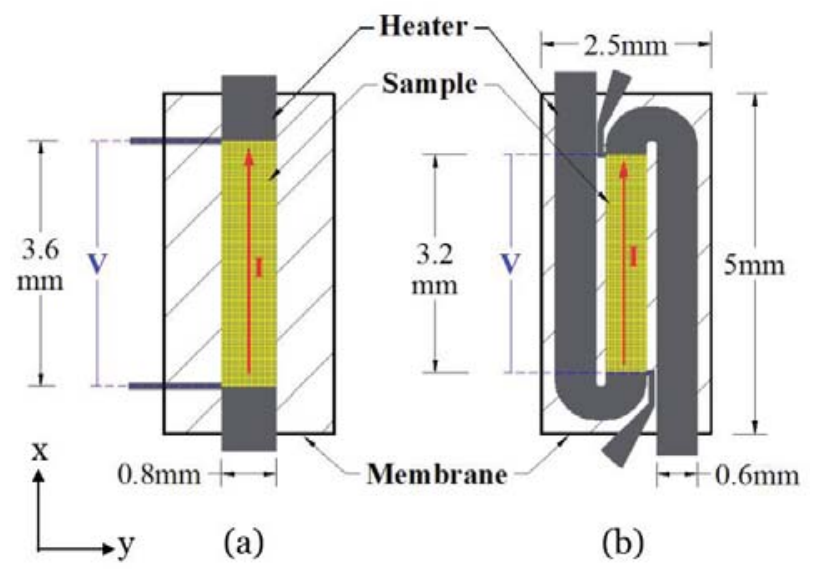

Figure 2.2. Layout of the calorimeter cell: strip (a) and serpentine (b)

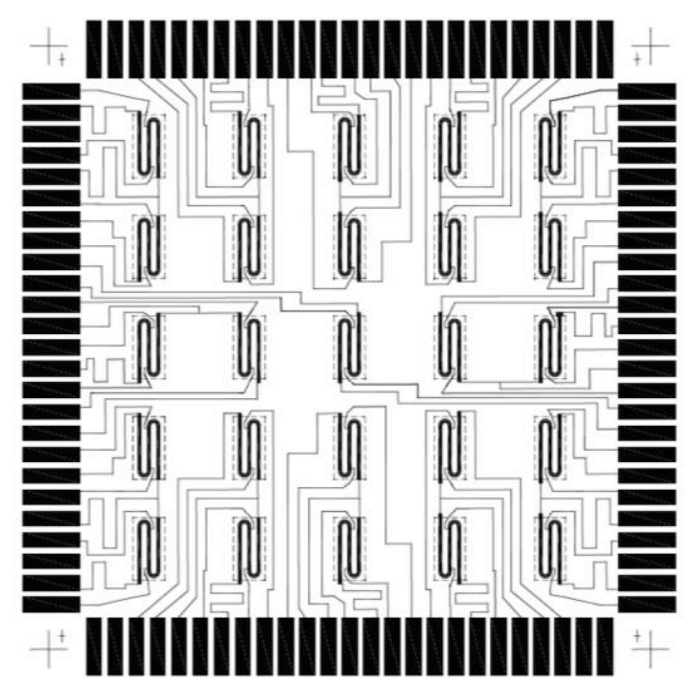

Figure 2.3. Metallization layout of the serpentine PnSC device. The lead lines are schematic with reduced width for clarity.

\section{B. Thermal modeling of the calorimetry sensor}

To quantify the in-plane temperature distribution in the sample area of the nanocalorimetry sensor, we have constructed finite element models for both the strip and the serpentine designs using the commercial software COMSOL Multiphysics 4.1a. 
In the $x-y$ plane, the models have the same dimensions as the sensors shown in Figure 2.2. The models contain a common tungsten thermistor thickness of $125 \mathrm{~nm}$ and, where applicable, a tin sample with a thickness of $100 \mathrm{~nm}$. Based on calibrations of actual PnSC sensors, the electrical resistivity of the tungsten heater was modeled as a linearly increasing function of temperature, with a room temperature value of $1.58 \times 10^{-7} \Omega \mathrm{m}$, three times its bulk value, and a thermal coefficient of resistance of $0.00165 \mathrm{~K}^{-1}$ [7]. The increase in resistivity above the bulk value is caused by the extremely fine microstructure of the sputtered tungsten. The thermo-physical parameters used in the models are provided in Table 2.1 and the temperature dependence of these parameters was neglected. The thermal conductivities used in the simulations were one third of their bulk values in accordance with the Wiedemann-Franz law. In a typical experiment, the thermal diffusive length scale is much larger than the physical length scale in the out-of-plane direction, resulting in less than $0.1 \mathrm{~K}$ of temperature variation through the thickness of the membrane and the heating element $[7,23]$. The small out-of-plane thermal gradient allowed us to use a more computationally efficient 2-D equivalent model of the device.

The heating process was simulated for sensors with and without the $100 \mathrm{~nm}$ tin sample. During the simulation, the boundary of the cell, where the silicon nitride membrane was supported by the silicon wafer, was held at ambient temperature $\left(20^{\circ} \mathrm{C}\right)$ 
because the silicon substrate has a much larger thermal mass than the sensor and it serves as a perfect sink for any heat conducted through the membrane. Both steady-state and time-dependent simulations were conducted. For the steady-state simulations, a constant current was applied to the heater and the corresponding steady-state temperature distribution was determined. By repeating the simulation with currents ranging from low to high, we obtained a series of steady-state temperature distributions with increasing average temperature across the sensor. For the time-dependent simulations, a linearly increasing current was applied to the heating element and the temperature distribution was determined as a function of time. The heating rate was calculated as the time derivative of the average temperature across the sample region. To simulate vacuum conditions, only radiative heat loss with an emissivity $\varepsilon_{c}$ of 0.104 [23] was allowed from the top and bottom surfaces of the sensor. We also performed several steady-state simulations in a He environment, where heat loss occurred by conduction through the He gas in addition to radiation. The conductivity of He was taken to be $0.15 \mathrm{Wm}^{-1} \mathrm{~K}^{-1}$ [37]. Convective heat loss was neglected because of the very small Raleigh number.

Typical steady-state simulation results for strip and serpentine devices with a Sn sample are shown in Figure 2.4. The temperature distribution in the sample area is clearly much more uniform for the serpentine device than for the strip device in both 
vacuum and helium environments. The steepest temperature gradients now occur in the outer legs of the serpentine, leaving the center leg and thus the sample region more uniform. The presence of the He improves the temperature distribution in the longitudinal direction, but makes it slightly worse in the transverse direction because of enhanced heat loss from the silicon nitride membrane. To compare the temperature uniformity of different experiments and at different temperatures, we define the relative temperature uniformity, $\gamma$, of the sensor as

$$
\gamma=\frac{1}{T_{0}-T_{R T}} \frac{1}{S} \int\left|T_{0}-T\right| d S^{\prime},
$$

Table 2.1. Parameters used in the FEM models [38-40]

\begin{tabular}{|c|c|c|c|c|c|c|}
\hline \multicolumn{2}{|c|}{} & $\begin{array}{c}\text { Length } \\
(\mathrm{mm})\end{array}$ & $\begin{array}{c}\text { Width } \\
(\mathrm{mm})\end{array}$ & $\begin{array}{c}\text { Thickness } \\
(\mathrm{nm})\end{array}$ & $\begin{array}{c}\text { Heat } \\
\text { Capacity } \\
(\mathrm{J} / \mathrm{kg} / \mathrm{K})\end{array}$ & $\begin{array}{c}\text { Thermal } \\
\text { conductivity } \\
(\mathrm{W} / \mathrm{m} / \mathrm{K})\end{array}$ \\
\hline \multirow{2}{*}{$\begin{array}{c}\text { Thermistor } \\
(\mathrm{W})\end{array}$} & Strip & 5 & 0.8 & 125 & 132 & 57.67 \\
\cline { 2 - 5 } & Serp. & 15.77 & 0.6 & 125 & & \\
\hline \multirow{2}{*}{$\begin{array}{c}\text { Membrane } \\
\left(\mathrm{SiNi} \mathrm{i}_{\mathrm{x}}\right)\end{array}$} & Strip & 5 & 2.5 & 125 & 700 & 3.2 \\
\cline { 2 - 5 } & Serp. & 5 & 2.5 & 125 & & \\
\hline $\begin{array}{c}\text { Sample } \\
(\mathrm{Sn})\end{array}$ & Strip & 3.6 & 0.8 & 100 & 222 & 22.27 \\
\cline { 2 - 5 } & Serp. & 3.2 & 0.6 & 100 & & \\
\hline
\end{tabular}




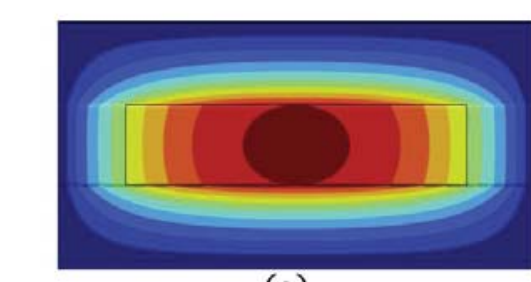

(a)

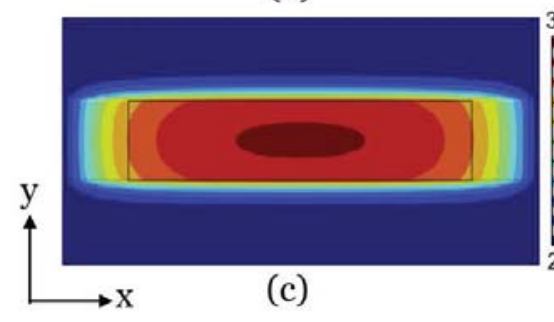

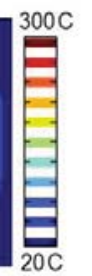

西

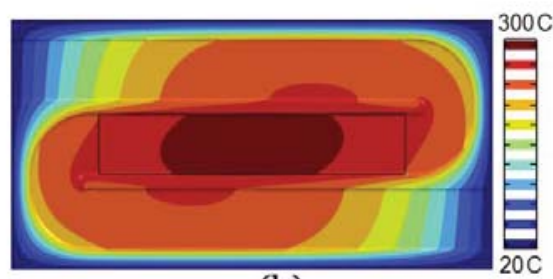

(b)

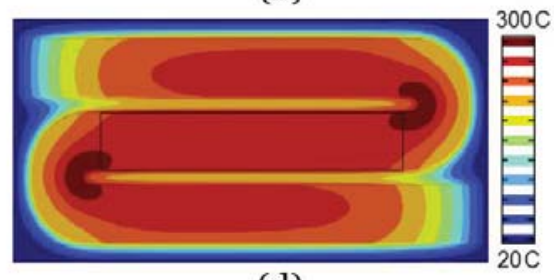

(d)

Figure 2.4. Typical simulation results for (a) the strip design in vacuum, (b) the serpentine design in vacuum, (c) the strip design in $\mathrm{He}$, and (d) the serpentine design in He. All simulations are for sensors in the steady state with a Sn sample in place. All contour lines are equally spaced. Applied currents are 46 $\mathrm{mA}, 26 \mathrm{~mA}, 65 \mathrm{~mA}$ and $63 \mathrm{~mA}$, respectively.

where $T_{R T}$ represents room temperature and $T_{0}$ is the average temperature across the sample area $S$. Figure $2.5 \mathrm{a}$ and $2.5 \mathrm{~b}$ show, as a function of average sample temperature, the temperature uniformity in vacuum for three different heating rates. The three heating rates are chosen to represent slow (quasi steady-state), medium $\left(1.5 \times 10^{3} \mathrm{~K} / \mathrm{s}\right)$ and fast $\left(1.5 \times 10^{4} \mathrm{~K} / \mathrm{s}\right)$ heating conditions. In most cases, the addition of the sample to the empty sensor improves temperature uniformity due to the enhancement in lateral thermal conduction, which suppresses thermal gradients. Only at very fast heating rates does the sample degrade the temperature uniformity of the serpentine device slightly. In vacuum, the strip design has good temperature uniformity $(\gamma<5 \%)$ for the fastest heating rate, in agreement with previous reports [7]. At slower heating rates, however, the temperature uniformity is significantly degraded mainly because of heat loss to the 
Si substrate via the tungsten heating element. These results are consistent with prior simulations of strip devices in the steady state [41]. The serpentine design, on the other hand, shows improved temperature uniformity over the entire temperature range and for all heating rates, with $\gamma$ around $3 \%$ at $200^{\circ} \mathrm{C}$ and decreasing to approximately $1 \%$ at $1000^{\circ} \mathrm{C}$. We note that the temperature uniformity of the strip design improves significantly as the average temperature increases, because the increased radiative heat loss provides negative feedback for temperature non-uniformity. A similar effect is seen with the He atmosphere (Figure 2.5c), with the strip design yielding good temperature uniformity $(<5 \%)$ in the steady state, which is a significant improvement compared to the in-vacuum condition. Since the serpentine device already yields good uniformity in the vacuum condition, the addition of He has a relatively small effect. 


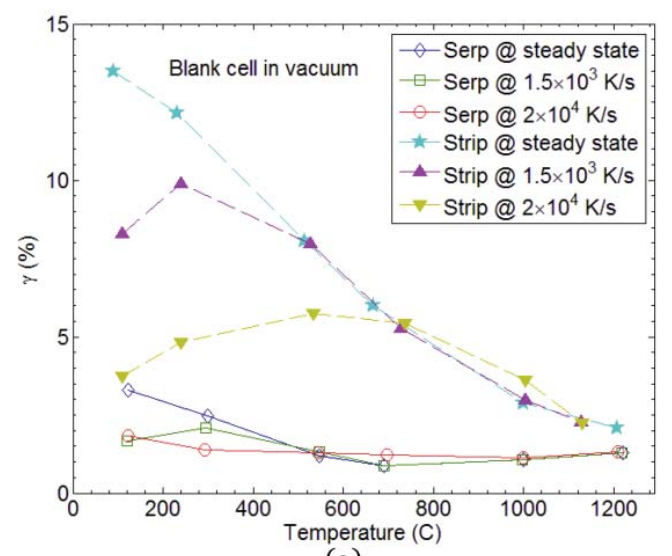

(a)

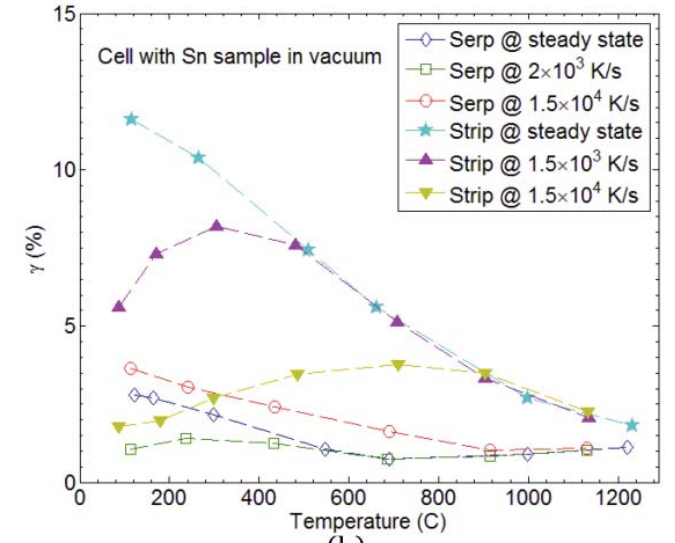

(b)

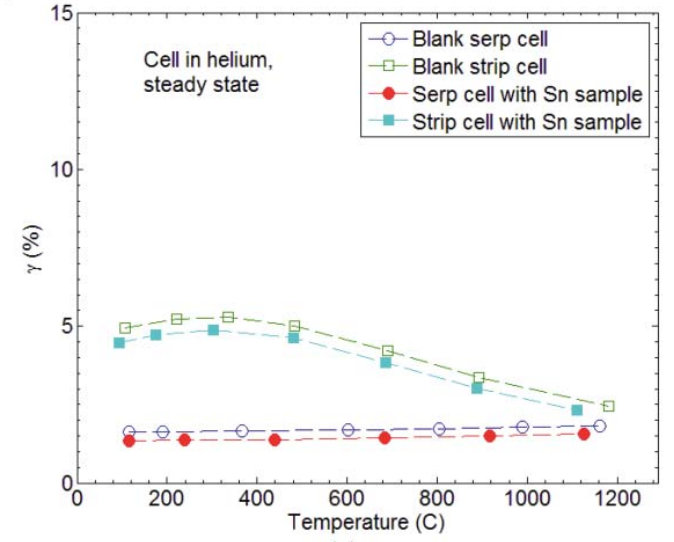

(c)

Figure 2.5. FEM simulation results: Temperature uniformity $\gamma$ for (a) blank cells in vacuum, (b) cells with $\mathrm{Sn}$ samples in vacuum, and (c) cells in the steady state in He. The heating rates at $300^{\circ} \mathrm{C}$ is used as the nominal heating rate.

\subsection{Experimental detail}

\section{A. Measurement setup}

Figure 2.6 shows a schematic of the measurement set up. The PnSC device is installed in a probe card that is mounted inside an ultra-high vacuum (UHV) furnace, with a base pressure of $1 \mathrm{e}-7$ Torr. The UHV furnace is pumped down by a turbo pump (Varian Turbo-V551 Navigator), which is backed by a mechanical pump (Varian TriScroll 
300). The furnace provides a chemically inert ambient for the PnSC sensor, enabling measurements in both vacuum and inert gas environments. A custom-designed measurement system serves as a programmable current source and data acquisition (DAQ) system for the PnSC sensors. The systems consists of several sub-systems as illustrated in Figure 2.6: (1) A current source with current source controller for powering the PnSC sensors and for monitoring the current output; (2) a DAQ controller for acquiring the voltage output signals from the PnSC sensors; (3) a multiplexer used to select individual sensors for calorimetry measurements by establishing the four electrical connections; (4) an amplifier board for conditioning of the sensor's voltage response and (5) a host interface for executing write/read command from the user computer communicated via an Ethernet cable. The multiplexer consists of a set of mechanical relays, which have negligible crosstalk and near infinite open-state resistance. The host interface communicates to the current source/DAQ controllers through a fiber optic loop and the experiment electronics are powered by low noise DC power supplies (Agilent E3630A and E3620A). The use of fiber optic communication provides galvanic isolation of the experiment circuit from the control electronics. The instrumentation power is also ground-isolated such that the entire experiment circuit is ground-isolated and ground-shielded. The integrated measurement system is controlled via a custom LabView ${ }^{\oplus}$ program on the user computer. 
The current source is a modified Howland voltage-to-current converter consisting of three high-precision, low noise operational amplifiers (OPA2227, Texas Instruments, Austin, TX) and a high-output current differential driver (AD815, Analog Devices, Norwood, MA). The current source has adjustable output ranges (25 mA, $50 \mathrm{~mA}, 125 \mathrm{~mA}$, $250 \mathrm{~mA}$, and $500 \mathrm{~mA}$ ) and an output compliance of $25 \mathrm{~V}$. The effective output resistance of the current source is significantly larger than $1 \mathrm{M} \Omega$, which is four orders of magnitude larger than the resistance of the sensors in a typical experiment. A simultaneous-sampling 16-bit per channel ADC/DAC board applies the current program and measures both the applied current (voltage across a precision resistor) and voltage response at a maximum rate of $300 \mathrm{kHz}$. Each $\mathrm{D} / \mathrm{A}$ and $\mathrm{A} / \mathrm{D}$ converter has a memory depth of $1 \mathrm{MB}$, and is calibrated using precision resistors (Vishay VPR221Z).

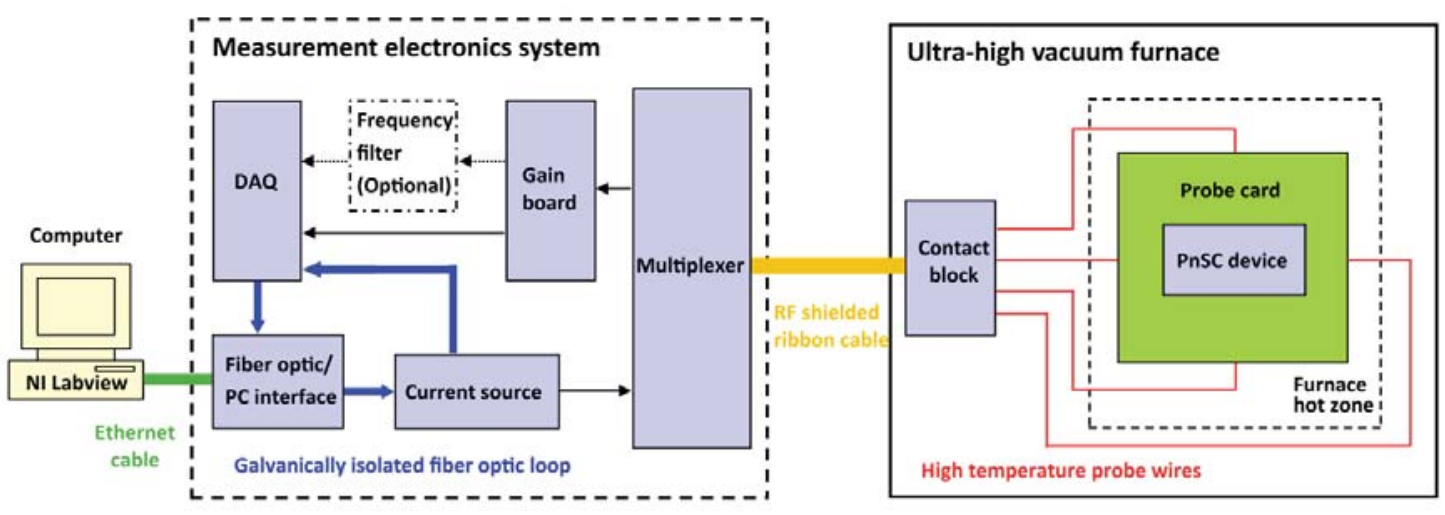

Figure 2.6. Schematic of experimental setup.

During a typical measurement, the host interface receives from the user computer a pre-defined digital waveform of input current versus time. A control voltage is sent from 
the current source controller to the current source, which then passes the analog current through the heating element on the PnSC sensor. The current source controller monitors the input current by measuring the voltage drop across a precision resistor (Vishay VPR221Z) in series with the heating element. The voltage output from the PnSC sensor is amplified and digitized by the DAQ controller. Current input and voltage output from the PnSC sensor are dynamically recorded, stored in digital format in DRAM on the host interface, and transferred to the user computer after completion of the measurement. Analysis of the data occurs in the digital domain, essentially converting the data acquisition system into a digital lock-in amplifier. Detailed circuitry schematics of the data acquisition system are provided in ref [22].

\section{B. Device fabrication, calibration, and sample preparation}

A PnSC device with serpentine design was fabricated using the same micromachining procedures as for the strip version, described in detail elsewhere [7]. Briefly, the fabrication process starts with a silicon nitride-coated (100) silicon wafer. Copper metallization and tungsten heating elements are sputter deposited and patterned on the front side of the wafer (Figure 2.3), and protected by a thin layer of silicon nitride deposited using plasma enhanced chemical vapor deposition. Rectangular windows are opened in the silicon nitride coating on the backside of the wafer using 
photolithography and reactive ion etching. The silicon is then etched in a concentrated aqueous $\mathrm{KOH}$ solution to make an array of freestanding silicon nitride membranes with tungsten heating elements.

After fabrication, the heating elements of the sensors in the PnSC device need to be calibrated to temperature. The resistance of the heating elements was measured by placing the PnSC device in a vacuum furnace and stepping the furnace temperature from room temperature to $170^{\circ} \mathrm{C}$ in approximately $15^{\circ} \mathrm{C}$ intervals. The vacuum furnace was filled with helium gas for better temperature uniformity within the chamber. At each temperature step, a DC current of $1 \mathrm{~mA}$ was applied to each heating element on the PnSC device and the voltage across the heating element was recorded. The resistance of the heating elements was found to increase linearly over the entire temperature range. The resistance, $R_{R T}$, and the thermal coefficient of resistance, $\lambda_{R T}$, at room temperature were determined from a linear least squares fit of the resistance data as a function of temperature,

$$
R=R_{R T}\left[1+\lambda_{R T}\left(T-T_{R T}\right)\right]
$$

Making the connection with $R_{0}$ and $\lambda$ at temperature $T_{0}$, we have

$$
\left.\frac{d R}{d T}\right|_{T_{0}}=R_{0} \lambda=R_{R T} \lambda_{R T}=k,
$$

where $k$ is defined as the temperature derivative of the resistance. Typical experimental values of $R_{R T}$ and $\lambda_{R T}$ were $5.37 \pm 0.02 \Omega$ and $1.65 \mathrm{e}-3 \pm 2 \mathrm{e}-5 \mathrm{~K}^{-1}$, respectively. 
After calibration of the heating elements, Sn, Bi and In samples were deposited onto the sensors by magnetron sputtering in a centered, on-axis geometry. The deposition was performed using a shadow mask to limit deposition to the center segment of each serpentine sensor. The deposition parameters are summarized in Table 2.2. A quartz crystal monitor was placed into the deposition geometry to measure the mass accumulation rate, from which the total deposited mass was calculated. The thickness values in Table 2.2 were calculated assuming standard density of the elemental solids. After deposition, the deposition mask was removed and an insulating aluminum nitride coating was deposited on all samples under substrate rotation to provide conformal coating. The $32 \mathrm{~nm}$-thick coating was deposited by $89 \mathrm{~W}$ of pulsed DC power in a $1.25 \mathrm{~Pa}$ atmosphere of $20 \% \mathrm{~N}_{2}$ in Ar. The refractory coating provided a protective barrier to oxidation during the calorimetry measurements.

Table 2.2. Sample deposition parameters

\begin{tabular}{|c|c|c|c|c|c|}
\hline material & Ar pressure(Pa) & Power $(\mathrm{W})$ & Rate $(\mathrm{nm} / \mathrm{s})$ & Thickness $(\mathrm{nm})$ & Mass $(\mu \mathrm{g})$ \\
\hline $\mathrm{In}$ & 1.3 & 11 & 0.25 & 190 & $2.6 \pm 0.4$ \\
\hline $\mathrm{Sn}$ & 2.4 & 6 & 0.14 & 100 & $1.4 \pm 0.2$ \\
\hline $\mathrm{Bi}$ & 0.67 & 15 & 1.1 & 300 & $5.6 \pm 0.5$ \\
\hline
\end{tabular}

\section{Experimental procedure and selection of AC measurement parameters}

First a series of DC scanning calorimetry measurements were conducted to establish the connection between applied current and heating/cooling rate. Measurements were 
performed in a 650 mTorr helium atmosphere. Because of the large thermal conductivity of helium and the small thermal mass of the nanocalorimetry sensor $(\sim 2 \mu \mathrm{J} / \mathrm{K})$, the sensors underwent a quasi steady-state temperature rise with a heating/cooling rate controlled directly by the ramp rate of the applied power. In our experiments, a constant current of $90 \mathrm{~mA}$ resulted in a steady-state sensor temperature of approximately $600^{\circ} \mathrm{C}$, and by applying an engineered current program, scanning rates ranging from 10 to $2 \times 10^{3}$ $\mathrm{K} / \mathrm{s}$ for both $\mathrm{DC}$ and $\mathrm{AC}$ measurements were achieved. By contrast, in a low-heat loss vacuum ambient, a constant current of $90 \mathrm{~mA}$ provides a heat rate of approximately $2 \times 10^{5} \mathrm{~K} / \mathrm{s}$ and a steady-state temperature in excess of $1100^{\circ} \mathrm{C}$.

Experimental parameters for AC measurements need to be selected to satisfy the conditions spelled out by Eqs. (2.8), (2.9) and (2.14). To better understand how these conditions can be met, we use the results in Eqs. (2.13) and (2.27) and substitute them into Eqs. (2.8), (2.9) and (2.14) to yield the following equivalent conditions:

$$
\begin{aligned}
& \lambda \theta \leq \frac{i k}{C \omega}\left(2 I_{0}+\frac{1}{4} i\right)=C_{1}<<1, \\
& \frac{k \dot{T}_{0}}{R_{0}} \frac{2 \pi}{\omega}=C_{2}<<1, \\
& \cot \varphi_{1}=\frac{\alpha}{C \omega}=C_{3}<<1 .
\end{aligned}
$$

In addition to these conditions, there are a two experimental considerations: 1 ) the $2 \omega$ voltage signal should be at least 20 times greater than the voltage corresponding to the least significant bit of the data acquisition system; 2 ) the frequency $\omega$ must be large 
enough that the mean temperature rise in a given cycle does not exceed the desired temperature resolution, typically about $2 \mathrm{~K}$ [17]. These considerations lead to the following additional conditions

$$
\frac{V_{2 \omega}}{\xi}=\frac{5 i^{2} I_{0} R_{0} k}{4 C \omega \xi}=C_{4} \geq 20,
$$

and

$$
\dot{T}_{0} \frac{2 \pi}{\omega}=C_{5} \leq 2 \mathrm{~K},
$$

where $\xi$ is the voltage corresponding to the least significant bit of the data acquisition system. While condition (28) limits the sub-cycle temperature oscillations, we do not state a condition that explicitly limits the instantaneous heating rate. Within a single cycle, the heating rate variations are proportional to $\omega \theta$ and for experiments that are sensitive to the instantaneous heating rate, an additional constraint should be added. Here we considered only the $2 \omega$ voltage because that signal is used in the experimental section. Equivalent conditions are easily derived for the $3 \omega$ signal. Table 2.3 lists typical values of experimental parameters in our experiments. The value of $\alpha$ was determined by estimating the heat loss from a calorimeter cell as a result of radiation and conduction through a helium atmosphere:

$$
\begin{aligned}
& L=\varepsilon_{c} \sigma\left(T^{4}-T_{R T}^{4}\right) S+\frac{K_{H e}}{D}\left(T-T_{R T}\right) S, \\
& \alpha=\frac{d L}{d T}=4 \varepsilon \sigma T^{3} S+\frac{K_{H e}}{D} S,
\end{aligned}
$$

where $\sigma$ is the Stefan-Boltzmann constant, $K_{H e}$ is the thermal conductivity of helium 
$\left(0.15 \mathrm{Wm}^{-1} \mathrm{~K}^{-1}[37]\right), S$ is the surface area of the calorimeter cell $\left(3.84 \times 10^{-6} \mathrm{~m}^{3}\right)$, and $D$ is the distance between cell to the wall of the chamber $\left(\sim 1 \times 10^{-2} \mathrm{~m}\right)$. The value of $\alpha$ increases with temperature and approaches $1.2 \times 10^{-4} \cdot \mathrm{W} / \mathrm{K}^{-1}$ at $600^{\circ} \mathrm{C}$.

It is possible to calculate $C$ and $\alpha$ from the $2 \omega$ voltage signal without resorting to the assumption that $\varphi_{1}$ and $\varphi_{2}$ approach the value $\pi / 2$ (Eqs. (2.14) and (2.30)). This approach requires solving non-linear equations and is not pursued here. These quantities can be computed in a mathematically simpler approach through Fourier analysis of the resistance $R$, which is readily determined by the ratio of the measured voltage and applied current. This method is, however, not well conditioned when $I_{0}$ and $i$ have similar magnitudes, because of the poor signal to noise ratio when the instantaneous applied current approaches zero.

Table 2.3. Typical experimental parameters

\begin{tabular}{|l|l|l|l|l|l|l|}
\hline$C(\mathrm{~J} / \mathrm{K})$ & $R_{R T}(\Omega)$ & $T_{0}(\mathrm{C})$ & $k(\Omega / \mathrm{K})$ & $\dot{T}_{0}(\mathrm{~K} / \mathrm{s})$ & $\xi(\mathrm{V})$ & $\alpha(\mathrm{W} / \mathrm{K})$ \\
\hline $2 \times 10^{-6}$ & 5.6 & $100-600$ & $9 \times 10^{-3}$ & $10^{1}-10^{3}$ & $10^{-5}$ & $1.2 \times 10^{-4}$ \\
\hline
\end{tabular}

The objective is to find the combination of $I_{0}, i$, and frequency $f=\omega / 2 \pi$ that best satisfies conditions (2.28) through (2.32). If the measurement is conducted in a He environment, the heating rate $\dot{T}_{0}$ is determined solely by the ramp rate of the input power and is independent of the instantaneous values of $I_{0}$ and $i$. Consequently, (2.29), (2.30), and (2.32) are independent requirements for the frequency, that reduce to the following two conditions, 


$$
\begin{aligned}
& f>>\frac{\alpha}{2 \pi C} \approx 9 \mathrm{~Hz}, \\
& f \geq \dot{T}_{0} \times 0.5 \mathrm{~K}^{-1} .
\end{aligned}
$$

The minimum frequency used in the experiments is $200 \mathrm{~Hz}$, which readily satisfies condition (2.35), and additionally satisfies condition (2.36) for the lowest heating rate of $10 \mathrm{k} / \mathrm{s}$. For the highest range of heating rates, condition (2.36) prescribes the use of higher frequencies.

Once the appropriate frequency has been selected, it is possible to determine allowable combinations of $I_{0}$ and $i$ from conditions (2.28) and (2.31). Condition (2.28) is independent of temperature, while condition (2.31) becomes somewhat less strict with increasing temperature. Figure 2.7 illustrates possible solutions for an AC frequency of $600 \mathrm{~Hz}$. Solid and dotted lines in the figure represent conditions at $80^{\circ} \mathrm{C}$ and $600^{\circ} \mathrm{C}$, respectively. The shaded area represents the locus of permissible combinations of $I_{0}$ and $i$ for typical experimental parameters (Table 2.3) at $T_{0}=80^{\circ} \mathrm{C}$. The two red lines are contours of constant average power. For measurements performed in He environments, they also represent equilibrium temperature isotherms. Connecting two points, one on each of the isotherms within the permissible region, with a straight line provides a locus of possible current combinations that leads to a temperature rise from 80 to $600^{\circ} \mathrm{C}$. A current program that follows this path through parameter space could provide a quasi-equilibrium sweep in temperature between the two extremes. Engineering of the 
heating rate is possible by not only varying the rate at which $i$ and $I_{0}$ are changed in the experiment, but also by choosing a path that remains in the permissible region and that maintains a monotonically increasing power. Conversely, a controlled-cooling rate experiment can be conducted by choosing a path inside the permissible region that maintains a monotonically decreasing power.

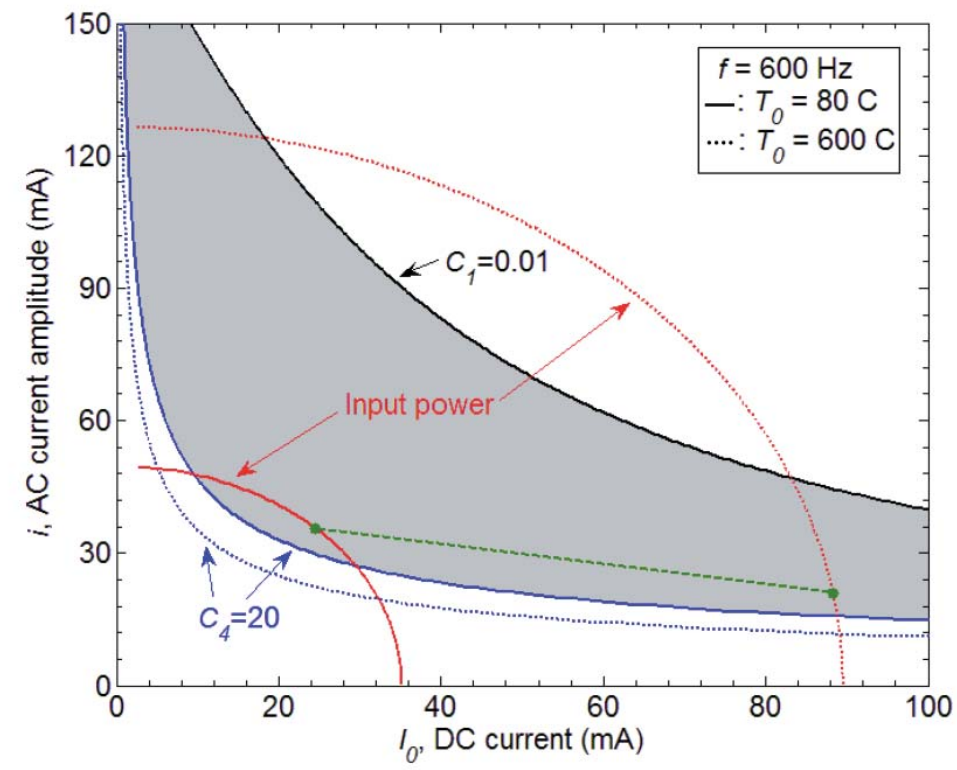

Figure 2.7. Geometric solution to inequality set (2.28) and (2.31), with experimentally determined input powers for the equilibrium temperature isotherms (red). The $C_{1}$ inequality is independent of temperature. The most restrictive contour of the $C_{4}$ inequality is used to define the permissible region (shaded area). The curves of constant input power correspond to the $80^{\circ} \mathrm{C}$ and $600^{\circ} \mathrm{C}$ isotherms.

\section{Data reduction}

To analyze the results, the measured currents and voltages for each experiment were divided into segments consisting of an integer number of AC oscillation periods. The signals $I_{0}, i$, and $V_{0}$ were obtained by performing a discrete Fourier transform (DFT) 
on every segment of the measured current and voltage, while the voltage. $V_{2 \omega}$ was evaluated by first passing the experimental voltage data through a digital band pass filter centered on $2 \omega$ and then applying a DFT to every segment. The band pass filter used in the algorithm was a $4^{\text {th }}$ order Butterworth band pass filter with cutoff frequencies $2 \omega \pm 150 \mathrm{~Hz}$. The filter removed the ramping DC component from the voltage data, thus preventing it from entering the windowed Fourier transform calculation and so distorting the $V_{2 \omega}$ signal. All discrete Fourier transform were performed using a second order Goertzel algorithm, which is a very efficient method of computing DFT values at specific frequencies [42]. The resistance $R_{0}$ was then calculated from $V_{0}$ and $I_{0}$ according to Eq. (2.17), and the temperature $T_{0}$ was obtained from Eq. (2.26). The total heat capacity $C$ of a cell was calculated using Eq. (2.23). Finally, a moving average method was implemented to reduce the noise in the heat capacity results [43].

\subsection{Results and discussion}

\section{A. Scanning $A C$ and DC calorimetry measurements}

Typical AC calorimetry signals from a PnSC sensor with and without a $100 \mathrm{~nm}$ Sn thin-film sample are shown in Figure 2.8a; Table 2.4 summarizes the results. The offset between the two signals is caused by the heat capacity of the sample. The difference between the signals normalized by the mass of sample is the specific heat capacity of 
the sample and is shown in Figure 2.8b. The experimental value of the specific heat capacity is $232 \pm 20 \mathrm{~J} / \mathrm{kg} \mathrm{K}$ at $100^{\circ} \mathrm{C}$ and slowly increases to $240 \pm 20 \mathrm{~J} / \mathrm{kg} \mathrm{K}$ at $550^{\circ} \mathrm{C}$. These results are in good agreement with the literature values for the heat capacity of bulk Sn [44], with the largest contribution to the error arising because of the uncertainty in the sample mass.

The heat capacity shows a peak near the melting temperature of $\mathrm{Sn}$. This peak is caused by melting of the Sn sample, but is not directly related to the latent heat of melting $[17,45]$. During the melting process, the AC signal can create oscillations in the relative fraction of the two phases (movement of the phase boundary), which leads to reduced temperature oscillations. This process is not accounted for in the derivation of Eq. (2.23) and results in a peak in the AC calculation of the heat capacity [46, 47]. This peak can be used to determine the melting temperature of the sample, but the area under the peak does not correspond to its latent heat of melting as it does in the typical DC analysis. Here, the melting temperature $\left(T_{m}\right)$ is taken at the maximum point of the heat capacity signal [7]. The value for $\mathrm{Sn}$ is found to be $230^{\circ} \mathrm{C}$, in good agreement with the literature value of $231.9^{\circ} \mathrm{C}$ [38]. The FWHM of the melting peak is determined mainly by the temperature uniformity of the sensor and the amplitude of the temperature oscillation (2-3K). This is in contrast with traditional calorimetry, where the width of the peak is governed by the heat transfer process between heater, sample, and 
thermometer, and where the temperature at the onset of the peak is taken as the melting temperature. In fact, in the PnSC, the sample thermally equilibrates with the heater in a time on the order of $10^{-5} \mathrm{~s}$, because of the nanometer-scale distance between the sample and the heating element and the intimate thermal contact between both [7]. At a heating rate of $10^{4} \mathrm{~K} / \mathrm{s}$, this results in a temperature difference of at most $0.1 \mathrm{~K}$ between sample and heating element.
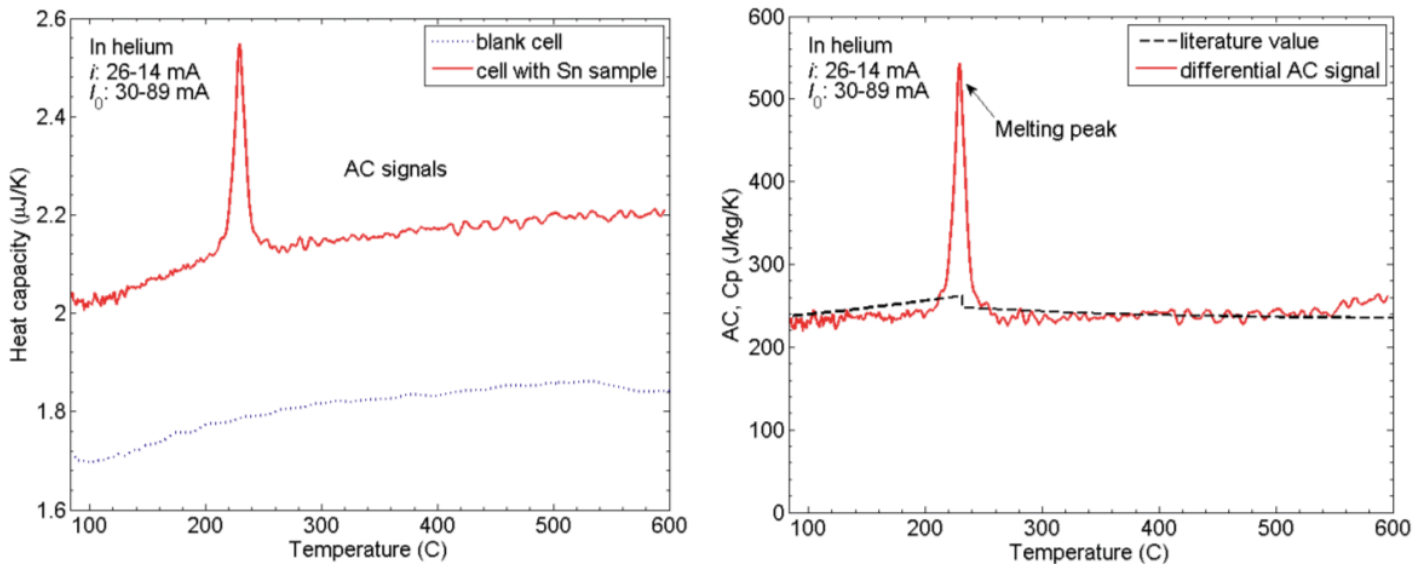

(a)

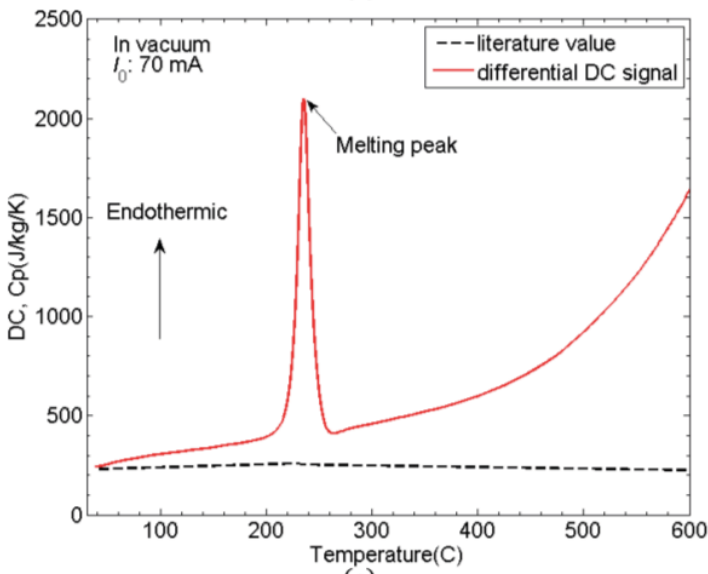

(c)

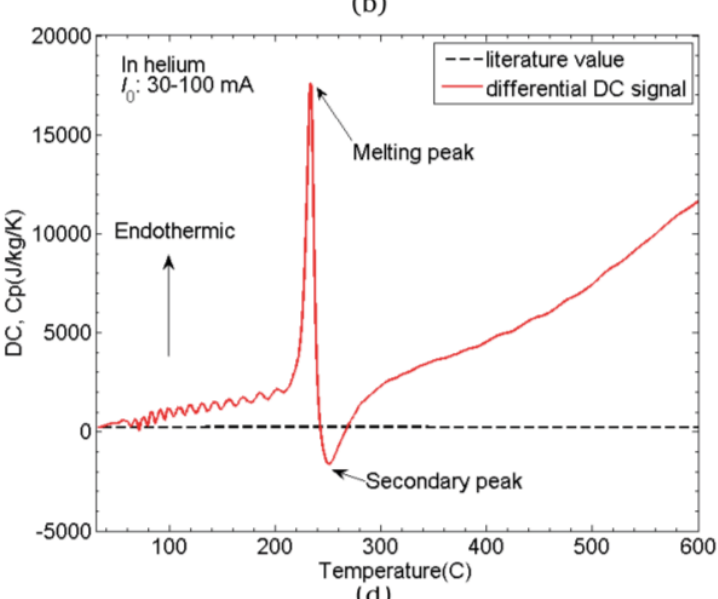

(d)

Figure 2.8. a) AC calorimetric signals obtained at a scanning rate of $10-10^{2} \mathrm{~K} / \mathrm{s}$ for sensors with and without a $100 \mathrm{~nm}$ Sn sample in a He environment using an AC frequency of $666.7 \mathrm{~Hz}$; b) Specific heat capacity of the Sn sample calculated from the AC measurement; c) Specific heat capacity of the Sn sample in vacuum measured at a heating rate of approximately $10^{4} \mathrm{~K} / \mathrm{s}$ using DC calorimetry; d) Specific heat capacity of the $\mathrm{Sn}$ sample in He measured at a heating rate of approximately $10^{3} \mathrm{~K} / \mathrm{s}$ using DC calorimetry. 
Table 2.4. Experimental results for Sn sample

\begin{tabular}{|c|c|c|}
\hline Methods & $T_{m}\left({ }^{\circ} \mathrm{C}\right)$ & FWMH $\left({ }^{\circ} \mathrm{C}\right)$ \\
\hline AC & 230 & 10.2 \\
\hline DC (fast) & 235 & 8.7 \\
\hline DC (slow) & 233 & 6.9 \\
\hline
\end{tabular}

To illustrate the effect of heat loss on the AC and DC methods, DC measurements were conducted on sensors with and without Sn sample. DC measurements performed in vacuum at a scanning rate of approximately $1.5 \times 10^{4} \mathrm{~K} / \mathrm{s}$ are representative of measurements taken under nearly adiabatic conditions; measurements performed in $\mathrm{He}$ at a scanning rate of $10^{3} \mathrm{~K} / \mathrm{s}$ are typical for measurements with significant heat loss to the environment. Results are summarized in Table 2.4. The specific heat capacities of the Sn sample obtained in these DC measurements are shown in Figure $2.8 \mathrm{c}$ and $2.8 \mathrm{~d}$; the corresponding heating rate curves are illustrated in Figure 2.9. Both for measurements performed in vacuum and in helium, the experimental values of the specific heat capacity agree well with the literature value near room temperature, but they rise quickly with increasing temperature as a result of heat loss to the environment. Part of this heat loss can be eliminated by making a differential measurement, i.e., by taking the difference between a sensor with and without sample, although this procedure is not sufficient to completely take out the effect of the heat loss, even for measurement in vacuum. Allen et al. have developed a more sophisticated technique to remove any heat loss in a DC measurement based on a direct differential measurement between two 
different sensors [48]. This method works very well if the samples represent a small fraction of the thermal mass of the sensor, which is not the case here. Comparison of the $A C$ (Figure 2.8b) and DC measurements (Figure 2.8c, 2.8d) clearly shows that the AC measurement is immune to heat loss, even though the average heating rate in the $A C$ measurement is two orders of magnitude smaller than for the slowest DC measurement (Figure 2.9).

The effect of heat loss on DC measurements is further illustrated by comparing Figs. 2.8c and $8 \mathrm{~d}$. In Figure $2.8 \mathrm{~d}$, the melting peak is followed by what appears to be an exothermic peak, denoted as secondary peak, which is absent in Figure 2.8c. The origin of the secondary peak is explained by considering the heat loss from the sample region during and right after melting. During melting, the average temperature of the sample region remains unchanged, while the temperature of the rest of the sensor increases. Consequently, the heat loss from the sample region to the rest of the sensor is slightly smaller at the end of melting process than at the start. Thus, the melting process first causes a dip in the heating rate of the sample, followed by a temporary increase right after melting (Figure 2.9). This increase in heating rate produces a secondary, mirrored peak in the heat capacity signal (Figure 2.8d). The secondary peak is noticeable whenever the heat loss from the sample region to the rest of the sensor is not negligible compared to the heating power, i.e., in measurements at relatively slow heating rates, 
and occurs for both endothermic and exothermic reactions. The secondary peak for an endothermic reaction is reflected as an additional exothermic peak in the heat capacity signal, and vice versa for an exothermic reaction. It should be regarded as an experimental artifact that arises because of the sensitivity of DC measurements to non-adiabatic conditions. Unlike DC calorimetry, AC measurements are not affected by heat loss from the sample. Consequently, they do not show a secondary peak in the heat capacity curve, as evident in Figs. 2.8a and 2.8b.

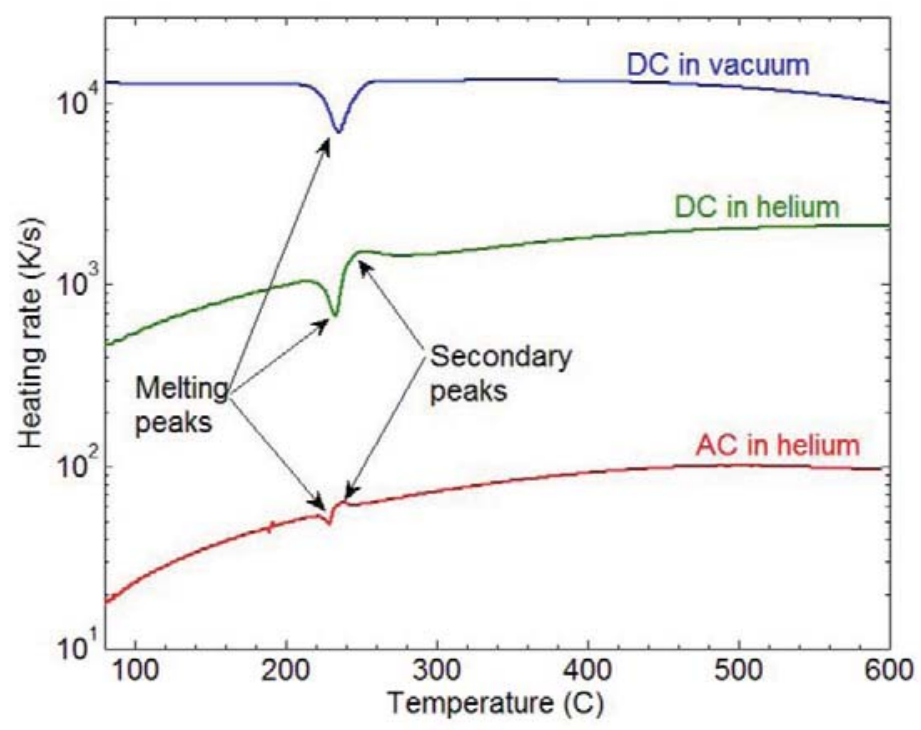

Figure 2.9. Heating rates for the AC and DC calorimetric measurements shown in Figure 2.8. The heating rate for the AC measurement refers to the cycle-averaged heating rate $\dot{\boldsymbol{T}}_{\mathbf{0}}$.

\section{B. The effect of frequency on the AC measurement}

When performing AC calorimetry measurements, it is instructive to explore the frequency dependence of the calorimetry results. AC calorimetry measurements can 
depend on the frequency at which the measurement is performed for a number of reasons. The thermal wave associated with the AC component of the power decays over a distance $\eta=\mathrm{V}\left(K_{m} / \omega \rho c\right)$, where $K_{m}$ and $c$ are the thermal conductivity and the specific heat capacity of the medium in which the wave propagates, respectively [31, 49]. Consequently, the volume of material - including sample and calorimeter - probed by the thermal wave scales with $\eta$. In other words, the volume of material for which the heat capacity is measured, changes inversely with the frequency [50]. Furthermore, the separation distance between the sample and thermometer results in the measured temperature oscillations being damped and delayed with respect to the temperature oscillations of the sample $[6,49]$. This effect is negligible if the separation is much smaller than $\eta$, but it increases rapidly as the separation exceeds $\eta$. As a result, the experimental value of the heat capacity increases with frequency if this effect is not properly accounted for in the analysis. Figs $2.10 \mathrm{a}$ and $2.10 \mathrm{~b}$ depict a series of calorimetric signals obtained at different AC frequencies for a sensor with a Sn sample and for a blank sensor, respectively. It is evident from the figures that the experimental values of the heat capacities are virtually identical, independent of measurement frequency, as are the heating rates. This is a direct result of the special geometry of the PnSC device: For typical frequencies, the separation distance between sample and heater/thermometer ( $100 \mathrm{~nm}$ ) in the PnSC device is at least an order of magnitude 
smaller than $\eta$ so that the measured temperature is an accurate reflection of the temperature of the sample. As the frequency decreases, the value of $\eta$ increases and sensor samples a larger volume of material, including the He in the ambient and the silicon nitride of the membrane. The results in Figs. 2.10a and 2.10b suggest that this effect is negligible. Indeed, the value of $\eta$ for silicon nitride is $10-30 \mu \mathrm{m}$, while that for $\mathrm{He}$ is $100-200 \mu \mathrm{m}$. The extra mass that lies within these distances from the heater is only a very small fraction of the total sampled mass and thus this frequency-dependent contribution to the heat capacity is negligible. We also note that this measurement artifact is easily removed by calculating the difference signal with the empty device, as was done in Figure 2.8b. These results demonstrate that the PnSC sensor is insensitive to the frequency of the $A C$ signal. We do observe that the height of the melting peak in the heat capacity signal increases with increasing frequency. As described above, this peak is not representative of the specific heat capacity and arises from the quenching of the temperature oscillations due to the coexistence of two phases. In principle the temperature oscillations could vanish during the melting process, providing a divergent heat capacity. A combination of non-equilibrium processes and the sensor's temperature non-uniformity may yield finite temperature oscillations; the windowed Fourier transform data reduction (see Sec. IIID) would require the temperature oscillations to vanish everywhere in the sensor over an entire window to be detected. We assert that 
the longer time averaging provided by a lower frequency reduces the peak value of the heat capacity signal.

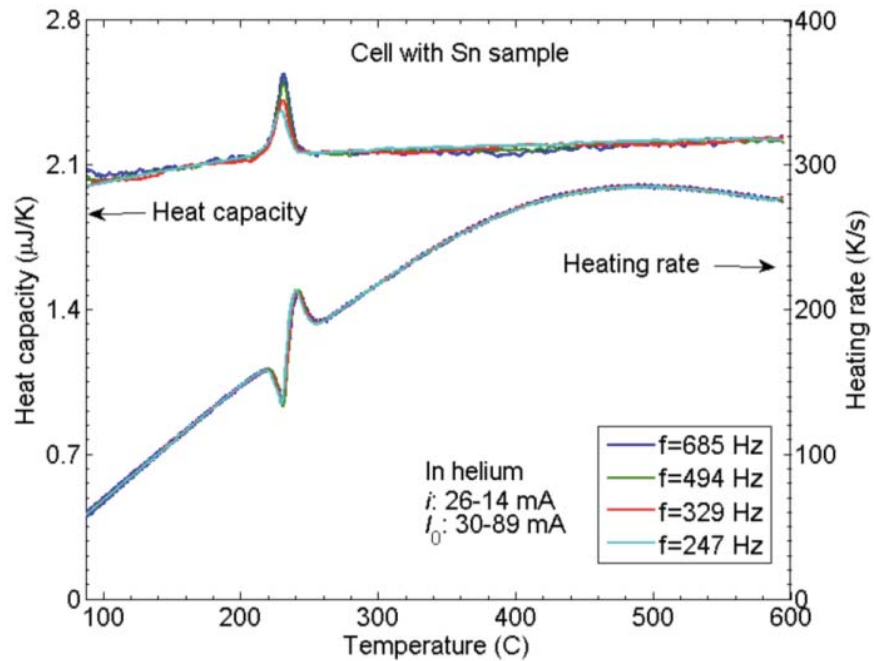

(a)

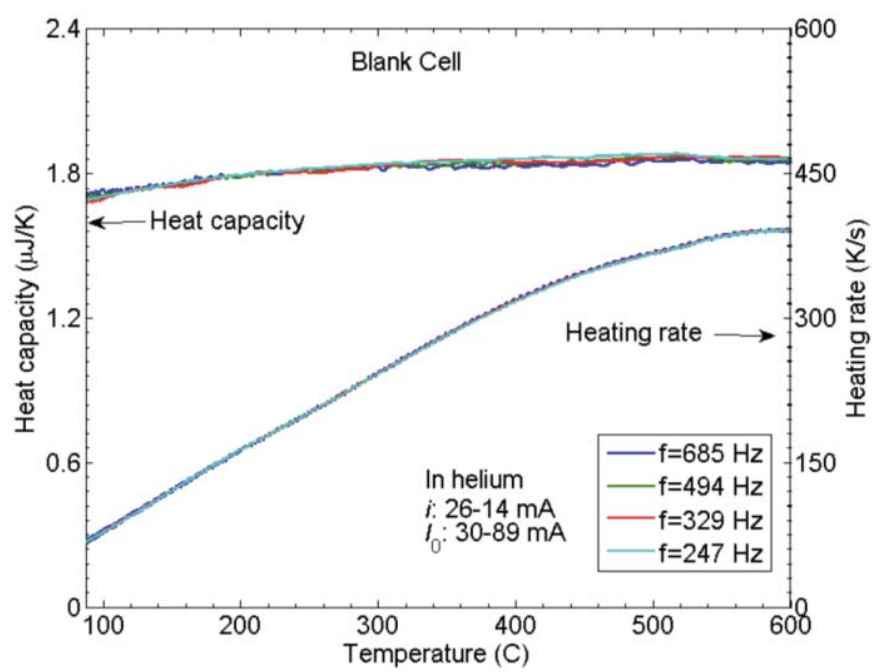

(b)

Figure 2.10. Comparison of AC calorimetric signals for different input frequencies: a) cell with a Sn sample;

b) blank cell. Input current increased linearly over a period of $3 \mathrm{~s}$. The heating rates refer to the cycle-averaged heating rate $\dot{T}_{\mathbf{0}}$.

\section{The effect of scanning rate on the AC measurements}

Figure 2.11 presents AC calorimetry results obtained at heating rates varying from 
10 to $2 \times 10^{3} \mathrm{~K} / \mathrm{s}$. The experimental values of the heat capacity are independent of the heating rate, with less than $1 \%$ difference between their average values. The constancy of the heat capacity demonstrates the capability of the $A C$ technique to perform measurements over a wide range of heating rates. There is a very small increase in melting temperature with increasing heating rate. In traditional calorimetry, such a shift is usually caused by the thermal impedances between heater, sample, and thermometer. In these measurements, these thermal impedances are very small and the temperature difference between sample and heating element is at most $0.1 \mathrm{~K}$. It is therefore more likely that the small shift in melting temperature is caused by a change in the temperature uniformity of the device with heating rate.

As mentioned in Section III, the heating rate for a measurement in He is controlled by how fast the input power increases. Conversely, by decreasing the input power from point $B$ to point $A$ as depicted in Figure 2.7 , it is possible to cool the sample from $600^{\circ} \mathrm{C}$ to $80^{\circ} \mathrm{C}$ at a controlled rate. Figure 2.12 shows the $A C$ results on heating and cooling for sensors with $100 \mathrm{~nm} \mathrm{Sn,} \mathrm{In} \mathrm{and} \mathrm{Bi} \mathrm{samples.} \mathrm{The} \mathrm{heat} \mathrm{capacities} \mathrm{measured} \mathrm{on} \mathrm{heating}$ and cooling are in very good agreement. The figure also shows distinct peaks for melting and solidification. In the AC measurements, solidification peaks appear as exothermic peaks, just like melting peaks, because the temperature oscillations are reduced during the solidification process. The result is an apparent increase in the value of $C p$ that is not 
directly related to the latent heat of the phase transformation. Whether a phase transformation is endothermic or exothermic is, of course, readily determined from the heating rate traces. Note that the peaks for In and Bi are broader than the peak for Sn because these measurements were performed using much larger AC oscillations than for the Sn sample. The measured melting points for $\mathrm{In}$ and $\mathrm{Bi}$ are $157.1^{\circ} \mathrm{C}$ and $275^{\circ} \mathrm{C}$, respectively. These experimental values are in good agreement with the literature values of $156.6 \mathrm{C}$ and $271.4^{\circ} \mathrm{C}$ [38]. On cooling, the solidification temperature for In coincides with its melting point, while Bi solidifies at $177.2^{\circ} \mathrm{C}$, well below its melting point. There is no sign of a Sn solidification peak over the measured temperature range. We attribute the undercooling of $\mathrm{Bi}$ and $\mathrm{Sn}$ to kinetically impeded nucleation of the solid phase. This explanation is in agreement with a recent observation that melted $\mathrm{Sn}$ on a graphite substrate can be undercooled as much as $56.1^{\circ} \mathrm{C}$ below its equilibrium melting temperature when cooled at a rate of $1 \mathrm{~K} / \mathrm{s}$ [51]. The results in Figure 2.12 suggest an undercooling for $\mathrm{Sn}$ of at least $132^{\circ} \mathrm{C}$ below its melting temperature, which is reasonable given that the cooling rate is two orders of magnitude larger than in the previous observation. 


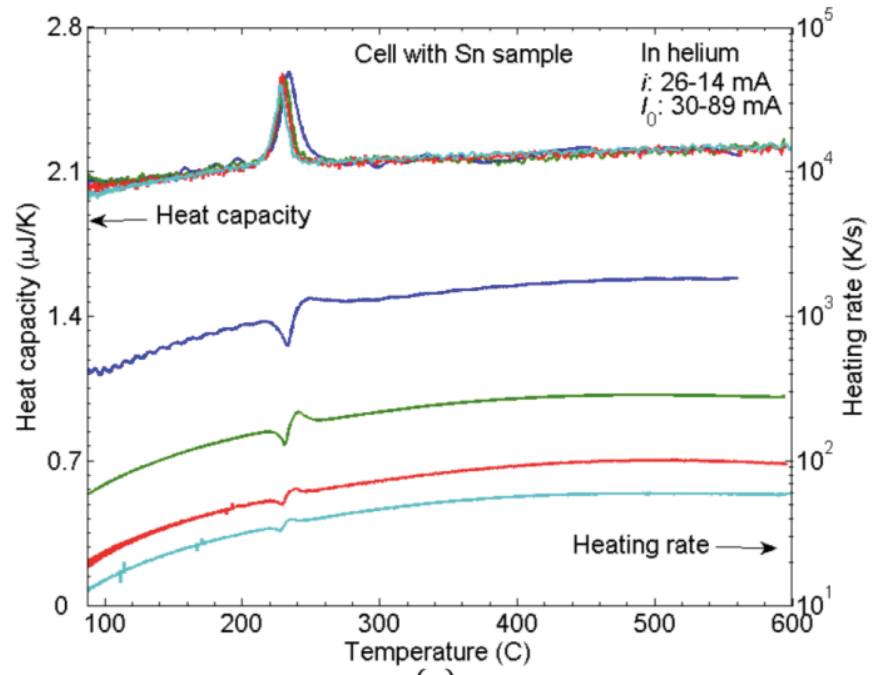

(a)

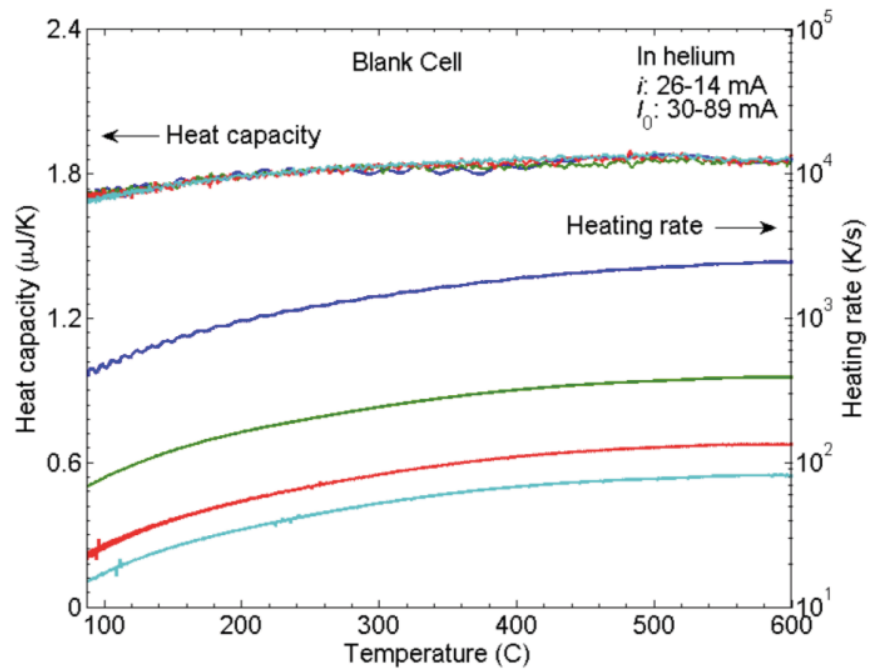

(b)

Figure 2.11. AC calorimetric signals for different heating rates: a) sensor with a Sn sample; b) blank sensor. Input current increased linearly over a period of $0.5 \mathrm{~s}, 3 \mathrm{~s}$, and $15 \mathrm{~s}$. The heating rates refer to the cycle-averaged heating rate $\dot{T}_{\mathbf{0}}$. 


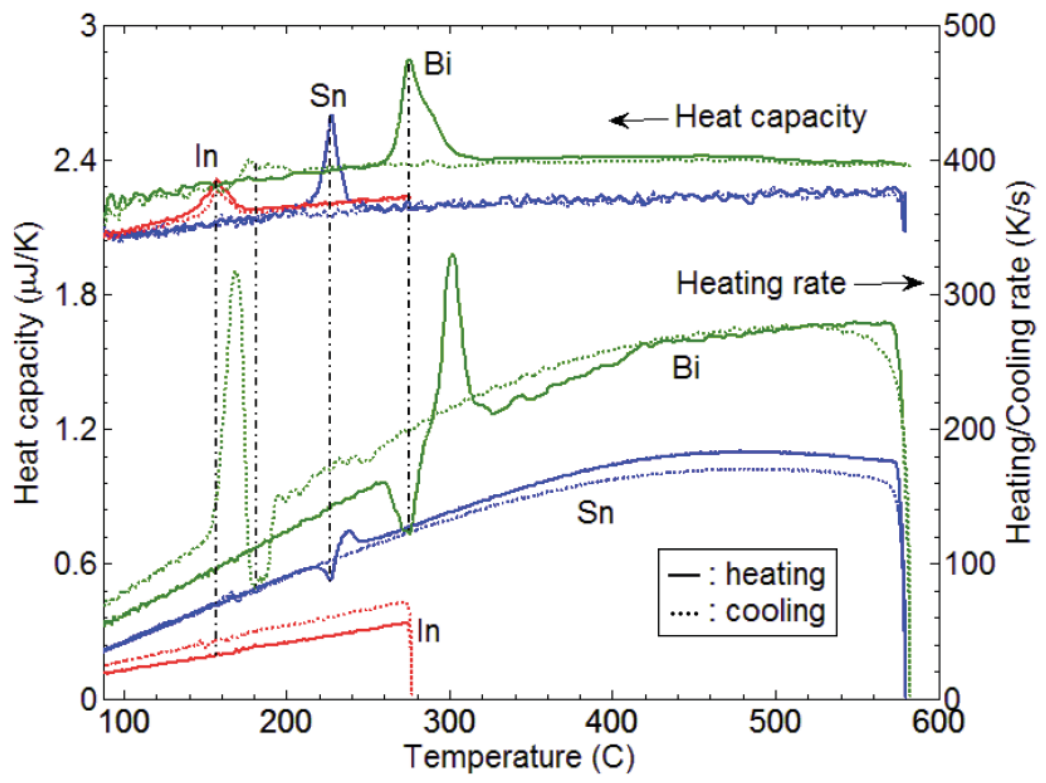

Figure 2.12. AC calorimetric signals on heating and cooling for sensors with $\mathrm{Sn}$, In, and Bi samples. The current $\left(I_{0}, i\right)$ for the Sn sample was increased linearly from $(30 \mathrm{~mA}, 26 \mathrm{~mA})$ to $(89 \mathrm{~mA}, 14 \mathrm{~mA})$, and then decreased to $(30 \mathrm{~mA}, 26 \mathrm{~mA})$. The current for In was increased from (31mA, 21mA) to $(86 \mathrm{~mA}, 59 \mathrm{~mA})$, and then decreased to $(13 \mathrm{~mA}, 9 \mathrm{~mA})$. The current for Bi was increased from $(20 \mathrm{~mA}, 20 \mathrm{~mA})$ to $(72 \mathrm{~mA} / 72 \mathrm{~mA})$, and then decreased to $(12 \mathrm{~mA} / 12 \mathrm{~mA})$. The heating rates refer to cycle-averaged heating rate, $\dot{\boldsymbol{T}}_{\mathbf{0}}$.

\section{Further discussion}

Nanocalorimetry combined with an AC technique makes it possible to obtain calorimetry data for very small quantities of materials over a broad range of heating and cooling rates, currently spanning the range from isothermal to $2 \times 10^{3} \mathrm{~K} / \mathrm{s}$. The limitation on the maximum scan rate is related to the data acquisition system, rather than the intrinsic properties of the nanocalorimetry device, and is set by the maximum AC frequency that yields an $\mathrm{AC}$ response that is both robustly measured within the sample rate of the $A D C$ and is sufficiently large to satisfy inequality (2.31). We can relax this 
latter constraint through use of a band pass filter as illustrated in the diagram in Figure 2.6. We have implemented an additional A/D channel in the data acquisition system. The sensor voltage signal is copied to this channel and sent through a band pass filter (SR640, Stanford Research Systems) that passes the harmonic signals and filters the relatively large DC and fundamental AC components. The resulting smaller amplitude signal is then amplified, resulting in a much smaller value of $\xi$. This option will be used in future measurements.

The ability to perform measurements over such a wide range of heating rates, essentially bridging the gap between traditional calorimetry and adiabatic nanocalorimetry, makes $\mathrm{AC}$ nanocalorimetry the ideal technique to study the behavior of materials far from equilibrium. We anticipate that the technique will be useful in the investigation of metallic glasses [29] and in elucidating the role of ultrafine microstructures in phase transformations, to name just a few examples. The broad range of heating rates also makes it possible to study the kinetics of phase transformations and solid-state reactions. Given that the technique is sensitive enough to probe thin-film samples and that it is straightforward to fabricate arrays of sensors, the technique also lends itself for high-throughput analysis of combinatorial libraries and composition spreads. Compared to combinatorial nanocalorimetry [7], the slower scan rates in AC nanocalorimetry make it possible to integrate technique with other characterization 
methods such as X-ray diffraction in a synchrotron, facilitating interpretation of the calorimetry traces.

\subsection{Summary}

We have presented an AC nanocalorimetry technique that enables calorimetric measurements on very small quantities of materials over a wide range of heating and cooling rates. Because of the small sample volumes involved, DC nanocalorimetry is quite sensitive to heat loss to the environment and very fast scan rates are required to approach adiabatic conditions. AC calorimetry, by contrast, is much less sensitive to heat loss and can also be used at slow scan rates, as long as the AC frequency is high enough. The AC technique relies on use of the PnSC device, which is an array of micromachined calorimeter sensors. This device is ideally suited for AC measurements because the small thermal impedance between the sample and the heater/thermometer allows the use of frequencies as high as $100 \mathrm{kHz}$. We have presented an analysis of AC calorimetry specified to the PnSC device and have derived conditions under which valid AC measurements can be obtained. The capability of the technique to make measurements using the PnSC device over a wide range of frequencies and heating/cooling rates is demonstrated by applying it to a $100 \mathrm{~nm}$ thin-film sample of Sn. 


\section{Chapter 3}

\section{Scanning AC nanocalorimetry combined with in-situ x-ray diffraction ${ }^{*}$}

\subsection{Introduction}

Nano-calorimetry is a recently developed materials characterization technique that has advanced rapidly in recent years $[2,3]$. It enables ultrasensitive calorimetry measurements on very small samples of materials through use of micromachined sensors with vanishingly small thermal mass. Due to the small thermal mass of the sensors, very fast heating rates can be achieved that minimize heat loss to the environment. For instance, the nanocalorimetry sensor originally developed by Allen and coworkers can attain heating rates as large as $10^{5} \mathrm{~K} / \mathrm{s}$, and has been used to perform very precise heat capacity measurements on nanoparticles $[4,52]$ and ultrathin polymer films $[15,41,53]$. Schick et al. have developed a different sensor that can operate under non-adiabatic conditions with controlled heating and cooling rates in the range of $10^{3}$ $\mathrm{K} / \mathrm{s}[16,54]$. A recent development in combinatorial nanocalorimetry by McCluskey and Vlassak expands the application of nanocalorimetry to material libraries with

\footnotetext{
${ }^{*}$ Reprinted with permission from K. Xiao, J. M. Gregoire, P. J. McCluskey, D. Dale and J. J. Vlassak, Journal of Applied Physics 113 (24), 243501 (2013). Copyright 2013, AIP Publishing LLC
} 
composition gradients, enabling high-throughput analysis of complex materials systems $[7,23]$. This combinatorial technique can be used to study the kinetics of phase transformations and the effects of heat treatments as a function of composition, and has been applied successfully to shape memory alloys and metallic glasses $[29,55,56]$.

An important challenge in the nanocalorimetric study of multi-component materials systems is to assign the features in the calorimetric trace to the various physical events that take place as a function of temperature - phase transitions in multi-component systems lead to complex calorimetric signals. One possible approach to resolve this challenge consists of simultaneously performing x-ray diffraction (XRD) and calorimetry measurements. XRD provides a wealth of structural information and allows identification of the phases present in a sample at a given temperature, while calorimetry yields thermodynamic and kinetic data that is highly temperature-resolved. In practice, combining both techniques is difficult due to the very different time scales involved in the techniques and the very small mass of the samples. For instance, to minimize heat loss, nanocalorimetry measurements are often performed at heating rates in excess of $10^{3} \mathrm{~K} / \mathrm{s}$ - a typical scan may last only a fraction of a second. XRD measurements, on the other hand, can require long exposure times and a typical $\theta-2 \theta$ scan on a thin-film sample may last hours. In this paper, we describe a new technique where we combine scanning AC nanocalorimetry with in-situ XRD measurements using synchrotron 
radiation.

Scanning AC nanocalorimetry [22] is a calorimetry technique in which an input power oscillation is superposed on a sample during a regular scanning calorimetry measurement. The amplitude and phase lag of the resulting temperature oscillation provide accurate information on both the heat capacity of the sample and its heat loss to the environment. Using this idea, Huth et al. ${ }^{16}$ and Guenther et al. ${ }^{17}$ developed AC chip-calorimeters that can operate at scanning rates below $10 \mathrm{~K} / \mathrm{min}[31,57]$. Xiao et al. [22] recently developed a different scanning AC nanocalorimetry technique that enabled measurements from nearly isothermal to rates as high as $2 \times 10^{3} \mathrm{~K} / \mathrm{s}$. This development, combined with the high intensity of a synchrotron $\mathrm{x}$-ray source, has made it possible to perform in-situ XRD measurements during a nanocalorimetry scan.

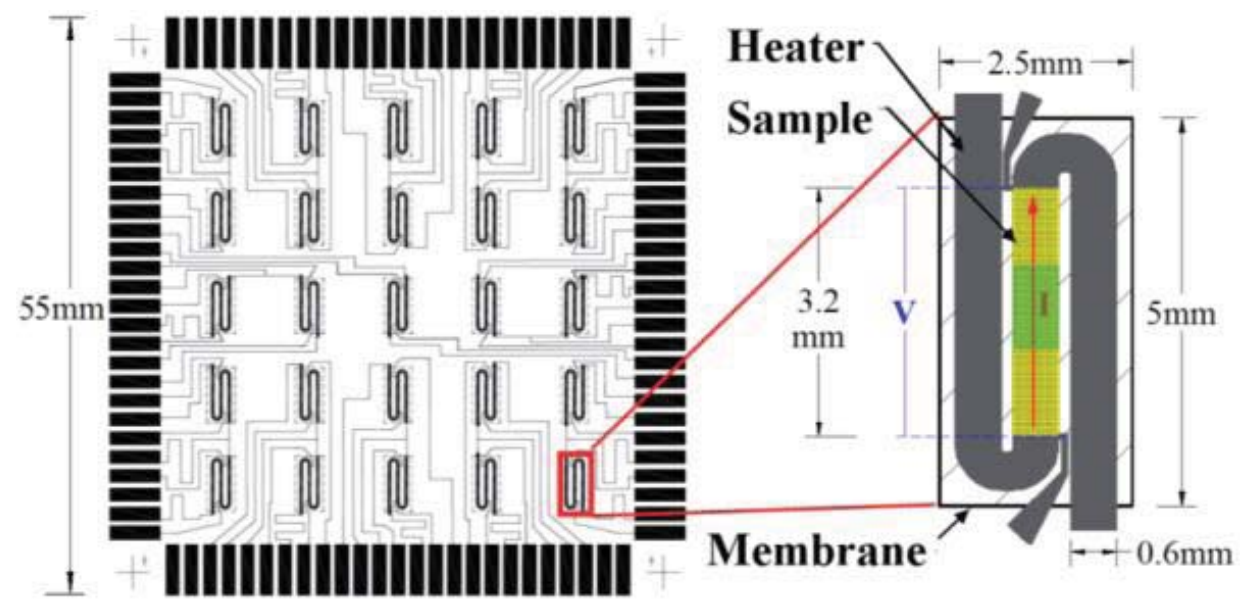

Figure 3.1. Schematic of the PnSC device and calorimetry sensor. The detailed view shows the metallization within the $2.5 \times 5 \mathrm{~mm}$ membrane. The sample deposition region shown in yellow is the same as the calorimeter measurement area. The subset of this area shown in green is the footprint of the $x$-ray beam. 
Here we provide a detailed description of the experimental technique and illustrate its capabilities by presenting preliminary measurements on a few simple materials systems. In Section 3.2 of this chapter, we describe the micromachined nanocalorimetry device that was used for the measurements and we provide a detailed account of the experimental set-up that was used to perform the XRD and calorimetry measurements at the synchrotron. In Section 3.3, we briefly review the theory that underlies scanning AC nanocalorimetry and provide the equations necessary for the analysis of the calorimetry data. This section also contains the details of the data reduction schemes. Results for experiments on thin-film samples of $\mathrm{Bi}, \mathrm{In}$ and $\mathrm{Sn}$ are discussed in Sections 3.4 and 3.5.

\subsection{Experimental setting}

\section{A. PnSC device}

The nanocalorimetry measurements in this study were performed using a parallel nano-scanning calorimeter (PnSC). The PnSC is a micromachined device described in detail elsewhere $[7,22,23]$. Briefly, it consists of a silicon substrate with a $5 \times 5$ array of independently controlled calorimeter sensors. Each sensor contains a tungsten four-point electrical probe that serves both as a heating element and a resistance thermometer. The tungsten probe is supported by a freestanding silicon nitride 
membrane and is completely encapsulated in silicon nitride. Figure 3.1 shows a schematic of the sensor that was used for AC calorimetry measurements in prior work [22]. In a typical calorimetry measurement, a thin-film sample is deposited in the shaded area between the two sensing leads and an electric current is supplied through the tungsten heating element. The measured current and voltage are used to determine the power supplied to the sample, while the temperature of the sample is determined from the resistance of the heating element, which is calibrated to temperature.

\section{B. Measurement set-up}

The XRD measurements were performed at the Cornell High Energy Synchrotron Source (CHESS) using a transmission-geometry diffraction experiment (see Figure 3.2), described elsewhere $[22,58]$. On the CHESS A2 beamline, a lightly sanded Si-111 double-crystal monochromator provided a $30 \mathrm{keV}$ x-ray beam with a flux of $4.5 \times 10^{10}$ photons per second in the $0.6 \times 1 \mathrm{~mm}$ beam cross-section. A large-area pixel array detector (GE 41RT) optimized for efficient detection of high-energy $x$ rays was used in transmission geometry to acquire diffraction data. The detector can image as fast as 30 frames per second.

To control the ambient atmosphere during the calorimetry measurement, the measurements were performed inside a small custom-built vacuum chamber with 
internal diameter of $30 \mathrm{~cm}$ to accommodate the probe card and PnSC device. To align the $x$-ray beam with each of the calorimeter samples in the $5 \times 5$ array, the entire chamber was mounted on a two-dimensional translation stage. The chamber contained an upstream x-ray window slightly larger than the PnSC device size and a considerably larger downstream window to avoid obstructing the diffraction cone from each calorimeter. Each window was custom made by sealing $0.1 \mathrm{~mm}$-thick polyimide film to a flanged aluminum frame using epoxy (Varian TorrSeal). The chamber was evacuated with a compact molecular drag turbo pump (Adixen MDP-5011) backed by a rotary vane pump (Edwards RV3). The chamber pressure was measured using thermocouple and ionization vacuum gauges (Kurt J Lesker 300 and 354). The chamber was evacuated to a base pressure of $10^{-6}$ Torr and measurements were performed either at this pressure or under a fixed pressure of $500-750$ mTorr of helium. 


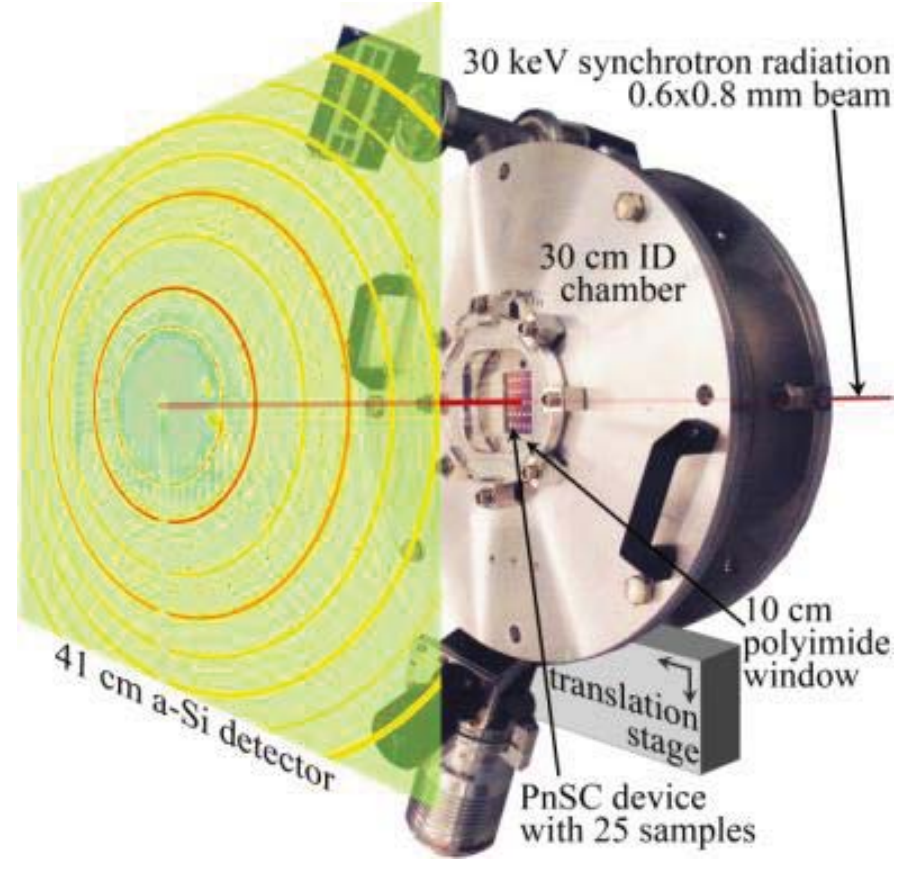

Figure 3.2. Experimental set-up used for the in-situ diffraction and nanocalorimetry measurements. The sample is located inside the chamber; the XRD measurements are performed in transmission geometry during the nanocalorimetry scan.

\section{Data acquisition system}

Nanocalorimetry measurements were performed using a data acquisition system controlled with Labview, along with a programmable current source. The systems consisted of several sub-systems as illustrated in Figure 3.3: (1) A current source for powering the PnSC sensors and for monitoring the current output; (2) a DAQ controller for acquiring the voltage output signals from the PnSC sensors; (3) an amplifier board for conditioning of the sensor's voltage response and (4) a band pass filter (Stanford Research Systems, SR640). 

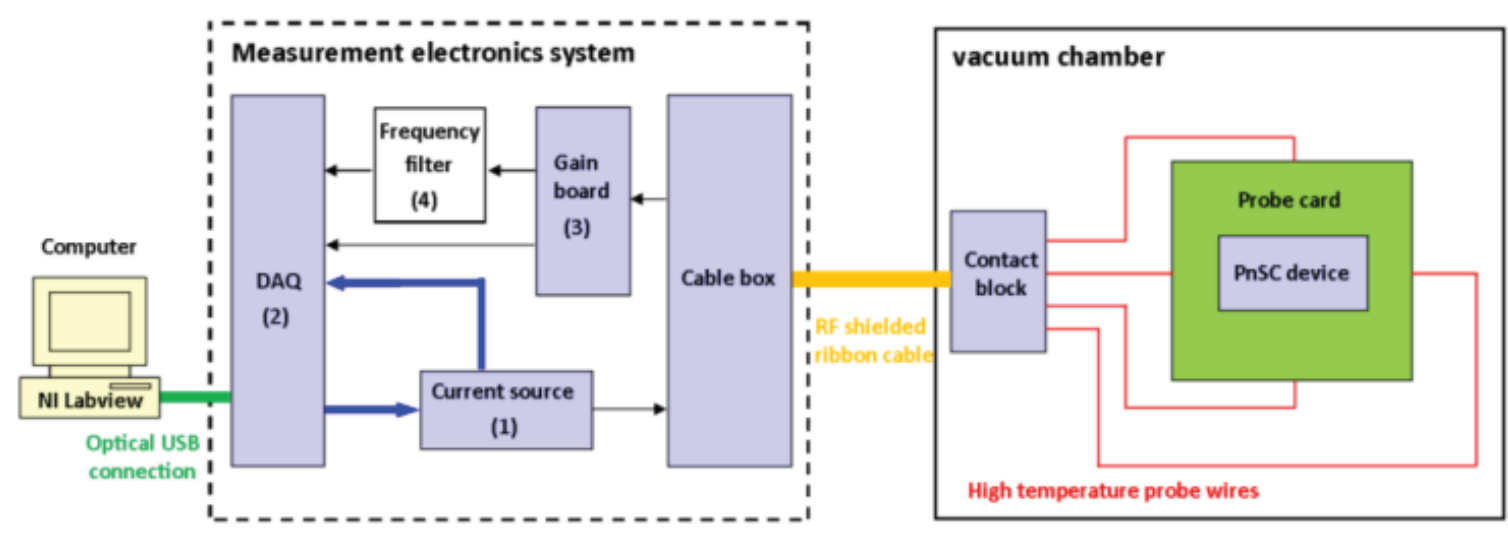

Figure 3.3..Schematic of the data acquisition system.

The data acquisition electronics setup was similar to that described in References [7]

and [22], with modifications to create a compact, portable system. Computer control and measurements were performed through a digital-to-analog (DA) and analog-to-digital (AD) module (National Instruments, NI cDAQ-9174 integrated with NI 9263 and NI 9205). The modified Howland current source consisted of three high-precision, low noise operational amplifiers (OPA2227, Texas Instruments, Austin, TX) and a high-output current differential driver (AD815, Analog Devices, Norwood, MA). The current source was driven by a $100 \mathrm{kHz}$ DA voltage output, providing capability for AC experiments up to $25 \mathrm{kHz}$. The applied current was determined by measuring the voltage across a precision resistor in series with the calorimeter device. The voltage across the calorimeter was measured directly, and this signal was also input into the band pass filter. The output of the band pass filter, the calorimeter voltage, and the precision resistor voltage were all sampled with the $A D$ module, using the 
pseudo-differential sampling mode for noise rejection. Noise rejection was also engineered into the electronics by isolating the DAQ module and current source from earth ground, which was accomplished with low-noise and ground-isolated DC power supplies (Agilent E3630A and E3620A) as well as galvanically isolating the DAQ communications over an optically isolated Universal Serial Bus (USB) interface to the computer. One voltage probe of the 4-point calorimeter cell was earth-grounded, and ground shields for all other cables were connected at this single ground terminal. Individual sensors on the PnSC device were selected by manually switching wire connections between the probe card and the data acquisition system. The measurement system did not have any feedback loop to control the sensor temperature; experiments were performed by applying pre-defined current profiles to the sensors. The integrated measurement system was controlled via a LabView program on the user computer. For a given experiment, both the calorimetry and XRD acquisition systems were triggered with a user-activated TTL signal and ran according to a pre-defined timing schedule.

\section{Sensor calibration and sample preparation}

After microfabrication of the PnSC device, each sensor was calibrated by measuring the resistance of the heating element and its linear temperature coefficient. The PnSC device was placed in a vacuum furnace and the furnace temperature was stepped from 
room temperature to $443 \mathrm{~K}$ in approximately $15 \mathrm{~K}$ intervals. The vacuum furnace was filled with helium gas to provide temperature uniformity within the chamber. At each temperature step, a DC current of $1 \mathrm{~mA}$, which yielded negligible Joule heating, was passed through each heating element on the PnSC device and the voltage across the heating element was recorded. The resistance of the heating elements was found to increase linearly with temperature over the entire temperature range of interest, in accordance with the previously detailed calibration of the resistance thermometers [7, 23]. Typical experimental values of the resistance, $R_{R T}$, and the thermal coefficient of resistance, $\lambda_{R T}$, at room temperature were $5.37 \pm 0.02 \Omega$ and $1.65 \mathrm{e}-3 \pm 2 \mathrm{e}-5 \mathrm{~K}^{-1}$, respectively.

Elemental Sn, Bi and In samples were deposited onto the sensors by magnetron sputtering in a centered, on-axis geometry. The depositions were performed using a shadow mask to limit deposition to the center segment of each serpentine sensor. The deposition parameters are summarized in Table 3.1. The total deposited mass was calculated from the mass accumulation rate, which was measured using a quartz crystal thickness monitor, and from the area of the openings in the shadow mask. The thickness values in Table 3.1 were calculated assuming standard densities of the elemental solids. After deposition, the shadow mask was removed and the samples were capped with a $32 \mathrm{~nm}$ aluminum nitride coating to protect the samples from the atmosphere during the 
calorimetry measurements. The coating was sputter deposited from an elemental Al target using a $100 \mathrm{kHz}$ pulsed DC power of $89 \mathrm{~W}$ in a $1.25 \mathrm{~Pa}$ atmosphere of $20 \% \mathrm{~N}_{2}$ in $\mathrm{Ar}$. During this deposition the PnSC device was rotated to attain conformal coating.

Table 3.1. Sample deposition parameters

\begin{tabular}{|c|c|c|c|c|c|}
\hline material & Ar pressure $(\mathrm{Pa})$ & Power $(\mathrm{W})$ & Rate $(\mathrm{nm} / \mathrm{s})$ & Thickness $(\mathrm{nm})$ & Mass $(\mu \mathrm{g})$ \\
\hline $\mathrm{In}$ & 1.3 & 11 & 0.25 & 190 & $2.6 \pm 0.4$ \\
\hline $\mathrm{Sn}$ & 2.4 & 6 & 0.14 & 100 & $1.4 \pm 0.2$ \\
\hline $\mathrm{Bi}$ & 0.67 & 15 & 1.1 & 300 & $5.6 \pm 0.5$ \\
\hline
\end{tabular}

\subsection{Theory and data reduction}

\section{A. Scanning AC calorimetry}

Scanning AC calorimetry measurements were performed by supplying a current,

$$
I=I_{0}+i \cos \left(\omega t-\varphi_{0}\right),
$$

to the heating element of a nanocalorimetry sensor and by measuring the corresponding $2 \omega$ voltage component across the voltage leads of the sensor (see Figure 3.1). The current induces a temperature rise along with temperature oscillations of frequencies $\omega$ and $2 \omega$. These oscillations cause the resistance of the heating element to oscillate, which in turn causes higher harmonics in the voltage response. The $2 \omega$ voltage response of the sensor is given by [22]

$$
\begin{aligned}
& V_{2 \omega}=X_{2 \omega} \cos (2 \omega t)+Y_{2 \omega} \sin (2 \omega t) \\
& X_{2 \omega}=\frac{i^{2} I_{0} R_{0}^{2} \lambda}{C \omega}\left[\sin \varphi_{1} \cos \left(2 \varphi_{2}+\varphi_{1}\right)+\frac{1}{4} \sin \varphi_{2} \cos \left(2 \varphi_{0}+\varphi_{2}\right)\right]
\end{aligned}
$$




$$
Y_{2 \omega}=\frac{i^{2} I_{0} R_{0}^{2} \lambda}{C \omega}\left[\sin \varphi_{1} \sin \left(2 \varphi_{2}+\varphi_{1}\right)+\frac{1}{4} \sin \varphi_{2} \sin \left(2 \varphi_{0}+\varphi_{2}\right)\right]
$$

where $C$ is the total heat capacity of the heating element and sample. $R_{o}$ and $\lambda$ are the temperature-dependent resistance and thermal coefficient of resistance of the heating element, respectively. The phase angles $\varphi_{1}$ and $\varphi_{2}$ are related by

$$
2 \tan \varphi_{1}=\tan \varphi_{2} .
$$

Detailed derivations of Eqs. (3.2) and (3.3) can be found in our previous work [22]. When the heat loss during the calorimetry measurement is large, the phase angles approach a value of $\pi / 2$, and the heat capacity $C$ is readily determined from Eq. (3.2). In the Appendix $B$, we show that $C$ can also be determined using the following more general approach. The phase angle $\varphi_{1}$ is given by the following equation,

$$
10 \tan ^{3} \varphi_{1}+9 n \tan ^{2} \varphi_{1}+4 \tan \varphi_{1}+3 n=0,
$$

where

$$
n=\frac{X_{2 \omega} \sin 2 \varphi_{0}-Y_{2 \omega} \cos 2 \varphi_{0}}{X_{2 \omega} \cos 2 \varphi_{0}+Y_{2 \omega} \sin 2 \varphi_{0}}
$$

It can be shown that Eq. (3.4) has only one real root, so that $\tan \varphi_{1}$ is uniquely determined by Eq. (3.4). The total heat capacity $C$ is then given by

$$
C=\frac{i^{2} I_{0} R_{0} k}{\omega \mid V_{2 \omega}} \mid \sqrt{\frac{25 \tan ^{2} \varphi_{1}+9}{16 \tan ^{2} \varphi_{1}+4} \times \frac{\tan ^{2} \varphi_{1}}{1+\tan ^{2} \varphi_{1}}} .
$$

Reference [22] provides a detailed discussion on how to select the experimental parameters $I_{0}, i$, and $\omega$ to perform valid AC calorimetry measurements. 


\section{B. Data reduction for AC calorimetry measurements}

To analyze the results, the measured currents and voltages for each experiment were divided into segments consisting of an integer number of AC oscillation periods. The $V_{2 \omega}$ voltage was evaluated by applying a discrete Fourier transform (DFT) to every segment. All discrete Fourier transforms were performed using a second order Goertzel algorithm, which is a very efficient method of computing DFT values at specific frequencies [42]. The temperature of the sensor was calculated from the resistance of the heating element and its thermal coefficient of resistance. The total heat capacity $C$ of a sensor with sample was calculated using Eqs. (3.4), (3.5), and (3.6). Finally, a window average method was implemented to smooth the heat capacity results. All calorimetry measurements presented in this paper include the heat capacity of the addendum, which was estimated at $1.8 \mu \mathrm{J} / \mathrm{K}$ from measurements performed on an empty sensor. The heat loss from sample to the environment increases with temperature, and was estimated to be $40 \mathrm{~mW}$ at $800 \mathrm{~K}$ [22].

\section{XRD measurement}

For each experiment, the $\mathrm{x}$-ray beam was aligned to the center of the calorimeter sensor as illustrated in Figure 3.1. The sample mass for the $\mathrm{x}$-ray measurement was on the order of $1 \mu \mathrm{g}$, approximately one third of the calorimetry sample mass (see Table 3.1). For these experiments, $x$-ray detector frame rates between 0.1 and $10 \mathrm{~Hz}$ were 
used for crystallographic characterization of the sample as functions of time and temperature; the acquisition times are provided in the relevant figure captions. The combination of small sample mass and short integration time pushed the detectability limit of the diffraction measurement, requiring careful data processing for quantitative analysis. The aggregate background scattering from the air, the polyimide windows, etc. was characterized by acquiring diffraction patterns on a PnSC substrate with no device. After subtracting this background and performing azimuthal integration to produce $\mathrm{x}$-ray powder patterns, the sequence of patterns for a given experiment were analyzed in aggregate by summing the patterns. The Bragg peaks identified in the sum pattern were used to define segments of the powder pattern, which were analyzed in each pattern in the sequence. For each experiment, the diffraction patterns acquired near the highest sample temperature were analyzed to confirm the absence of sample Bragg peaks, and then the average of these patterns was used as a background pattern for the sequence of images. This background subtraction method effectively removed the scattering from the tungsten heating element. Before and after each experiment, long-exposure diffraction images were also acquired to provide characterization of the room-temperature sample. 


\subsection{Results}

Typical AC calorimetry results obtained for the Bi thin-film samples are shown in Figure 3.4. Figure 3.5 shows the corresponding Bi XRD patterns obtained at different temperatures during the calorimetry scans. The heat capacity in Figure 3.4 reflects the true heat capacity of the Bi sample, i.e., the energy added or extracted to reversibly heat or cool the sample without any heat loss to the environment [22]. Heating and cooling scans obtained in three different measurements are shown. The data from the three different measurements lie on top of each other, demonstrating excellent reproducibility. Both the heating scans (solid curves) and the cooling scans (dashed curves) show distinct peaks caused by melting or solidification of the sample. Unlike DC calorimetry measurements, these peaks are not directly related to the latent heat of melting $[17,45]$. On heating, the $\mathrm{AC}$ component of the power supplied to the sample during the melting process can create oscillations in the relative fraction of the two phases (i.e., movement of the phase boundary), which leads to reduced temperature oscillations and results in a distinct peak in the $A C$ calculation of the heat capacity $[17,22]$. These peaks can be used to determine the melting temperature of the sample and are related to the reversibility of the reaction at the time scale of the temperature oscillation, but the area under the peak does not correspond to the latent heat of melting. The widths of the peaks are determined mainly by the temperature uniformity of the sensor and the amplitude of 
temperature oscillation [22]. Here, the melting temperature is taken at the maximum point of the heat capacity signal $[7,22]$. The value for $\mathrm{Bi}$ is found to be $543.3 \mathrm{~K}$, in good agreement with the literature value of $545.1 \mathrm{~K}$ [38]. The solidification peaks on cooling, however, occur at $448.7 \mathrm{~K}$, which is significantly lower than the equilibrium melting temperature of $\mathrm{Bi}$. The corresponding peaks in the calorimetry trace are not nearly as distinct as those on heating since the temperature oscillations are not reduced during solidification as much as they are during melting. Because of the undercooling, the solidification process is a non-equilibrium process with a rate of solidification that is set by the degree of undercooling and that has a much smaller effect on the temperature oscillations compared to melting. At temperatures between the melting and solidification points, there is a small but distinct offset between the heat capacity curves obtained on heating and cooling, indicating that the super-cooled liquid Bi has a slightly higher heat capacity than solid $\mathrm{Bi}$ at the same temperature. This finding is in agreement with measurements on bulk Bi samples [59]. 


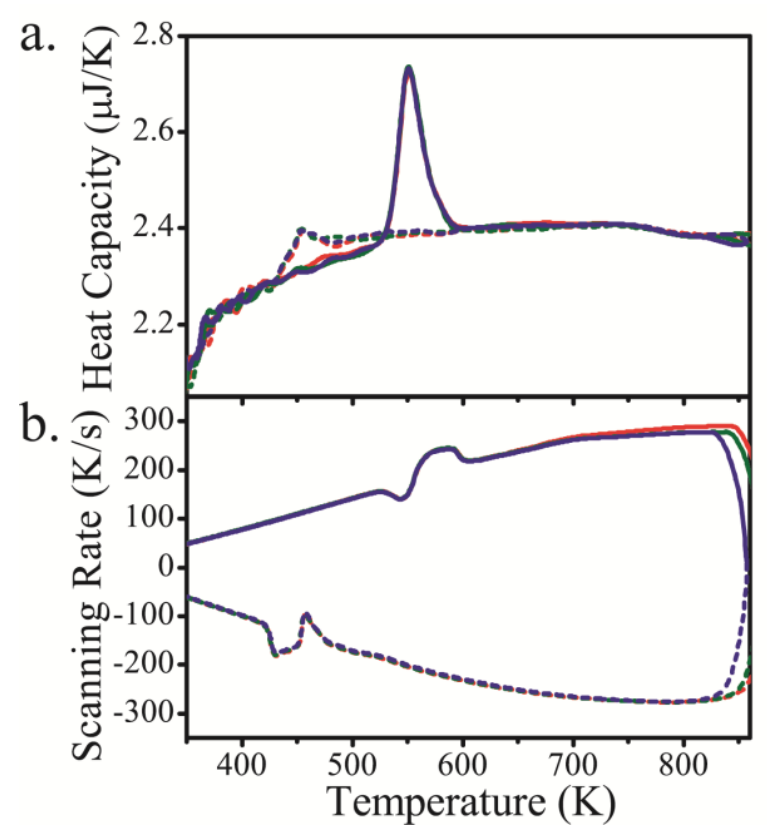

Figure 3.4. (a) Heat capacity and (b) corresponding scanning rates as a function of temperature for Bi for three calorimetry scans (solid lines: heating, dashed lines: cooling). The current $\left(I_{0}, i\right)$ used for the measurements was increased linearly from $(20 \mathrm{~mA}, 20 \mathrm{~mA})$ to $(72 \mathrm{~mA}, 72 \mathrm{~mA})$, and then decreased to $(12 \mathrm{~mA}, 12 \mathrm{~mA})$. Measurements were performed using a frequency of $416.6 \mathrm{~Hz}$. The amplitude of temperature oscillation is $6.1 \mathrm{~K}$ at $500 \mathrm{~K}$.

Figure 3.5 shows several Bi XRD images taken at different temperatures during the heating and cooling scans of the AC calorimetry measurement. The diffraction geometry is similar to that used in transmission electron microscopy and the ensuing diffraction patterns indeed resemble those obtained in a transmission electron microscope. Due to the random crystal texture, grains diffract into Debye rings, which typically exhibit a uniform intensity distribution due to the multitude of grains in the diffracting sample volume. While the Debye rings for the metal samples contain some uniform component, samples solidified from a melt contain larger grains that produce bright spots in the Debye ring when they satisfy the Bragg condition. For the Bi sample, the images in 
Figure 3.5 show Debye rings with approximately 100 bright spots, and the same approximate number of spots is observed in each $\{\mathrm{hkl}\}$ ring due to the random texture. Debye rings for the thinner In sample (not shown) contain only about 10 bright spots. Since the number of grains in the diffraction sample is not sufficiently large to create uniform powder diffraction rings, the use of a linear (one-dimensional) detector would fail to capture a powder pattern.

On heating, the diffraction patterns obtained at temperatures below the melting point are well matched to that of rhombohedral $\mathrm{Bi}$, while those obtained above the melting point do not contain detectable Bragg peaks. On cooling below the solidification temperature the Bi diffraction pattern reappears. The integrated diffraction intensity of the $\mathrm{Bi}\{110\}$ reflection is shown as a function of temperature in Figure 3.6, clearly showing melting and solidification reactions. We also analyzed the diffraction images to ascertain the evolution of the crystal orientation distribution during the calorimetry experiment. For each image, a window of scattering vector magnitude containing the $\mathrm{Bi}$ $\{110\}$ scattering was integrated along the scattering vector magnitude to yield the diffraction intensity as a function of detector azimuth, which is equivalent to the azimuthal orientation of the crystal grains on the sample. We quantify the evolution of this pattern as a function of temperature by calculating the correlation with the room-temperature diffraction pattern from before and after the calorimetry experiment 
$\left(C_{i}(T)\right.$ and $C_{f}(T)$, respectively). These correlations are shown in Figure 3.6 and contain three important features. First, the sharpest transitions in the correlations occur at the melting and solidification reactions as the orientation patterns of the liquid sample contain no diffraction intensity, yielding a vanishing correlation value. The most important feature of these correlations is that $C_{f}(T)$ is small before the melting reaction and $C_{i}(T)$ is small after the solidification reaction. This observation implies that there is no measurable correlation between the orientations of the grains before and after melting, as might be the case if grains had fixed nucleation sites that would result in preferred orientations. Finally, both $C_{i}(T)$ and $C_{f}(T)$ gradually decrease from their unity value with increasing temperature. Over the temperature range of these experiments, the relative thermal expansion of the samples is on the order of $10^{-3}$. The magnitudes of the scattering vectors of the Bragg peaks shift by a corresponding amount, but these shifts are not generally detected because they are much smaller than the observed peak widths and are comparable to the resolution of the diffraction measurement. Thus thermal shifts do not contribute to the decrease in correlation with increasing temperature. Closer analysis of the orientation distribution curves (not shown) reveals that the decrease in correlation is caused by variations in the peak heights compared to the room-temperature diffraction pattern. These variations in peak heights may be caused by grain growth in the sample, which would not be surprising given the low 
melting point of Bi. Abrupt disappearance of a peak or appearance of a new peak, indicative of recrystallization of the sample, is not generally observed. The analysis of the structural evolution of the sample highlights the depth of information that can be obtained by combining a thin film heater/thermometer and a high-energy diffraction experiment using an area detector.
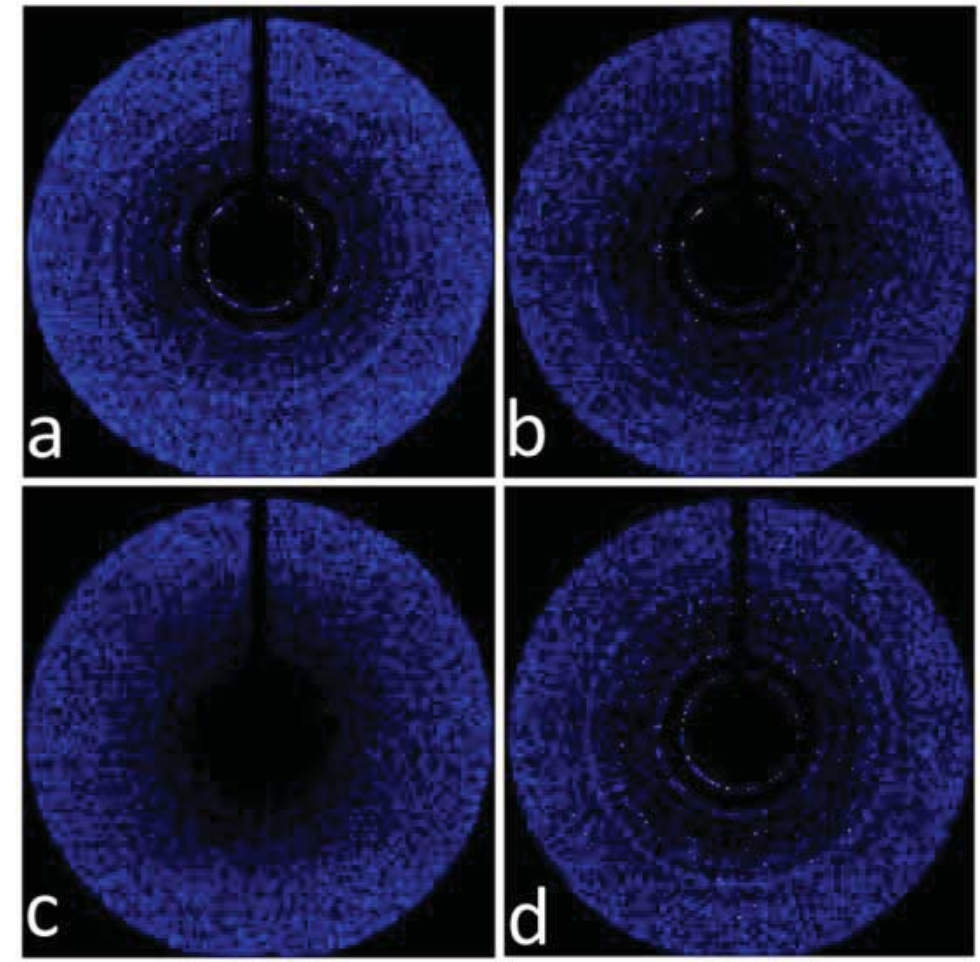

Figure 3.5. XRD images taken for the Bi sample at different temperatures during the nanocalorimetry scans. (a) $373 \mathrm{~K}$; (b) $473 \mathrm{~K}$; (c) $673 \mathrm{~K}$ (above melting point) (d) $373 \mathrm{~K}$ (after solidification). The XRD image acquisition time was $0.1 \mathrm{~s}$. 


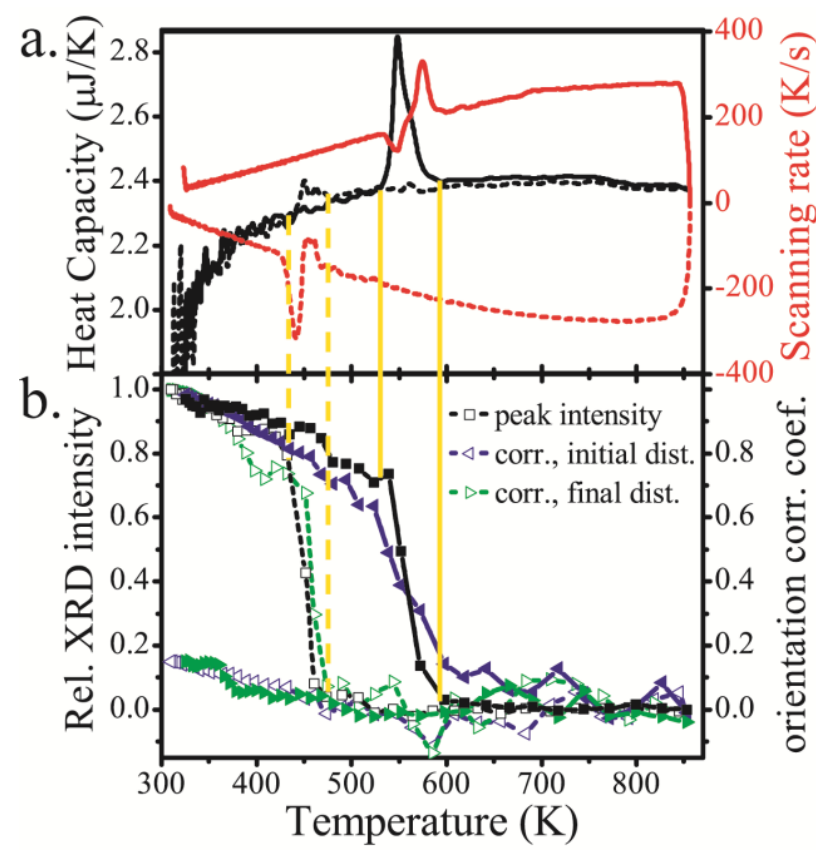

Figure 3.6. (a) Heat capacity and heating rate for the Bi sample as a function of temperature; the experimental parameters are the same as in Figure 3.4; (b) Correlation of diffraction patterns and peak intensity as a function of temperature. Solid lines are for heating, dashed lines are for cooling. The vertical yellow lines mark alignment of the reaction windows in the PnSC and XRD signals. The image acquisition time was 0.1s.

The AC calorimetry and XRD results obtained for the In sample are presented in Figure 3.7. Results for two identical scans are shown. The calorimetry data from both measurements are nearly identical, demonstrating excellent reproducibility. Both the heating scans (solid curves) and the cooling scans (dashed curves) show distinct peaks caused by In melting and solidification, respectively. The melting temperature is found to be $432.7 \mathrm{~K}$, in good agreement with the literature value of $429.7 \mathrm{~K}$ [38]. Unlike Bi, however, melting and solidification of In occur at the same temperatures - no undercooling is observed in Figure 3.7 and the solidification proceeds in a reversible manner. As a result, the temperature oscillations are reduced both on rising and falling, 
and the calorimetry traces for solidification and melting are quite similar. Figure 3.7 also shows the intensity of the In $\{101\}$ peak as a function of temperature. The change in diffraction intensity with melting and solidification is readily discerned, but compared to $\mathrm{Bi}$ the data are less reproducible and much noisier because of the smaller sample mass and atomic form factor of In. In the second experiment, for instance, the peak intensity briefly jumps to a very large value as a single large grain of In momentarily satisfies the Bragg condition. This phenomenon of course also occurs for $\mathrm{Bi}$, but the smaller sample mass and possibly larger grains of In result in much larger statistical fluctuations.
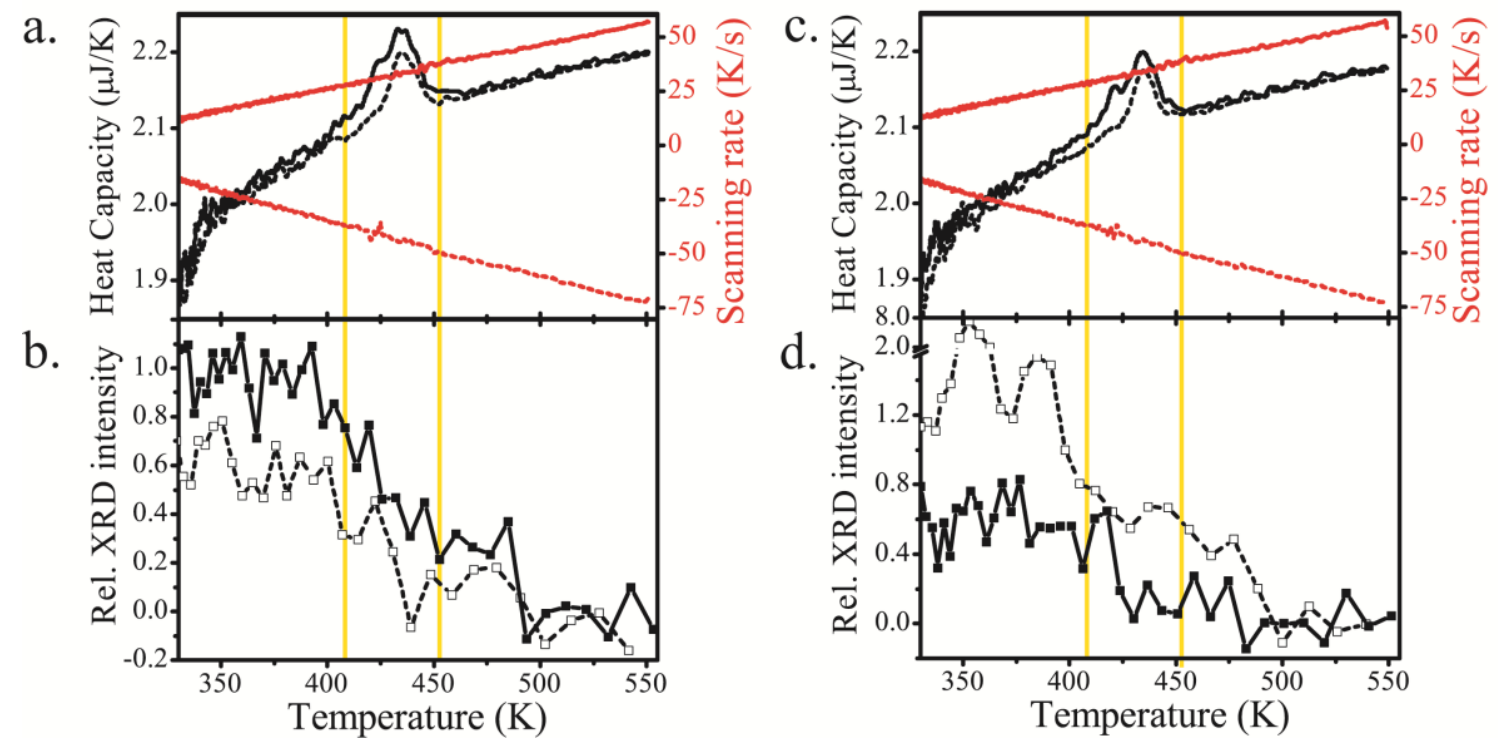

Figure 3.7. Heat capacity, scanning rates and XRD intensity for the In sample as a function of temperature for two different scans (solid lines: heating, dashed lines: cooling). (a), (b) are results for the 1st measurement and (c), (d) for 2nd measurement. The current $\left(I_{0}, i\right)$ used for the two measurements was increased linearly from $(27 \mathrm{~mA}, 18 \mathrm{~mA})$ to $(86 \mathrm{~mA}, 59 \mathrm{~mA})$, and then decreased to $(13 \mathrm{~mA}, 9 \mathrm{~mA})$. The AC frequency used was $416.6 \mathrm{~Hz}$ for both scans. The amplitude of temperature oscillation is $9.9 \mathrm{~K}$ at $500 \mathrm{~K}$. The XRD image acquisition time was 0.1s.

Calorimetry and in-situ XRD results obtained for the Sn sample are shown in Figure 
3.8. The figure contains data for two sets of measurements performed at two different scan rates. The heating scans (solid curves) in the calorimetric signal show a distinct melting peak at $502.4 \mathrm{~K}$ for the fast scan, and at $483.4 \mathrm{~K}$ for the slow scan. The former value agrees well with the literature value of $505 \mathrm{~K}$; we attribute the offset in the slow scan to the very large temperature oscillation used in this scan ( $27 \mathrm{~K})$, which was an inadvertent result of the smaller AC frequency used to perform the measurement. In contrast to the heating scans, the cooling scans (dashed curves) do not have any obvious features associated with solidification. This absence of a distinct solidification peak for Sn was also observed in previous nanocalorimetry measurements [22].

The XRD results shown in Figure 3.8 were obtained using an image acquisition time of $0.1 \mathrm{~s}$ for the fast scan, and $2 \mathrm{~s}$ for the slow scan - the much-improved signal-to-noise ratio for the slower scan is evident. Unlike the calorimetry traces, the XRD signals show clear transitions on both heating and cooling. The solidification process starts at the melting point and completes over a temperature range of approximately $150 \mathrm{~K}$. This observation suggests a gradual solidification process in which isolated Sn droplets on the sensor solidify at different degrees of undercooling. This process is consistent with previous nanocalorimetry measurements on individual drops of $\mathrm{Sn}$ that showed size-dependent undercooling $[60,61]$ in excess of $100 \mathrm{~K}$. The gradual character of the solidification process explains the absence of any distinguishing features in the cooling 
segments of the calorimeter traces because the temperature oscillations are averaged over the entire area of the sensor.
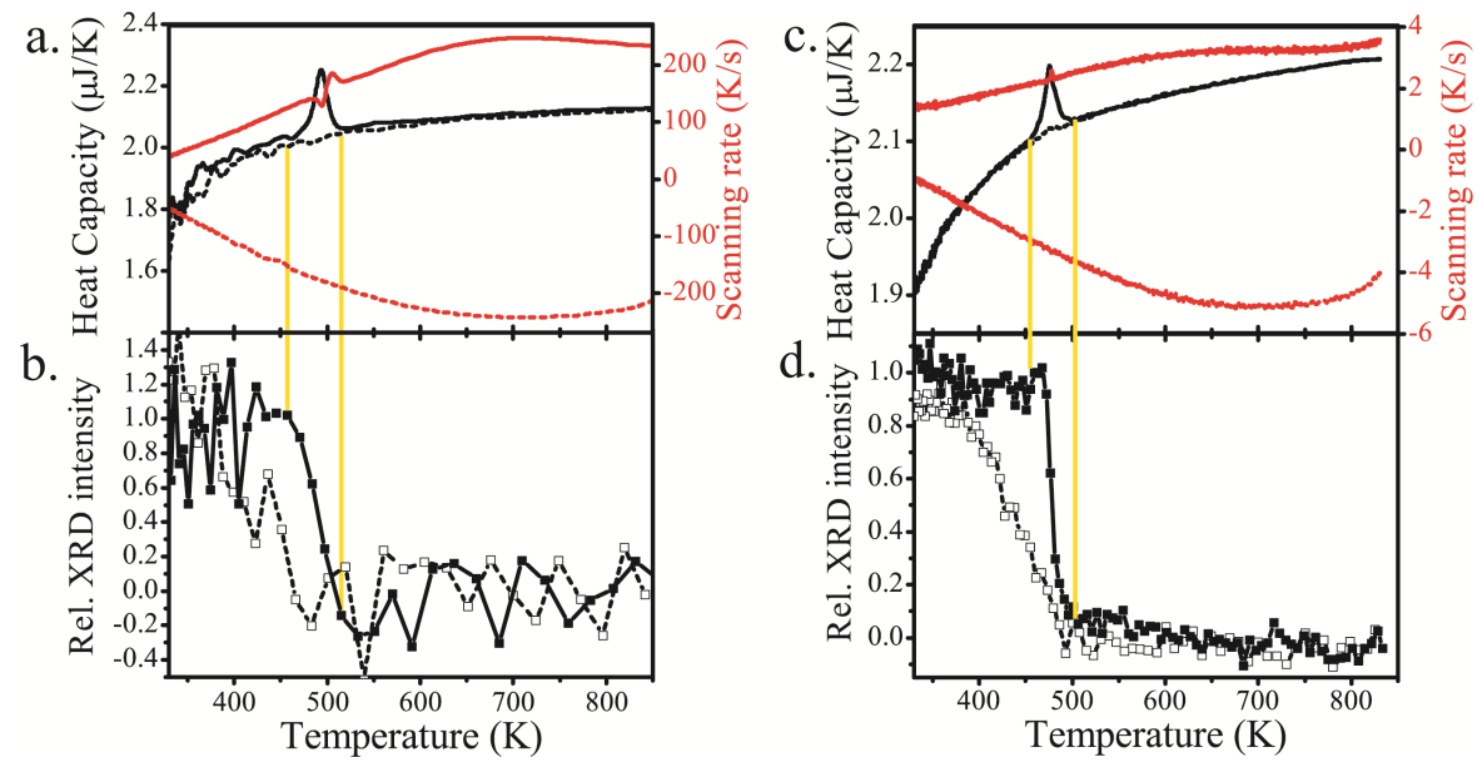

Figure 3.8. Heat capacity, scanning rates and XRD intensity as a function of temperature for fast and slow scans on Sn (solid lines: heating, dashed lines: cooling). (a), (b) are results for the fast measurement and (c), (d) for slow measurement. The current $\left(\mathrm{I}_{0}, \mathrm{i}\right)$ used for the fast scan was increased linearly from $(20 \mathrm{~mA}$, $20 \mathrm{~mA})$ to $(72 \mathrm{~mA}, 72 \mathrm{~mA})$, and then decreased to $(12 \mathrm{~mA}, 12 \mathrm{~mA})$. The current $\left(I_{0}, \mathrm{i}\right)$ used for the slow scan was increased linearly from $(8 \mathrm{~mA}, 8 \mathrm{~mA})$ to $(71 \mathrm{~mA}, 71 \mathrm{~mA})$, and then decreased to $(5 \mathrm{~mA}, 5 \mathrm{~mA})$. The $A C$

frequency used was $416.6 \mathrm{~Hz}$ for the fast scan and $99.2 \mathrm{~Hz}$ for the slow scan. The amplitude of temperature oscillation at $500 \mathrm{~K}$ is $5.9 \mathrm{~K}$ for fast scan and $27.7 \mathrm{~K}$ for slow scan. The XRD image acquisition time was $0.1 \mathrm{~s}$ for fast scan and $2 \mathrm{~s}$ for slow scan.

\subsection{Discussion}

The nanocalorimetry and XRD measurements on the $\mathrm{Bi}$, In and $\mathrm{Sn}$ samples suggest that they have rather different nucleation behavior. The Bi sample shows significant undercooling and solidifies over a narrow temperature range. The Sn sample, in contrast, starts to solidify at the melting temperature, but the process is stretched out over a 
broad range of temperatures. The In sample does not show any undercooling, even at cooling rates as fast as $100 \mathrm{~K} / \mathrm{s}$. The behavior of the In sample suggests that the energy barrier to nucleation of the solid phase is very small, i.e., nucleation occurs heterogeneously and there is strong catalytic activity of either the underlying $\mathrm{SiN}_{\mathrm{x}}$ layer or the $\mathrm{AlN}_{\mathrm{x}}$ capping layer. The behavior of the Bi sample is more typical of a transition that occurs via a classical nucleation and growth mechanism. The undercooling is necessary to overcome the nucleation barrier, but once the solidification process starts, it progresses quickly. The solidification of the Sn sample occurs via a similar nucleation and growth mechanism, but the gradual character of the solidification process suggests that in this case the film consists of individual islands with a range of sizes and with size-dependent solidification temperatures. Solidification of one island does not imply solidification of the entire film, as the islands are isolated from each other. The result is a solidification process with a temperature-dependence that is determined by the size distribution of the islands. This explanation is consistent with the microstructure of sputtered Sn films since Sn films are known to form discrete islands over a broad range of sputtering conditions [62] and the observation that melted Sn does not wet the $\mathrm{SiN}_{\mathrm{x}}$. Figure 3.9 shows an optical micrograph of a Sn sample subjected to many melting cycles. The island-structure of the sample is readily discerned. It should be noted that the size effect is a kinetic effect distinct from size-dependent melting point depression because it 
is observed only during solidification and not during melting.

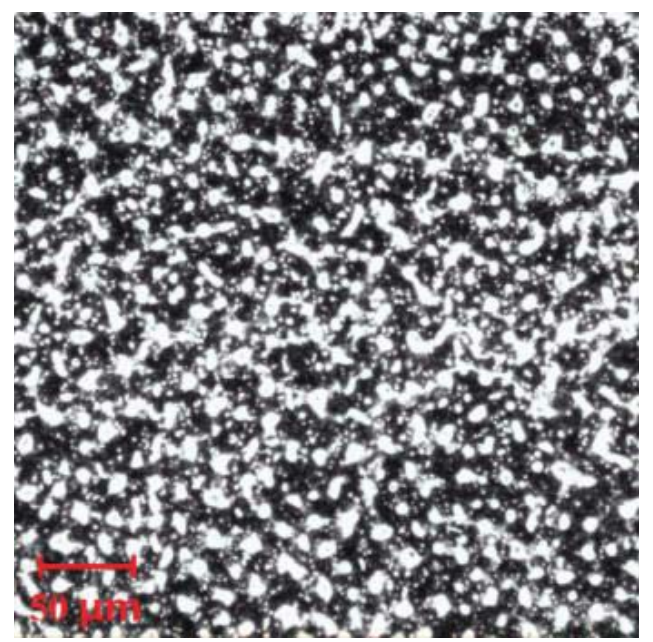

Figure 3.9. Optical image of Sn sample aftre many melting cycles.

Nucleation of a solid phase in a melt is a stochastic process. The stochastic nature can be used to obtain information on the underlying nucleation kinetics by measuring the statistical fluctuations in the undercooling. When a melt is cooled down below its melting point, the undercooling at which solidification occurs typically follows a Poisson distribution and the distribution of undercooling values, typically over a 10-20 K range $[60,63-65]$, can be used to derive the nucleation rate as a function of temperature [66, 67]. In the present case, the $\mathrm{Bi}$ and In solidification peaks are highly reproducible, indicating a more deterministic solidification process. This deterministic appearance occurs because solidification of a thin-film sample is not the result of a single nucleation event, but of a very large number of events. The very large interface-to-volume ratio of a thin film provides ample opportunity for heterogeneous nucleation as confirmed by the 
polycrystalline structure of the samples after solidification. By contrast, many studies of metal undercooling go to great lengths to minimize nucleation through use of small spherical samples suspended in vacuum or surrounded by a flux [60, 63-65]. Because so many nucleation events occur during solidification of a thin-film sample, the calorimetry trace can be regarded as a cumulative superposition of traces obtained for many individual nucleation events, and analysis of the shape of the solidification peak should provide information on nucleation statistics. There are, however, a number of other factors that influence the shape of this peak including the temperature uniformity across the sample area and the temperature oscillations of the AC measurement [22], making the analysis somewhat involved and beyond the scope of the present manuscript. A direct comparison of the melting and the solidification peak may afford insight in nucleation statistics, provided the temperature uniformity is the same on heating and cooling. In this context, a DC calorimetry measurement would be preferred over an AC measurement because current oscillations affect the temperature of the sample differently during melting and solidification under super-cooled conditions. It is possible, however, to draw qualitative conclusions based on the calorimetry data presented above. The nucleation barrier for the In sample is very small and no undercooling is observed. The solidification and melting peaks in this case are determined mainly by the temperature uniformity and the $\mathrm{AC}$ oscillations, and indeed they have very similar 
shapes. The Bi sample shows significant undercooling and analysis of the solidification peak would provide insight in the statistics of the nucleation process, at least if the effect of the AC oscillations could be eliminated. The calorimetry signal for the solidification of the Sn sample is not strong enough to allow for a meaningful analysis, but the XRD data combined with a detailed size distribution could provide information on the size-dependence of the solidification temperature.

In-situ XRD measurements prove very useful in identifying features in the calorimetry trace such as the melting and solidification peaks in this work. Once the calorimetry features have been identified, the associated phase transformations or solid-state reactions can be investigated as a function of heating/cooling rate, heat treatment, or even composition by employing the PnSC combinatorial capability. We also note that while an inert helium atmosphere was employed in this study, the use of reactive gasses could be used to study solid-gas reactions. The large surface-to-volume ratio of the thin-film format and the ability to study thin films over a range of thicknesses will provide very high sensitivity in measurements of surface reactions. The main limitations for in-situ XRD measurements are currently the small masses of the samples and the very short exposure times - the scattered intensity is relatively low and requires careful analysis. This situation can be improved through use of more intense x-ray beams and faster x-ray detectors. 


\subsection{Summary}

Scanning AC nanocalorimetry can be performed on thin-film samples over a wide range of scanning rates. This feature makes it possible to combine the calorimetry technique with in-situ X-ray diffraction measurements, allowing simultaneous structural and thermal characterization of the samples as a function of temperature. We have applied the technique to thin-film samples of $\mathrm{Bi}$, In and $\mathrm{Sn}$. The melting and solidification behavior of the samples is readily observed and our experiments show that the solidification process is distinctly different in each of the three samples.

Nanocalorimetry and in-situ XRD provide complementary information on the phase transformations that occur in a sample. While the application to melting and solidification of elemental metals is relatively straightforward, we anticipate that the technique will be most useful in the analysis of complex materials systems where identification of features in the calorimetry trace is often not a trivial exercise. Combining XRD and nanocalorimetry experiments provides unprecedented capability for studying the kinetics of phase transformations or solid-state reactions, and the evolution of intermediate phases. 


\section{Chapter 4}

\section{Nucleation behavior of melted Bi films at cooling rates from $10^{\wedge} 1$ to $10^{\wedge} 4 \mathrm{~K} / \mathrm{s}$ studied by combining scanning $A C$ and $D C$ nano-calorimetry techniques ${ }^{*}$}

\subsection{Introduction}

The undercooling of liquid metals and alloys below their thermodynamic freezing temperature has been a topic of interest for many years $[63,64,68-73]$. The degree of undercooling and the nucleation process that initiates the liquid-to-solid phase transformation greatly influence the ensuing microstructure and properties of a material. Due to the stochastic nature of the nucleation process, a single measurement of the undercooling response of an individual droplet does not provide sufficient information to determine the kinetic parameters. Instead, the kinetics of solidification is studied by measuring a large number of independent nucleation events in dispersions of similar individual droplets $[70,71,74-78]$, or by measuring nucleation in a single droplet multiple times using the same temperature trace $[60,63,64,79,80]$. While the single droplet method provides a direct observation of individual nucleation events, the

\footnotetext{
${ }^{*}$ Reprinted with permission from K. Xiao and J. J. Vlassak, Thermochimica Acta 603 (0), 29-38 (2015). Copyright 2015, Elsevier
} 
droplet dispersion method guarantees identical thermal histories for all droplets and is more efficient, especially when the numbers of droplets in the dispersion is large $\left(>10^{3}\right)$. The nucleation rate can then be determined from the distribution of undercooling values obtained for a fixed cooling rate $[63,64,66]$, or by measuring the undercooling as a function of cooling rate $[70,78]$.

Recent developments in nano-calorimetry have enabled very fast scanning measurements at cooling rates that are not accessible with traditional differential scanning calorimeters or differential thermal analyzers. For instance, Schick et al performed measurements on single droplets of Sn over a wide range of cooling rates (100 to $14,000 \mathrm{~K} / \mathrm{s})$ using a chip-calorimeter $[60,61]$. Because these measurements are performed on single droplets, they require many replicas at each cooling rate to ensure statistical significance. Here we combine chip-calorimetry with thin-film deposition techniques to fabricate the samples. When a very thin metal film is deposited on a substrate with high interfacial energy and then melted, the film breaks up into a large number of very small isolated islands to minimize the interfacial energy between metal and substrate. The film thus forms a dispersion of individual droplets that is ideal for nucleation studies. This approach has the advantage of being able to measure a large set of similar droplets in a single scan. We perform calorimetry measurements using a nanocalorimeter sensor derived from an original design by Allen and coworkers [3, 15], 
which has negligible thermal lag between sample and temperature sensor thus enabling accurate temperature measurement at very high scanning rates. This nanocalorimeter sensor combined with the appropriate AC-technique [22] is also capable of making accurate measurements at medium to slow scan rates where heat loss to the environment makes DC measurements not practical [81], even allowing in-situ XRD measurements during the scans [26]. By combining $A C$ and $D C$ techniques we have performed measurements on undercooled $\mathrm{Bi}$ samples at cooling rates from $10^{1}$ to $10^{4}$ $\mathrm{K} / \mathrm{s}$ and use classical nucleation theory to interpret the experimental results.

The main text of this chapter is organized into four sections. In Sec. 4.2, we introduce the nanocalorimetry chip that was used for the measurements and describe the measurement setup. In Sec. 4.3, we describe a data reduction technique that combines DC and AC calorimetry results to study reaction kinetics. We then apply this technique to classical nucleation theory in Sec. 4.4. Results from the nucleation experiments on thin-film samples of Bi are discussed in Secs. 4.5 and 4.6.

\subsection{Experimental detail}

The calorimetry measurements in this study were performed using a micromachined nano-calorimetry sensor that has been described in detail elsewhere [7, $22,23]$. Briefly, the sensor consists of a tungsten four-point electrical probe that serves 
both as a heating element and a resistance thermometer. The tungsten probe is completely encapsulated in silicon nitride and is supported by a freestanding silicon nitride membrane, which in turn is supported by a silicon frame. Figure 4.1 shows a schematic of the sensor. In a typical measurement, a thin-film sample is deposited in the shaded area between the two voltage sensing leads and an electric current is applied to the tungsten heating element, which, in turn, heats the sample. The measured values of current and voltage are used to determine the power supplied to the sample, while the temperature of the sample is determined from the resistance of the heating element, which is calibrated to temperature using a standard procedure described in detail in references $[22,26]$.

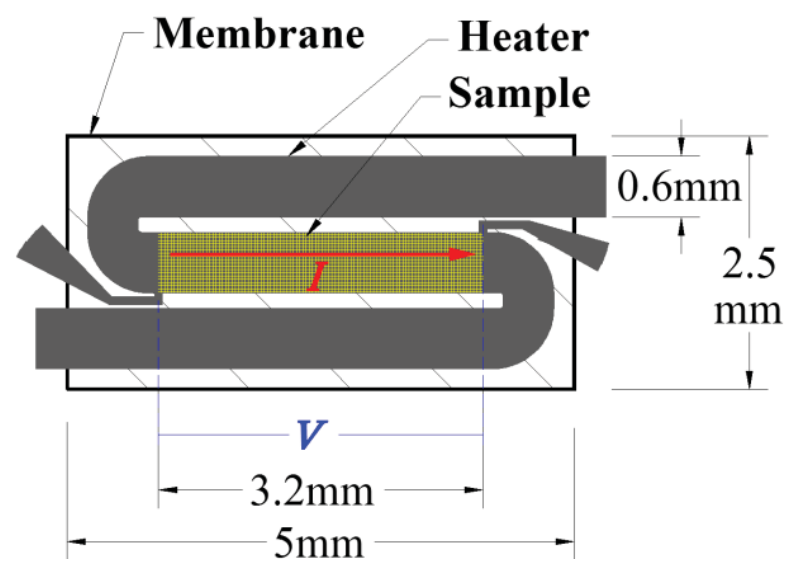

Figure 4.1. Schematic of the calorimetry sensor. The sample deposition region shown in yellow is the same as the calorimeter measurement area.

The nanocalorimetry sensor is installed in a probe card that is mounted inside a high vacuum furnace, with a base pressure of $10^{-7}$ Torr. The furnace provides a chemically inert ambient for the sensor, enabling measurements in both vacuum and inert gas 
environments. In this work, the furnace was filled with helium to promote heat loss from the sample and thus maximize cooling rates. Measurements with cooling rates greater than $10^{3} \mathrm{~K} / \mathrm{s}$ were performed at a pressure of $1 \mathrm{Atm}$ of helium, while all other scans were performed at a pressure of 700 mTorr.

All nanocalorimetry measurements were performed using a custom low-noise data acquisition system described in detail in reference [22]. Typical noise levels in the measurements were less than $0.1 \%$. DC measurements were performed by applying current profiles that were designed to carefully control the cooling rates. AC measurements were performed at an angular frequency of $3209 \mathrm{rad} / \mathrm{s}$ using $A C$ and $D C$ current components in accordance with the selection criteria in reference [22]. For the DC measurements, both the temperature of the sample and the power supplied to it were determined directly from the sensor as described earlier. For the AC measurements, the in-phase and out-of-phase response of the sample was determined by dividing measured voltage and current signals into segments consisting of an integer number of AC oscillation periods, and applying a discrete Fourier transform (DFT) to every segment as described elsewhere $[22,26]$.

The Bi sample was prepared by means of thermal evaporation in a vacuum chamber with a base pressure of $6 \times 10^{-7}$ Torr. A $200 \mathrm{~nm}$ film of Bi was deposited directly onto the silicon nitride surface of a nanocalorimetry sensor by thermal evaporation from a $99.999 \%$ 
pure Bi source. Immediately after deposition, the Bi film was coated with a $30 \mathrm{~nm}$ layer of silicon nitride by RF sputtering. During initial melting, the Bi film broke up into a stable dispersion of small, individual islands, as expected for a liquid film that does not wet the substrate [26]. Optical micrographs of the sample (Figure 4.2a) were analyzed using digital image processing tools to yield the size distribution of the droplets (Figure $4.2 \mathrm{~b}$ ), which was well represented by a Gaussian. The total number of droplets in the sample was estimated at 6600 over an area of $1.92 \mathrm{~mm}^{2}$; the average radius of the droplets was approximately $2.0 \mu \mathrm{m}$. During melting, the silicon nitride capping layer remained on top of the Bi droplets and aided with the formation of a uniform dispersion. Experiments without silicon nitride resulted in the formation of a small number of much larger droplets. Note that the size of the droplets is not small enough to cause any significant melting point depression [82]. 


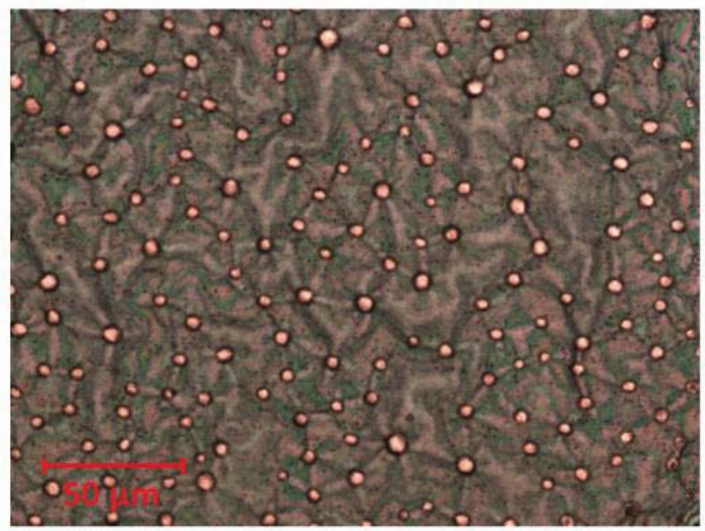

(a)

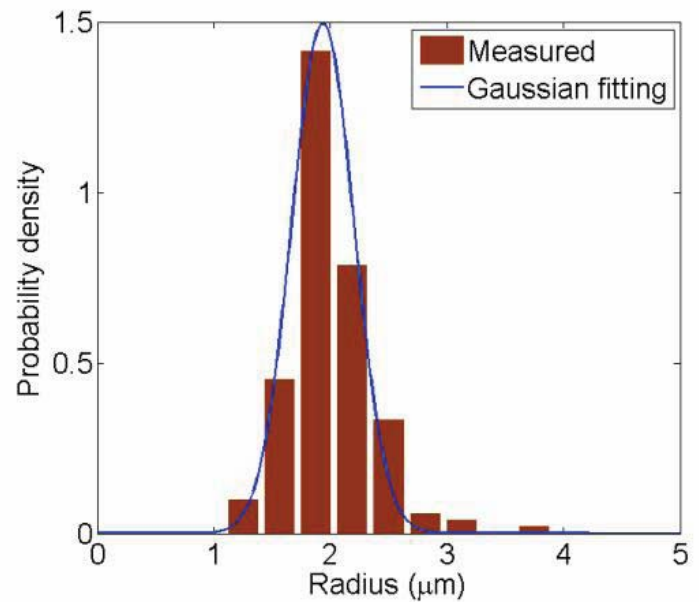

(b)

Figure 4.2. a) Optical micrograph of Bi thin film after initial melting; b) Bi islands size distribution.

\subsection{Analysis of reaction kinetics by combining DC and scanning AC measurement results}

\section{A. DC calorimetry measurements}

For a single-step reaction, it is common to assume that the rate of reaction follows [83]

$$
\frac{\dot{H}}{Q}=\frac{d x}{d t}=k(T) r(x),
$$


where $\dot{H}$ is the enthalpy flow associated with the reaction, $Q$ is the total enthalpy of reaction, and $x$ is the extent of conversion ranging from 0 to 1 . The enthalpy flow $\dot{H}$ is taken positive for an exothermic process. For a DC calorimetry measurement, the energy balance for the sensor can be written as

$$
P+\dot{H}=C \frac{d T}{d t}+L,
$$

where $P$ is the input power, $C$ is the total heat capacity of sample and addendum, and $L$ is the rate of heat loss to the environment. The power and temperature can be obtained directly from the sensor as described earlier; $C$ and $L$ can be determined as a function of temperature from a series of measurements at different cooling rates employing the procedure described in Appendix C. Once these quantities are known, the enthalpy flow $\dot{H}$ can be calculated as a function of temperature using Eq. (4.2). The conversion fraction $x$ at an instance of time $t$ is then determined as the fraction of the total enthalpy of reaction released at that time [83], given by

$$
x(t)=\frac{\int_{0}^{t} \dot{H} d t}{Q}=\frac{\int_{0}^{t} \dot{H} d t}{\int_{0}^{+\infty} \dot{H} d t} .
$$

\section{B. Scanning AC calorimetry measurements}

For a scanning AC measurement, the power supplied to the calorimeter consists of a

DC component and an AC component with angular frequency $\omega$. The resulting 
temperature response can be written as

$$
T=T_{d}+\theta,
$$

where $T_{d}(\mathrm{t})$ is the average temperature over one oscillation period and $\vartheta(t)$ represents the oscillating part of the temperature. The energy balance for the sensor can then be written as [22]:

$$
P_{d}+P_{\omega}+\dot{H}\left(T_{d}+\theta, x\right)=C \frac{d\left(T_{d}+\theta\right)}{d t}+L\left(T_{d}+\theta\right),
$$

where $P_{d}$ and $P_{\omega}$ represent the DC and AC components of the input power, respectively. If $\vartheta$ is small and the angular frequency is large enough that the change in $\mathrm{x}$ during a single oscillation period is negligible, we can linearize $L$ and $\dot{H}$ within one oscillation period so that

$$
\begin{aligned}
& L\left(T_{d}+\theta\right)=L_{d}+\left.\frac{\partial L}{\partial T}\right|_{T_{d}} \theta=L_{d}+\alpha \theta, \\
& \dot{H}=\dot{H}_{d}+\left.\frac{\partial \dot{H}}{\partial T}\right|_{T_{d}} \theta=\dot{H}_{d}+\alpha_{H} \theta,
\end{aligned}
$$

where $L_{d}$ and $\dot{H}_{d}$ are the rate of heat loss and the enthalpy flow for the nucleation process at temperature $T_{d}$, respectively. Substituting Eqs. (4.6) and (4.7) into Eq. (4.5) and separating the $\mathrm{DC}$ and $\mathrm{AC}$ terms, leads to the following two equations

$$
\begin{aligned}
& P_{d}=C \frac{d T_{d}}{d t}+\left(L_{d}-\dot{H}_{d}\right)=C \frac{d T_{d}}{d t}+L^{\prime}, \\
& P_{\omega}=C \frac{d \theta}{d t}+\left(\alpha-\alpha_{H}\right) \theta=C \frac{d \theta}{d t}+\alpha^{\prime} \theta .
\end{aligned}
$$

Both the heat capacity $C$ and the coefficient $\alpha^{\prime}$ can be determined from experimental calorimetry data using established methods $[22,26]$. In Eq. (4.9), $\alpha_{H}=-\left(\alpha^{\prime}-\alpha\right)$ is non-zero 
only during the nucleation process. Thus $\alpha_{H}$ can be obtained by first performing a least square fit of the $\alpha^{\prime}$ signal as a function of temperature in the region outside the reaction to determine the baseline, and then subtracting the baseline from the $\alpha^{\prime}$ curve. The quantity $\alpha_{H}$ is of interest because it is directly related to the kinetics of the reaction. Indeed, substituting Eq. (4.1) into Eq. (4.7) leads to the following expressions for $\alpha_{H}$

$$
\alpha_{H}=\frac{\partial \dot{H}}{\partial T}=Q \frac{\partial k(T)}{\partial T} r(x) .
$$

It is evident from this equation that

$$
\frac{\alpha_{H}}{\partial \ln k(T) / \partial T}=Q k(T) r(x)=\dot{H},
$$

i.e., the quantity on the left hand side of this equation is equal to the enthalpy flow.

Depending on the precise form of $k(T)$, this expression may be used in Eq. (4.3) to determine the extent of reaction during an $\mathrm{AC}$ measurement.

\section{Combining both $\mathrm{DC}$ and $\mathrm{AC}$ measurement results}

If we take the natural logarithm of Eqs. (4.1) and (4.11), we obtain expressions for $D C$ and $A C$ measurements

$$
\begin{aligned}
& \ln \dot{H}=\ln Q+\ln k(T)+\ln r(x), \\
& \ln \alpha_{H}-\ln \left(\frac{\partial \ln k(T)}{\partial T}\right)=\ln Q+\ln k(T)+\ln r(x) .
\end{aligned}
$$

Equations (4.12) and (4.13) can be applied to a series of DC and AC measurements 
with varying scanning rate for the analysis of reaction kinetics. For instance, for an Arrhenius type of reaction, we have:

$$
k(T)=A \exp \left(\frac{-E_{a}}{k_{B} T}\right),
$$

where $A$ is the pre-factor, $E_{\mathrm{a}}$ is the activation energy, and $k_{\mathrm{B}}$ the Boltzmann constant. Substituting Eq. (4.14) into (4.12) and (4.13) yields

$$
\begin{aligned}
& \ln \dot{H}=\ln (A Q)+\ln r(x)-\frac{E_{a}}{k_{B}} \frac{1}{T} \\
& \ln \left(\alpha_{H} T^{2}\right)-\ln \left(\frac{E_{a}}{k_{B}}\right)=\ln (A Q)+\ln r(x)-\frac{E_{a}}{k_{B}} \frac{1}{T}
\end{aligned}
$$

If the pre-factor is temperature independent, the first term on the right hand side of Eq. (4.15) is constant. If the enthalpy flow is evaluated at a fixed conversion fraction, the second term is constant also. Equation (4.15) then shows that the logarithm of the enthalpy flow is linearly related to $1 / T$. Thus a graph of the enthalpy flow at a fixed conversion fraction as a function of $1 / T$ yields a straight line with $E_{a} / k_{B}$ as slope. Equation (4.16) is a special case of Eq. (4.11) and shows that $\alpha_{H} T^{2}$ is proportional to the enthalpy flow. The conversion fraction for the $\mathrm{AC}$ measurement can then be calculated from Eq. (4.3) where $\alpha_{H} T^{2}$ takes the place of $\dot{H}$ as the integrand. Similar to Eq. (4.15), Eq. (4.16) provides a linear relationship between $\ln \left(\alpha_{H} T^{2}\right)$ and $1 / T$ with the same slope $E_{a} / k_{B}$, as long as $\alpha_{H} T^{2}$ is evaluated at a fixed conversion fraction. Since the right hand sides of Eqs. (4.15) and (4.16) are identical, it is possible to combine data points obtained from 
both $D C$ and $A C$ measurements into one linear fitting, provided that the $\ln \left(\alpha_{H} T^{2}\right)$ data points are shifted by $-\ln \left(E_{a} / k_{B}\right)$. As the parameter $E_{a}$ is not known a priori, it is necessary to perform the fit using a recursive approach.

The combination of $D C$ and $A C$ results enables kinetic analysis of the reaction over a wide range of scanning rates. It should be noted that neither Eq. (4.12) nor (4.13) requires a constant scanning rate, but rather the direct measurements of $\dot{H}$ and $\alpha_{H}$. This is an especially useful property for nano-calorimetric measurements, where the latent heat can easily change the scanning rate, and a feedback control of the temperature is not always feasible. We will show in the next section that the same technique can also be applied to non-Arrhenius type processes such as nucleation.

\subsection{Application to nucleation theory}

According to classic nucleation theory, the nucleation rate for a spherical nucleus is given as a function of temperature by the following expression [70]:

$$
J=A \exp \left[-\Delta G^{*} f(\theta) / k_{\mathrm{B}} T\right],
$$

where the activation barrier to nucleation $\Delta G^{*}$ is given by

$$
\Delta G^{*}=\frac{16 \pi}{3} \frac{\gamma_{\mathrm{SL}}^{3} T_{\mathrm{m}}^{2}}{L_{\mathrm{v}}^{2}} \frac{1}{\Delta T^{2}},
$$

and where $k_{\mathrm{B}}$ denotes the Boltzmann constant, $\gamma_{\mathrm{SL}}$ is the solid-liquid interfacial energy, $T_{\mathrm{m}}$ is the melting temperature, $\Delta T=T_{\mathrm{m}}-T$ is the degree of undercooling, and $L_{\mathrm{v}}$ is the 
enthalpy of melting. $A$ is a kinetic pre-factor that depends exponentially on temperature [73]. The temperature dependence of the pre-factor is, however, much weaker than that of the exponential factor in Eq. (4.17), because of the strong dependence of $\Delta G^{*}$ on the undercooling. Consequently, $A$ is treated as practically insensitive to temperature in the analysis of nucleation kinetics $[63,64,70,75]$. The function $f(\vartheta)$ is the catalytic potency factor for heterogeneous nucleation and is defined as the ratio of the activation energies for heterogeneous and homogeneous nucleation, respectively. This factor can be written in terms of the contact angle $\vartheta$ of the nucleus,

$$
f(\theta)=(2+\cos \theta)(1-\cos \theta)^{2} / 4,
$$

and is equal to unity for an angle of $\pi$. If the size of the droplets in the dispersion is small $(<10 \mu \mathrm{m})$, as is the case in our study, the majority of the droplets should be free from catalytic nucleation sites within their volume $[72,77,84]$. Thus nucleation will either proceed homogeneously in the bulk of the droplets or be catalyzed by the interface with the silicon nitride. In the latter case, we assume that all droplets have the same contact angle $\vartheta$, and thus the same catalytic potency factor $f(\vartheta)$. The rate-determining step in the solidification of a very small metal droplet is the formation of a nucleus because the time to form a critical nucleus is typically much longer than the time it takes the nucleus to consume the droplet [85]. Consequently, we assume that a droplet solidifies instantly upon formation of a nucleus. If we assign all droplets with volume $v_{i}$ (and thus a surface 
or interfacial area of $s_{i}$ ) in the distribution to group $i$, and if we denote the number of droplets and the nucleation frequency per droplet in that group by $n_{i}$ and $I_{i}$, respectively, the rate of nucleation then follows the law of radioactive decay [70]

$$
\frac{d n_{i}}{d t}=-n_{i} I_{i},
$$

provided the number of droplets is sufficiently large. For bulk nucleation, the expected nucleation frequency is given by

$$
I_{i}=J v_{i},
$$

while for interface nucleation:

$$
I_{i}=J_{S_{i}} .
$$

Integrating Eq. (4.20) over time, it then follows that

$$
n_{i}=n_{i 0} \exp \left(-\int_{0}^{t} I_{i}(T(t)) d t\right),
$$

where $n_{i 0}$ is the initial number of droplets in group $i$, and where the nucleation frequency $I_{i}$ varies with time via the temperature history of the sample. The total volume of un-solidified droplets and the volumetric rate of solidification can then be calculated as

$$
\begin{aligned}
& V=\sum_{i=1}^{m} n_{i} v_{i}=\sum_{i=1}^{m} v_{i} n_{i 0} \exp \left(-\int_{0}^{t} I_{i} d t\right), \\
& \frac{d V}{d t}=\sum_{i=1}^{m} \frac{d n_{i}}{d t} v_{i}=-\sum_{i=1}^{m} n_{i 0} v_{i} I_{i} \exp \left(-\int_{0}^{t} I_{i} d t\right),
\end{aligned}
$$

where $m$ is the total number of groups in the distribution and $V$ is the total volume of 
droplets at a given time. Equations (4.24) and (4.25) are very general and hold for any temperature history, but they are not very useful in the analysis of experimental data because it is difficult to resolve the nucleation frequency for islands of different size. If the size distribution is sufficiently tight, however, the equations can be reformulated in terms of the average volume or interfacial area of the droplets,

$$
\begin{aligned}
& V=\left(\sum_{i=1}^{m} n_{i 0} v\right) \exp \left(-\int_{0}^{t} I d t\right)=V_{0} \exp \left(-\int_{0}^{t} I d t\right), \\
& \frac{d V}{d t}=-\left(\sum_{i=1}^{m} n_{i 0} v\right) I \exp \left(-\int_{0}^{t} I d t\right)=-I V_{0} \exp \left(-\int_{0}^{t} I d t\right),
\end{aligned}
$$

where $V_{0}$ is the initial total volume of all liquid droplets and $I$ is the nucleation frequency determined from the average volume $v$ or interfacial area $s$ of the droplets, depending on whether the nucleation process occurs in the bulk or at the interface. In Appendix $D$, we analyze the error associated with this simplification and demonstrate that this approach works well for the distribution of droplet sizes in this study. Equation (4.27) provides the volumetric rate of solidification of a sample, which is directly related to the instantaneous enthalpy flow $\dot{H}$ during solidification

$$
\dot{H}=-L_{v} \frac{d V}{d t}=Q I \frac{V}{V_{0}}=Q I(1-x)
$$

where $Q=L_{v} V_{0}$ is the total enthalpy released during solidification and the conversion fraction is given by $x=1-V / V_{0}$. Equation (4.28) shows that the solidification of a dispersion of droplets can be described using a deterministic rule, even though individual 
nucleation events are stochastic. Comparing Eq. (4.28) with Eq. (4.1), the nucleation model can be cast into the general formalism by letting

$$
\begin{aligned}
& k(T)=I=A^{*} \exp \left(-\frac{B}{T \Delta T^{2}}\right), \\
& r(x)=1-x,
\end{aligned}
$$

where $A^{*}=A v$ or $A s$ for bulk or interface nucleation, respectively, and $B$ is the kinetic factor

$$
B=\frac{16 \pi \gamma_{\mathrm{SL}}^{3} T_{\mathrm{m}}^{2}}{3 L_{\mathrm{v}}^{2} k_{\mathrm{B}}} f(\theta) .
$$

Substituting Eqs. (4.29) and (4.30) into Eqs. (4.12) and (4.13) yields the following equations for $D C$ and $A C$ measurements

$$
\begin{aligned}
& \ln I=\ln \dot{H}-\ln [(1-x) Q]=\ln A^{*}-\frac{B}{T \Delta T^{2}}, \\
& \ln I=\ln \left(\frac{\alpha_{H}}{g}\right)-\ln [(1-x) Q]-\ln B=\ln A^{*}-\frac{B}{T \Delta T^{2}},
\end{aligned}
$$

where

$$
g=\frac{(\Delta T-2 T)}{T^{2} \Delta T^{3}} .
$$

For a given conversion fraction, both (1-x) and $Q$ are known constants. Thus, Eq. (4.32) provides a way to calculate the nucleation frequency I from the enthalpy flow in a DC measurement. Equation (4.32) also shows that the logarithm of the enthalpy flow is linearly related to $1 / T \Delta T^{2}$, with the kinetic factor $B$ as the slope. Similar to Eq. (4.32), Eq. (4.33) provides a linear relationship between $\ln \left(\alpha_{H} / g\right)$ and $1 / T \Delta T^{2}$ with the same slope $B$, 
as long as $\alpha_{H} / g$ is evaluated at the same fixed conversion fraction. The conversion rate can be calculated from Eq. (4.3), where $\alpha_{H} / g$ replaces $\dot{H}$ as the integrand. As the right hand sides of Eqs. (4.32) and (4.33) are identical, it is possible to combine data points obtained from both DC and AC measurements into one linear fit, provided that the $\ln \left(\alpha_{H-}\right.$ /g) data points are shifted by $-\ln B$, which can be done with a recursive approach.

\subsection{Results}

Figure 4.3a displays cooling rate traces for a total of 22 scans of the Bi sample. The solid curves represent DC scans with cooling rates ranging from $\sim 2 \times 10^{4} \mathrm{~K} / \mathrm{s}$ to $\sim 1 \times 10^{3} \mathrm{~K} / \mathrm{s}$, while the dashed curves represent $A C$ scans with cooling rates from $\sim 1 \times 10^{3} \mathrm{~K} / \mathrm{s}$ to $\sim 30$ $\mathrm{K} / \mathrm{s}$. During solidification, the cooling rate first decreases as the latent heat is released. This drop is followed by an increase in cooling rate, sometimes overshooting the original cooling rate, especially at smaller cooling rates. This overshooting is not the result of any phase transition, but is caused by a temporary increase in heat loss from the sample as discussed elsewhere $[22,26]$. It is evident from the figure that solidification takes place at ever-lower temperatures as the cooling rate increases.

Figure $4.3 \mathrm{~b}$ shows ten DC cooling scans obtained under identical experimental conditions. It is evident that the cooling trace is extremely reproducible. The solidification process starts at approximately $450 \mathrm{~K}$ and ends around $400 \mathrm{~K}$, spread out 
over more than $50 \mathrm{~K}$. This result stands in contrast with experiments on single droplets, where the stochastic nature of the nucleation event causes the trace to vary significantly from measurement to measurement. This reproducibility is in line with previous measurements on similar samples $[22,26]$ and provides direct evidence that the number of isolated Bi droplets is sufficiently large that Eq. (4.20) is valid.

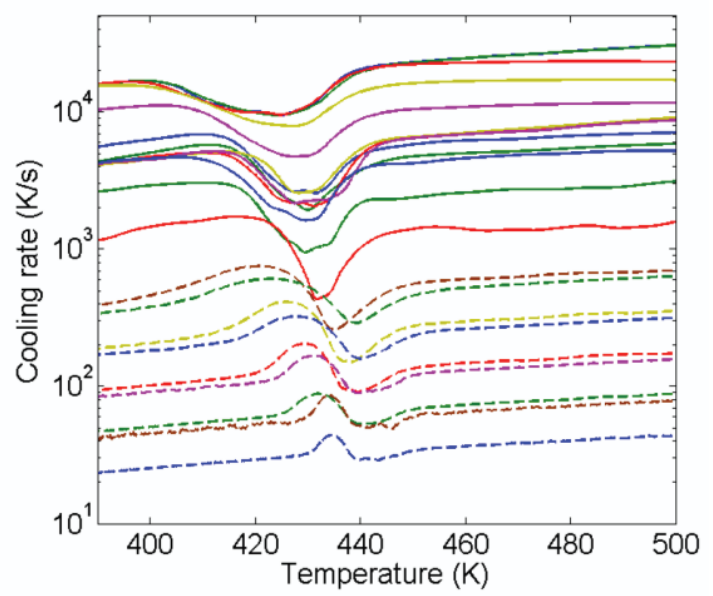

(a)

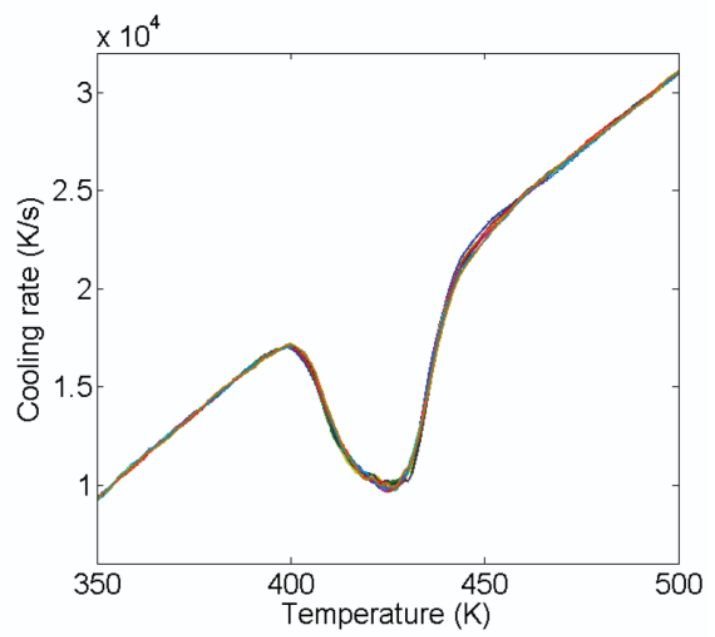

(b)

Figure 4.3. a) Cooling rate curves for 13 DC scans (solid curves) and 9 AC scans (dashed curves); b) Cooling rate curves for $10 \mathrm{DC}$ scans performed under identical conditions.

Using the energy balance, Eq. (4.2), the enthalpy flow $\dot{H}$ can be determined as a 
function of time from the DC data in Figure 4.3a. The results are shown in Figure 4.4a. As the cooling rate decreases, the maximum amplitude of enthalpy peak decreases and the solidification process takes a longer period of time to complete. The same trends appear in the $\alpha_{H} / g$ curves for the AC scans (Figure 4.4b), which is expected given that $\alpha_{H} / g$ is proportional to $\dot{H}$ according to Eqs. (4.32) and (4.33). The duration of the solidification process changes from several milliseconds for the fastest DC scan to several seconds for the slowest AC scan. In both cases, we calculate the nucleation frequency at $50 \%$ conversion to minimize any errors introduced by the baseline subtraction, using either Eq. (4.32) or Eq. (4.33). 


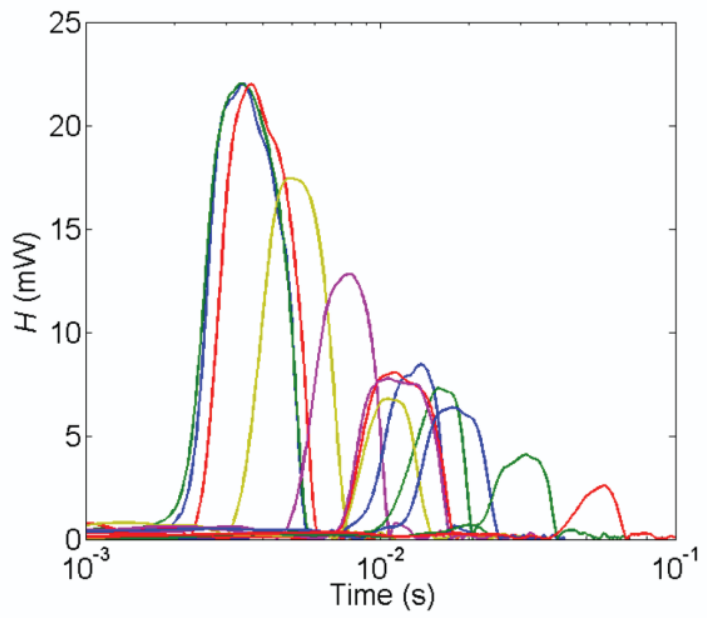

(a)

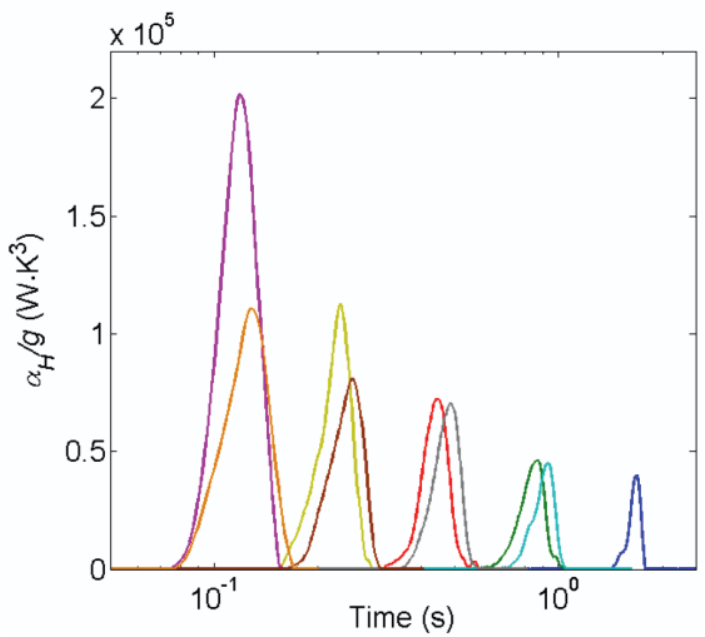

(b)

Figure 4.4. a) Reaction enthalpy flow as function of time for nucleation in DC cooling scans. b) $\alpha H / g$ curves as a function of time for nucleation in $\mathrm{AC}$ cooling scans. The origin of time corresponds to $500 \mathrm{~K}$ for each cooling scan, and the corresponding cooling rates are shown in Figure 4.3a.

Figure 4.5 a shows the undercooling at $50 \%$ conversion as a function of cooling rate, where the cooling rate at $450 \mathrm{~K}$ was used as the nominal cooling rate. As expected, larger cooling rates result in greater undercooling. The maximum undercooling exceeds $120 \mathrm{~K}$ at cooling rates greater than $2 \times 10^{4} \mathrm{~K} / \mathrm{s}$, while a cooling rate of $30 \mathrm{~K} / \mathrm{s}$ still results in an undercooling larger than $100 \mathrm{~K}$. Figure $4.5(\mathrm{~b})$ shows a combined plot of $\ln (/)$ for all DC 
and $A C$ cooling scans as a function of $1 / T \Delta T^{2}$. In this graph, the AC data were shifted in the $y$-direction by an amount $-\ln (B)$ as seen in Eq. (4.33), where $B$ was determined using recursive least squares regression. The figure clearly illustrates that the $A C$ and $D C$ data are in good agreement with each other and in particular that the slopes of both sets of data are the same. The linear relationship between the data indicates that a single nucleation mechanism is active over three orders of magnitude of cooling rate. The kinetic factor $B$ and the pre-factor $A^{*}$ can be calculated from the slope and intercept of the linear fit using Eq. (4.32) or (4.33). The catalytic potency factor $f(\vartheta)$ and the contact angle can be calculated from Eqs. (4.31) and (4.19) using literature values for the physical properties of Bi: $T_{\mathrm{m}}=544.7 \mathrm{~K}, L_{\mathrm{v}}=5.288 \times 10^{8} \mathrm{~J} / \mathrm{m}^{3}$, and $\gamma_{\mathrm{SL}}=0.0544 \mathrm{~J} / \mathrm{m}^{2}$ [73]. The results are summarized in Table 4.1. In the analysis, the interfacial area was calculated as the total surface area of the droplets because the droplets were completely encapsulated in silicon nitride. 


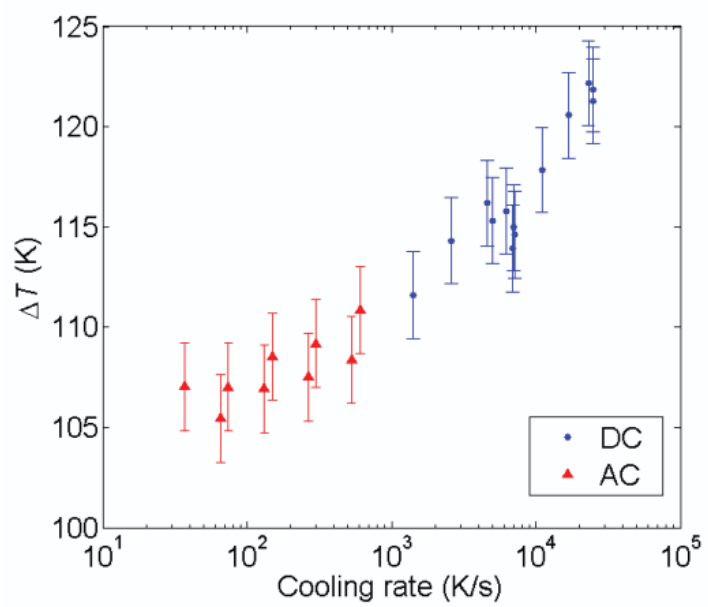

(a)

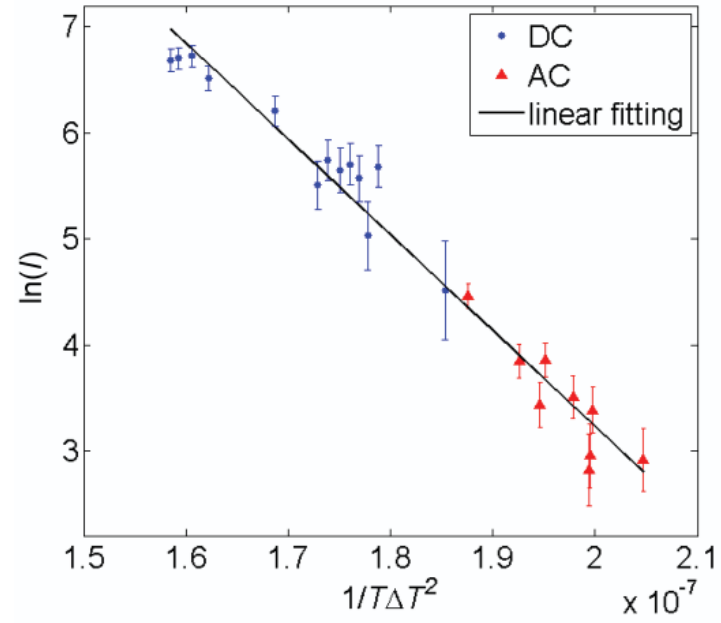

(b)

Figure 4.5. a) Undercooling at 50\% conversion as a function of the nominal cooling rate at $450 \mathrm{~K}$. b) Graph of the logarithm of the nucleation frequency at $50 \%$ conversion as a function of $1 / T \Delta T 2$.

Table 4.1. Summary of results

\begin{tabular}{|c|c|c|c|c|c|c|}
\hline$Q(\mathrm{~J})$ & $\begin{array}{l}\text { Intercept } \\
\left(\ln \left(s^{-1}\right)\right)\end{array}$ & $A^{*}\left(\mathrm{~s}^{-1}\right)$ & $\begin{array}{l}A=A^{*} / \mathrm{s} \\
\left(\mathrm{m}^{-2} \mathrm{~s}^{-1}\right)\end{array}$ & $B\left(\mathrm{~K}^{3}\right)$ & $f(\theta)$ & $\vartheta\left(^{0}\right)$ \\
\hline $\begin{array}{c}(5.15 \pm \\
0.27) \times 10^{-5}\end{array}$ & $\begin{array}{c}21.31 \pm \\
1.4\end{array}$ & $\begin{array}{c}1.8 \times \\
10^{(9 \pm 0.61)}\end{array}$ & $\begin{array}{c}3.58 \times \\
10^{(19 \pm 0.61)}\end{array}$ & $\begin{array}{c}(9.04 \pm 0.76) \\
\times 10^{7}\end{array}$ & 0.436 & 85.1 \\
\hline
\end{tabular}




\subsection{Discussion}

Nucleation of a solid phase in a melt is by nature a stochastic process. In the present case, the solidification peaks of a thin-film sample of $\mathrm{Bi}$ are highly reproducible, giving the appearance of a deterministic solidification process. The reproducibility in our experiments arises because the Bi film breaks up into a very large number of isolated islands and solidification of the sample is not the result of a single nucleation event, but that of a very large number of independent events. The calorimetry trace can be regarded as the cumulative superposition of the traces for all individual nucleation events and there is no need to perform a large number of measurements at a given cooling rate.

The nanocalorimetry measurements demonstrate significant undercooling before nucleation takes place, but the degree of undercooling varies less than $20 \mathrm{~K}$ as the cooling rate is changed over three orders of magnitude. This result stands in contrast with typical chemical reactions, where changes in heating rate result in much larger shifts in reaction temperature [83]. Thus the ability to study nucleation over a wide range of cooling rates is essential for accurate measurement of the kinetic parameters. Scanning DC nanocalorimetry can be used to study the reaction at ultra-fast cooling rates, while scanning AC calorimetry works for medium and low rates. The maximum cooling rate is set by the heat loss from the sample to the environment. Considering the 
large interface to volume ratio of the thin-film sample, this heat loss occurs primarily by heat transport through the helium, and can be enhanced by increasing the pressure and by decreasing the distance between the sample and a nearby heat sink. The minimum cooling rate is set by the resolution of the $\alpha$-signal in the AC measurements. As the cooling rate decreases, the peak in the $\alpha$-curve decreases, resulting in a larger relative error following baseline subtraction. This constraint can be relaxed by amplifying the $\alpha$-signal using a lock-in amplifier. The technique described in Sec. 4.3 makes it possible to combine both $\mathrm{DC}$ and $\mathrm{AC}$ measurement results in the analysis of reaction kinetics. The technique can be applied to general single-step reactions, and depending on the specific reaction model, may require a recursive approach in order to combine both $D C$ and $A C$ data points into one single curve. This approach is valid only if the reaction of interest follows the same reaction model over the entire range of scanning rates.

Equations (4.32) and (4.33) are derived through a straightforward application of classical nucleation theory to a collection of droplets. The equations provide a convenient means of analyzing nucleation results as illustrated by the results for $\mathrm{Bi}$ in Figure 4.5b. The straight line in the $\log (I)$ vs $1 / T \Delta T^{2}$ plot demonstrates that the nucleation rate is well described by classical nucleation theory. It also indicates a single nucleation mechanism is active over three orders of magnitude of cooling rate [64]. As noted before, the droplets are too small for bulk heterogeneous nucleation [77, 84], 
leaving the possibilities of either homogenous nucleation or heterogeneous nucleation catalyzed by the interface with the silicon nitride. This is further supported by the fact that nucleation does not usually follow the classical rate equation (4.17) if bulk heterogeneous nucleation is dominant, because accidental catalysts of varying degrees of effectiveness (i.e. different $f(\vartheta) \quad$ values) are liable to be present in different droplets [73]. The value of $f(\vartheta)$ is found to be less than unity, indicating that the $\mathrm{Bi} / \mathrm{SiN}_{\mathrm{x}}$ interface catalyzes the nucleation process. Considering the large interface-volume ratio for the droplets, it is not surprising that interface heterogeneous nucleation is preferred. We further note that the value of $A$ in our measurement is much smaller than the theoretical estimate of $10^{29 \pm 1} \mathrm{~m}^{-2} \mathrm{~s}^{-1}$ by Turnbull [73]. Such a difference is not uncommon and can be accounted for by a modest temperature-dependence of the interfacial energy, to which $A$ is extremely sensitive $[70,86]$. For example, Turnbull measured a value of $A$ that is 7 orders of magnitude larger than the theoretical estimate, which can be accounted for by a temperature-dependence of approximately $0.1 \% / \mathrm{K}$ of the interfacial energy [70]. In fact, Spaepen has exploited this discrepancy between the experimental and theoretical values of $A$ to study the temperature-dependence of the interfacial energy [86].

The Bi nucleation behavior we observed is similar to a previous study by Chaubey et al. [78] on the nucleation of $\mathrm{Bi}$ droplets entrapped in a $\mathrm{Zn}$-matrix, where the main 
nucleation mechanism was found to be interface heterogeneous nucleation catalyzed by the interface with the solid $\mathrm{Zn}$. This is to be expected as the droplet size in their study averaged around $6 \mathrm{~nm}$, making the presence of catalytic sites inside the droplets highly unlikely. The cooling rate used by Chaubey's was $2.5 \mathrm{~K} / \mathrm{min}$, resulting in an undercooling of $132 \mathrm{~K}$. This undercooling is larger than the undercooling observed in this study and can be attributed to the smaller droplet size and the less catalytic interface with solid $\mathrm{Zn}$. Perepezko $[72,87]$ has reported an even larger undercooling of $227 \mathrm{~K}$ in a study of $\mathrm{Bi}$ dispersions with a 10 to $20 \mu \mathrm{m}$ droplet size. The Bi droplets were suspended in a polyphenyl ether carrier fluid and maintained independent through use of thin surface coatings produced by a chemical reaction [71]. The large undercooling indicates that the surface coatings used in his study are even less catalytic. Our observation stands in contrast with measurements over a wide range of cooling rates on a single $10 \mu \mathrm{m}$ droplet of tin by Yang et al [60], where nanocalorimetry uncovered two different nucleation mechanisms, i.e., interface and bulk heterogeneous nucleation.

Finally we need to point out that there are two experimental factors that could potentially affect the results adversely: the temperature uniformity across the sample area and the temperature oscillations of the $A C$ measurements. We have performed extensive finite element simulations of the temperature distributions in the nano-calorimetry sensor [22]. These simulations show that the temperature distribution 
is quite uniform across the sample area with $\sim 2 \%$ of variation and that it is nearly identical for all scans [22]. Thus the effect of temperature non-uniformity on the nucleation analysis is negligible. The scanning AC measurements were conducted following protocols established previously [22] and the corresponding temperature oscillation were controlled to be $\sim 3 \mathrm{~K}$ at the solidification temperature. This temperature oscillation is small compared to the undercooling so that the linearization in Eq. (4.6) and (4.7) is acceptable.

\subsection{Summary}

We have studied the nucleation behavior of Bi droplets over a wide range of cooling rates by combining both scanning $\mathrm{DC}$ and $\mathrm{AC}$ nano-calorimetry techniques. The $\mathrm{Bi}$ sample is fabricated using thermal evaporation and encapsulated in silicon nitride. Upon initial melting, the Bi film breaks up into a large number of individual droplets that solidify independently. As a result the calorimetry trace samples a very large number of nucleation events resulting in highly repeatable measurements in spite of the stochastic nature of nucleation. By applying classical nucleation theory to a collection of droplets under the conditions of a DC or AC scanning calorimetry experiment, simple equations are derived to analyze the calorimetry traces for a wide range of cooling rates. The results indicate that nucleation of solid Bi occurs heterogeneously catalyzed by the 
interface with the silicon nitride and that a single nucleation mechanism is active over three orders of magnitude of cooling rate. 


\section{Chapter 5}

\section{Kinetics of solid-gas reactions characterized by scanning AC nano-calorimetry with application to Zr oxidation ${ }^{*}$}

\subsection{Introduction}

Reactions between solids and gasses play an important role in modern technology [88]. They encompass a broad field, including oxidation of metals, reduction of oxides, roasting of sulfide minerals, synthesis of thin-film coatings, and catalysis, to cite just a few examples. State-of-the-art integrated circuits would be unthinkable without the oxidation reaction to create the gate oxide or the etch processes to define patterns. Use of structural materials at elevated temperatures is often limited by their oxidation behavior, but can be extended through use of protective coatings [89-91].

Solid-gas reactions, and high-temperature oxidation reactions in particular, are often characterized using thermogravimetric analysis (TGA). In this technique changes in the mass of the solid are measured as a function of temperature and time, and related to the extent of reaction. The technique works very well for bulk materials, but is less effective for coatings because the coating needs to be removed from the substrate to

\footnotetext{
${ }^{*}$ Reprinted with permission from K. Xiao, D. Lee and J. J. Vlassak, Applied Physics Letters 105 (17), 171901
} (2014). Copyright 2014, AIP Publishing LLC 
avoid the enormous dilution effect caused by substrate $[92,93]$. This extra step in sample preparation can be problematic and is sometimes not feasible when the sample is very thin [92].

Here we propose the use of nano-calorimetry to characterize the oxidation behavior of very thin coatings. Nano-calorimetry is a very sensitive calorimetry technique capable of measuring the thermodynamic properties of nano-quantities of materials. The technique has been applied to study phase transformations in thin-film samples with thickness as small as a few nanometers $[5,16,54,55,61,82,94-96]$. A recently developed scanning AC nano-calorimetry technique can be used to measure heat capacity over a wide range of temperatures (up to $1300 \mathrm{~K}$ ) and heating rates (from isothermal to $3000 \mathrm{~K} / \mathrm{s})[22,24,81]$. The technique has been combined with in-situ X-ray diffraction for structural analysis of reaction products [26, 97]. Here we describe how the technique can be used to study solid-gas phase reactions by measuring the change in heat capacity of a sample during reaction. Unlike traditional DC techniques, the scanning $A C$ technique is insensitive to heat loss from the sample to the environment [22]. This makes the technique ideal for analyzing solid-gas phase reactions, where the heat loss is significant compared to the reaction heat flow. We demonstrate this approach by evaluating the oxidation kinetics of sputter-deposited thin films of zirconium in air. 


\subsection{Experimental details and results}

The nanocalorimetry measurements in this study were performed using a parallel nano-scanning calorimeter (PnSC) device. This device is described in detail elsewhere [7, $22,23]$. Briefly, it consists of a silicon substrate with a $5 \times 5$ array of independently controlled nano-calorimeter sensors as shown in Figure 5.1. Each sensor contains a tungsten four-point electrical probe that serves both as a heating element and a resistance thermometer and that is fully encapsulated within a silicon nitride membrane. In a typical calorimetry measurement, a thin-film sample is deposited in the shaded area between the two sensing leads and an electric current is supplied through the tungsten heating element. The measured current and voltage are used to determine the power supplied to the sample, while the temperature of the sample is determined from the resistance of the heating element, which is calibrated to temperature [22]. The PnSC is installed in a probe card that is mounted inside a high-vacuum furnace with a base pressure of $1 \times 10^{-7}$ Torr. In this study, all measurements were performed either in vacuum or in air. 


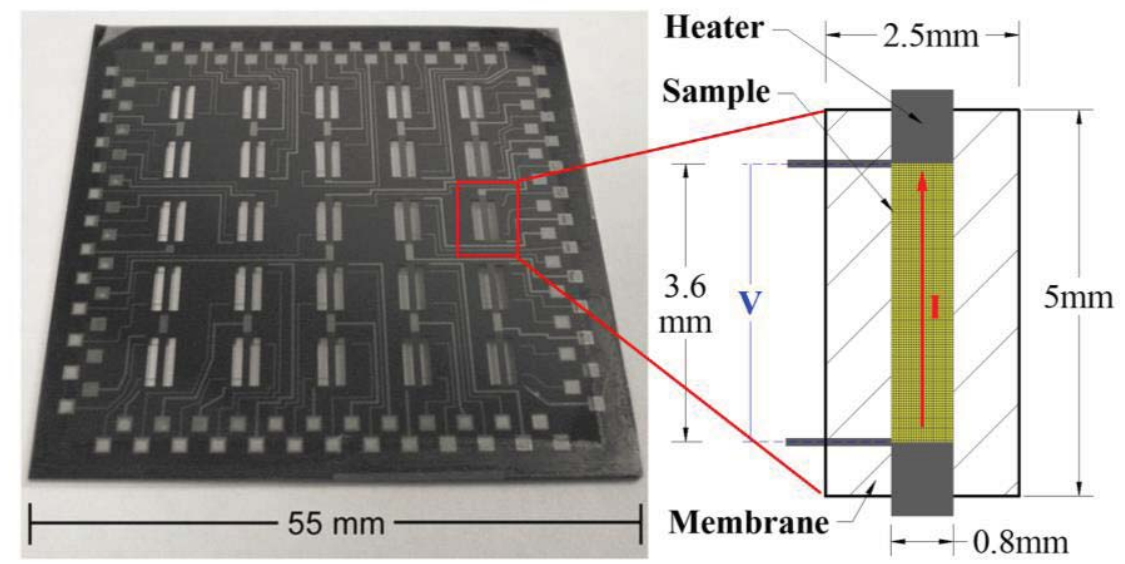

Figure 5.1. (left) photo of the PnSC device; (right) schematic of the calorimetry sensor.

All nanocalorimetry measurements were made using a custom, low-noise data acquisition system described in detail in reference $[22,26]$. A carefully designed current profile, containing direct current (DC) and alternating current (AC) components, was applied to the nano-calorimeter sensors to attain the desired temperature trajectory for isothermal or temperature scanning measurements. The AC component, at a frequency of $520.83 \mathrm{~Hz}$, produced temperature oscillations of approximately $5 \mathrm{~K}$ in the sample. As described in detail elsewhere [22], the nonlinear response of the sensors introduces harmonic components into the measured voltage, which are then isolated using harmonic analysis. By determining the amplitude $V_{2 f}$ of the $2 f$ harmonic, the heat capacity of sample and addendum can be calculated from [22]:

$$
C=\frac{5 i^{2} I_{0} R_{0} k}{8 \pi f V_{2 f}},
$$

where $I_{0}$ is the DC component of the applied current, $i$ the amplitude of the AC 
component, $R_{0}$ the ambient-temperature resistance of the calorimeter sensor, and $k$ its temperature derivative. In the harmonic analysis, the experimental sensor voltage was partitioned into segments containing two cycles and Eq. (5.1) was evaluated for each segment to provide $C$ as a function of time and temperature.

After fabrication of the PnSC device, the nano-calorimetry sensors were calibrated to determine the $R_{0}$ and $k$ values [7, 22]. Zirconium films with a thickness of approximately $200 \mathrm{~nm}$ were deposited using magnetron sputtering from a $\mathrm{Zr}$ (99.999\% pure) target in a DC magnetron deposition system (AJA International, North Scituate, MA) with a base pressure of $1 \times 10^{-7}$ Torr. The magnetron was powered at $80 \mathrm{~W}$ and the 43-minute deposition proceeded in an atmosphere of 5 mTorr of $\mathrm{Ar}$. $\mathrm{Zr}$ deposition was limited to the sample area of the calorimeter sensors through use of a shadow mask. Test samples deposited under identical conditions on dummy substrates were analyzed with profilometry to determine the film thickness $h_{z r}$.

X-ray diffraction measurements were performed on the samples before and after oxidation using a D8 Discover diffraction system (Bruker, Billerica, MA). In the as-deposited state, the Zr films had a hexagonal crystal structure with a $\{0002\}$ fiber texture, as indicated in Figure 5.2 (Powder Diffraction File \#01-078-2921 [98]). Before the oxidation experiments, the $\mathrm{Zr}$ samples were heated three times to $1150 \mathrm{~K}$ and then cooled to room temperature at a rate of $700 \mathrm{~K} / \mathrm{s}$. These heating cycles were performed 
in vacuum using the nano-calorimeter sensors and served to measure the heat capacity of the samples as a function of temperature. The heating cycles also resulted in sharper $\operatorname{Zr}\{0002\}$ diffraction peaks (Figure 5.2), as the grain size increased from $18 \mathrm{~nm}$ in as-deposited $\mathrm{Zr}$ to $33 \mathrm{~nm}$ based on a Scherrer analysis of the diffraction peaks. The annealed $\mathrm{Zr}$ samples were then oxidized by heating the nano-calorimeter sensors in air. In the XRD scans obtained after the oxidation experiments, the $\mathrm{Zr}$ diffraction peak was replaced with the $\{-111\}$ peak of monoclinic $\mathrm{ZrO}_{2}$ (Powder Diffraction File \#01-070-2491 [98]), indicating complete oxidation of the $\mathrm{Zr}$ to $\mathrm{ZrO}_{2}$.

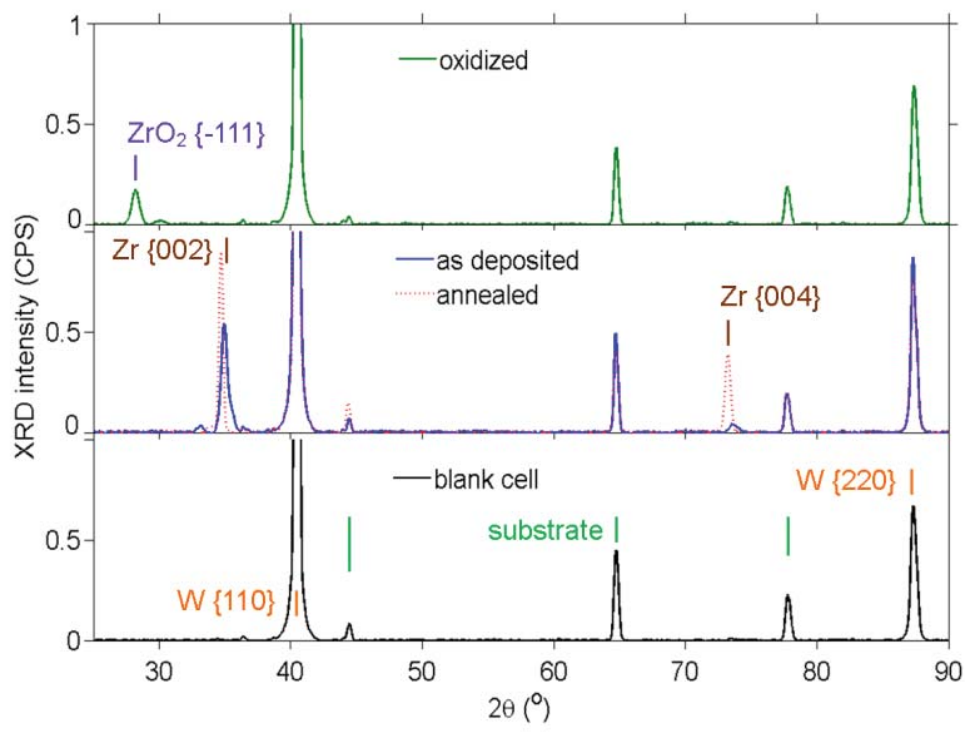

Figure 5.2. XRD patterns for cell with no sample, with Zr sample and with sample after oxidation experiments. The patterns were collected for $300 \mathrm{~s}$ each.

Isothermal oxidation experiments were performed in air on $\mathrm{Zr}$ thin-film samples, while measuring the heat capacity of the samples in situ. Because $\mathrm{ZrO}_{2}$ has a larger specific heat capacity than $\mathrm{Zr}$ [99], the heat capacities of the samples increase as the 
oxide scale grows. The increase in heat capacity of the samples, $\Delta C$, is readily measured using scanning AC nano-calorimetry. The results are plotted in Fig 5.3(a) as a function of time and for different temperatures. Test temperatures below $1200 \mathrm{~K}$ were selected to avoid phase transformations in $\mathrm{Zr}$ and $\mathrm{ZrO}_{2}$, so that the change heat capacity can be attributed solely to oxidation of the Zr. The thickness of the oxide scale is then related to the change in heat capacity by

$$
\Delta h=\frac{\Delta C}{\left(C_{m, Z r O_{2}}-C_{m, Z r}\right)} \frac{V_{m, Z r O_{2}}}{S}=\lambda \Delta C,
$$

where $\Delta h$ is the change in oxide thickness during the experiment, $V_{m, Z r O 2}$ is the molar volume of $\mathrm{ZrO}_{2}$, and $S$ represents the sample area over which the $\mathrm{Zr}$ oxidizes. In this expression, $C_{m, Z r O 2}$ and $C_{m, Z r}$, represent the molar heat capacities of $\mathrm{ZrO}_{2}$ and $\mathrm{Zr}$, respectively. If the rate of oxidation is limited by diffusion of oxygen through the oxide layer, which is expected for our experimental conditions [100,101], growth of the oxide layer is described by the following equation [100]:

$$
\frac{d \Delta h}{d t}=\frac{K}{\Delta h+h_{i}}
$$

where $K$ is the parabolic rate constant and $h_{\mathrm{i}}$ is the oxide thickness at the onset of the isothermal segment of the experiment, which consists of both the native oxide and the oxide grown during the temperature ramp that precedes the isothermal segment. $K$ is typically described by an Arrhenius relationship [101, 102] and a logarithmic graph of $K$ 
versus reciprocal temperature provides the activation energy $E_{\mathrm{a}}$ for oxygen diffusion through the oxide. During isothermal oxidation, $K$ is constant and Eq. (5.3) is readily integrated over time. Noting that the time origin is chosen at the start of the isothermal section when $\Delta h=0$, we find

$$
\Delta h^{2}+2 \Delta h h_{i}=2 K t .
$$

Equation (5.4) represents the well-known parabolic rate law for isothermal oxidation. During isothermal oxidation, $\lambda$ is constant and the oxide thickness scales linearly with the change in heat capacity of the sample. Combining Eqs. (5.2) and (5.4) then yields an equation that describes the evolution of the heat capacity of the sample during oxidation,

$$
\Delta C^{2}+2 \Delta C C_{i}=2 \frac{K}{\lambda^{2}} t=2 K_{c} t,
$$

where $C_{i}=h_{i} / \lambda$. The parameters $C_{i}$ and $K_{c}$ can be determined from a least-squares fit of Eq. (5.5) to the experimental $\Delta C$ - $t$ data in Figure5.3. The fitting results are shown as solid lines in Fig 5.3(a), and the corresponding values of $C_{i}$ and $K_{c}$ are listed in Table 5.1. Equation (5.5) agrees well with the experimental heat capacity curves up to approximately $80 \%$ of their maximum value, demonstrating that the reaction indeed follows the parabolic rate law and oxidation is diffusion limited. As most of the $Z r$ is consumed, however, the oxidation rate decreases below the parabolic rate. This is caused by the non-uniform temperature distribution in the nano-calorimeter sensors [ 
$22,23]$, which gradually lowers the average oxidation rate as the $\mathrm{Zr}$ is consumed through the thickness of the sample. The value of $\lambda$ can be calculated from the change in heat capacity of a fully oxidized sample and the corresponding $\mathrm{ZrO}_{2}$ thickness,

$$
\lambda=\frac{h_{Z r O_{2}}}{\left(C_{x}\right)_{\max }+C_{i}}=\frac{R_{P B} h_{Z r}}{\left(C_{x}\right)_{\max }+C_{i}},
$$

where $h_{\mathrm{ZrO} 2}$ is the thickness of $\mathrm{ZrO}_{2}$ film and $R_{P B}$ is the Philling-Bedworth ratio for $\mathrm{Zr}$, which is taken to be 1.56 [103]. The value of $\lambda$ is found to be $(0.76 \pm 0.05) \mathrm{m} /(\mathrm{J} / \mathrm{K})$ for all samples. This value is larger than expected based on the sample areas of the sensors, because the temperature non-uniformity within the sensing areas decreases the area over which $\mathrm{Zr}$ is oxidized. Once $\lambda$ is known, the parabolic rate constant $K$ is calculated from $K=\lambda^{2} K_{\mathrm{c}}$. A logarithmic graph of $K$ versus reciprocal temperature is shown in Figure 5.3(b) for all isothermal measurements, together with values reported in previous works $[102,104]$. The value obtained in our measurements is roughly one to two orders of magnitude larger than the literature values, which may be expected because the very small grain size of our samples promotes grain boundary diffusion of oxygen through the oxide [105]. A least square line fit of the data then yields an activation energy of $E_{a}=0.60 \pm 0.09 \mathrm{eV}$ for the oxidation process, comparable to the activation energy obtained from low-temperature oxidation data in the literature [102]. 
Table 5.1. Experimental parameters and fitting results

\begin{tabular}{|c|c|c|c|c|c|c|}
\hline Cell & $T(\mathrm{~K})$ & $\begin{array}{c}h_{\mathrm{Zr}} \\
(\mathrm{nm})\end{array}$ & $\begin{array}{c}h_{\mathrm{ZrO} 2} \\
(\mathrm{~nm})\end{array}$ & $\begin{array}{c}C_{\mathrm{i}} \times 10^{8} \\
(\mathrm{~J} / \mathrm{K})\end{array}$ & $\begin{array}{c}K_{\mathrm{c}} \times 10^{15} \\
\left(\mathrm{~J}^{2} / \mathrm{K}^{2} / \mathrm{s}\right)\end{array}$ & $\begin{array}{c}K \times 10^{15} \\
\left(\mathrm{~m}^{2} / \mathrm{s}\right)\end{array}$ \\
\hline 1 & 660 & 175 & 271 & 2.0 & 0.63 & 0.37 \\
\hline 2 & 710 & 204 & 317 & 4.5 & 1.2 & 0.72 \\
\hline 3 & 760 & 202 & 313 & 2.9 & 1.9 & 1.2 \\
\hline 4 & 781 & 220 & 341 & 1.0 & 3.3 & 2.0 \\
\hline 5 & 835 & 183 & 284 & 1.4 & 5.6 & 3.3 \\
\hline 6 & $360-840$ & 211 & 327 & - & - & - \\
\hline
\end{tabular}
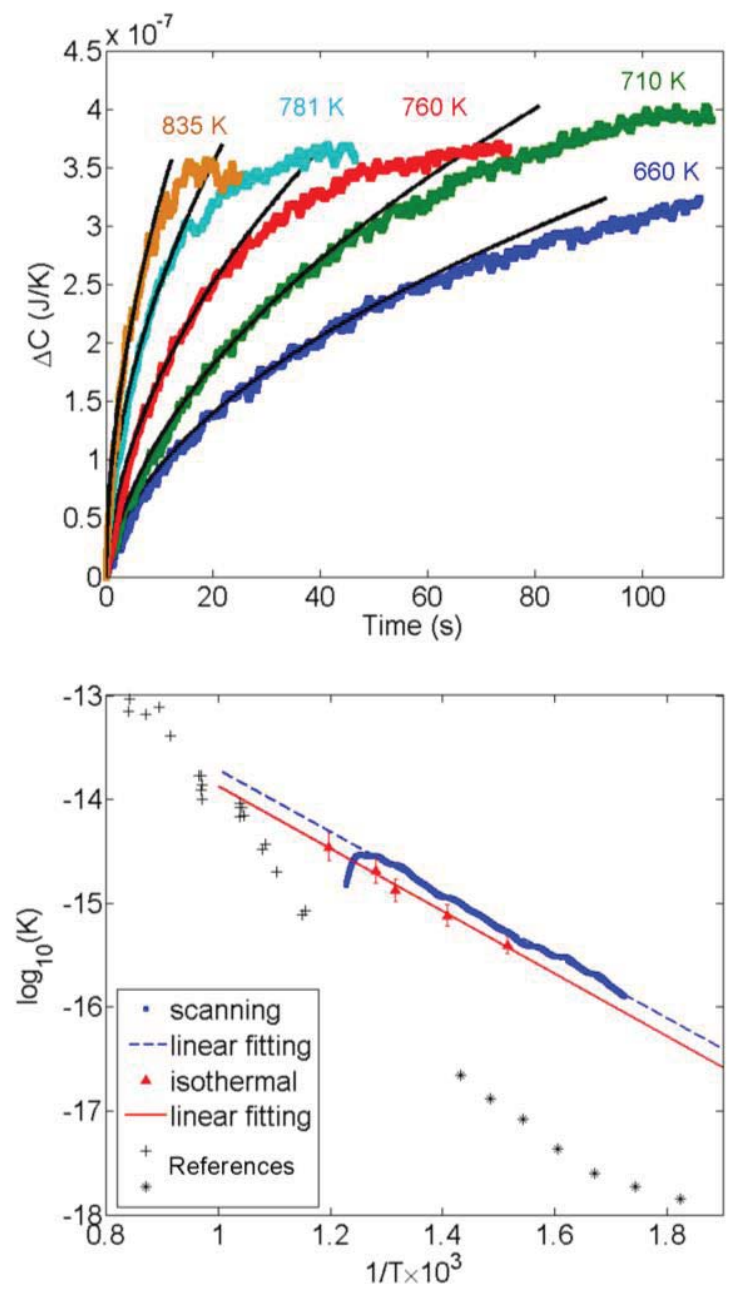

Figure 5.3. (top) Change in heat capacity as a function of time for five different isothermal oxidation experiments; the solid lines represent fits of Eq. (5.5) to the experimental data. (bottom) Logarithm of the parabolic constant $K$ as a function of reciprocal temperature for the isothermal (triangles) and scanning (squares) measurements, along with linear least squares fits and data from references [104] (plus) and

$$
\text { [102] (star). }
$$


If the oxidation reaction is diffusion limited, it is also possible to quantify the reaction kinetics by performing several temperature scans on a single sample, instead of performing isothermal measurements on several different samples [106]. This approach is demonstrated in Figure 5.4, where three different temperature scans are shown for a single $\mathrm{Zr}$ sample: Scan 1 was performed in vacuum, while scans 2 and 3 were performed in air. Scan 1 provides the heat capacity of the unreacted $\mathrm{Zr}$ sample as a function of temperature; scan 2 shows how the heat capacity of the sample changes as the sample oxidizes during the temperature scan; scan 3 provides the heat capacity of the fully oxidized sample. Scan 2 overlaps well with the scan 1 below $500 \mathrm{~K}$, then rises above scan 1 to join scan 3 at $810 \mathrm{~K}$. The overlap between scans 1 and 2 at low temperature indicates that the $\mathrm{Zr}$ film is well protected by its native oxide and that there is no appreciable oxide growth in this temperature range. The native oxide thickness is estimated to be $2-5 \mathrm{~nm}$ [107], less than $2 \%$ of the total oxide thickness, $h_{z r O 2}$, and is neglected in the following analysis. The overlap between scans 2 and 3 at high temperature indicates that the sample is fully oxidized at the end of the second scan, in agreement with the XRD results. The fraction of the $\mathrm{Zr}$ sample that has been oxidized in scan 2 is then calculated from

$$
\frac{\Delta h}{h_{\mathrm{ZrO}_{2}}}=\frac{C_{2}-C_{1}}{C_{3}-C_{1}},
$$

where $C_{1}, C_{2}$ and $C_{3}$ are the experimental heat capacities from scans 1 though 3 , 
respectively. The thickness of the oxide scale and the oxidation rate can be calculated from scan 2 using Eq.(5.6). The results are plotted as a function of temperature in Figure 5.4(b). According to Eq. (5.3), the parabolic rate coefficient $K$ is given by $\Delta h \times \mathrm{d} \Delta h / \mathrm{d} t$. An Arrhenius graph of $K$ obtained from the scanning measurement is shown in Fig 5.3(b), along with the results from the isothermal measurements. The figure demonstrates a linear relationship over the temperature range from $550 \mathrm{~K}$ to $800 \mathrm{~K}$. The graph is also in good agreement with the isothermal measurements, yielding an activation energy $E_{a}=0.59 \pm 0.03 \mathrm{eV}$. This agreement validates the scanning AC nano-calorimetry approach to evaluate reaction kinetics using a single sample. 


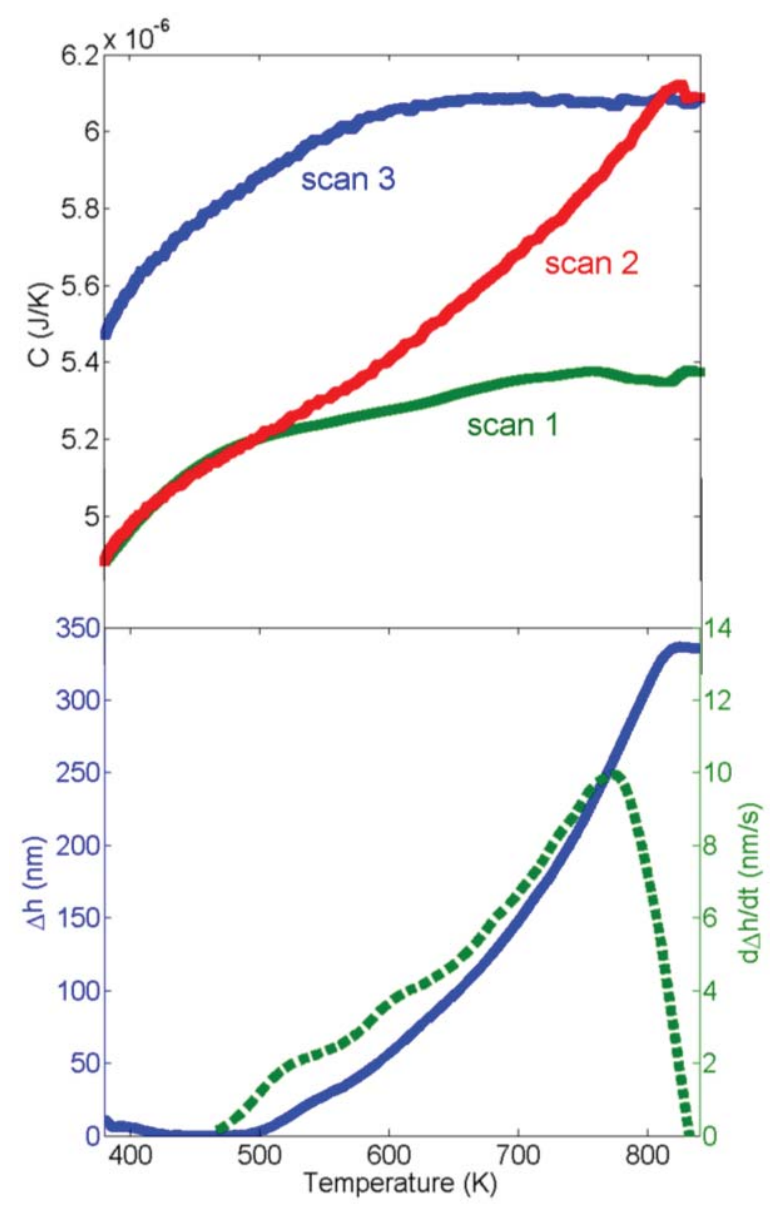

Figure 5.4: (top) Results of scanning measurements in vacuum (scan 1) and air (scans 2 and 3). Scan 1 was performed at a rate of $700 \mathrm{~K} / \mathrm{s}$ to minimize sample oxidation during the reference scan. Scans 2 and 3 were performed at $5 \mathrm{~K} / \mathrm{s}$ to ensure that the temperature distribution was similar to that in the isothermal measurements [22]. The heat capacity of the addendum from the calorimeter is included in all three measurements. (bottom) Graphs of $\Delta h$ and $\mathrm{d} \Delta h / \mathrm{d} t$ derived from the experimental data using Eq. (5.7).

\subsection{Summary}

While isothermal measurements may be required to establish the mechanism controlling the reaction rate, each isothermal measurement provides the rate coefficient at a single temperature. Establishing the temperature dependence of the reaction 
requires many measurements at different temperatures. In contrast, scanning measurements on a single sample allow direct extraction of the rate coefficient as a function of temperature, thus providing an efficient method of evaluating reaction kinetics. We anticipate that this feature will make the scanning technique an attractive choice for fast screening of materials. For instance, the oxidation resistance of an entire alloy system can be determined by performing scanning $A C$ measurements on a composition spread synthesized using magnetron sputtering - something the PnSC device is ideally suited for because of its array design [108-110]. More generally, one can envision using this approach to characterize a broad range of solid-gas phase reactions. 


\section{Chapter 6}

\section{Conclusions and outlook}

This thesis presents an AC nanocalorimetry technique that enables calorimetry measurements on very small quantities of materials over a wide range of scanning rates

(isothermal- $10^{3} \mathrm{~K} / \mathrm{s}$ ), temperatures (ambient to $1500 \mathrm{~K}$ ), and environments (vacuum or reactive). We have presented an analysis of AC calorimetry specified to the PnSC device and have derived conditions under which valid scanning AC measurements can be obtained. The thesis also applies the technique in three distinct and promising fields of materials study:

1. In-situ X-ray diffraction during calorimetry measurements;

The wide working range of scanning $A C$ nanocalorimetry technique makes it possible to be combined with in-situ X-ray diffraction measurements, allowing simultaneous structural and thermal characterization of the samples as a function of temperature. Nanocalorimetry and in-situ XRD provide complementary information on the phase transformations that occur in a sample. We anticipate that the technique will be most useful in the analysis of complex materials systems where identification of features in the calorimetry trace is often not a trivial exercise.

2. Study of reactions over a wide range of scanning rates spanning over 4 orders of 
magnitudes;

Combining both scanning $A C$ and $D C$ nanocalorimetry, a wide range of scanning rates are achieved for calorimetry measurements. When applied to the study of the nucleation reaction in $\mathrm{Bi}$ thin-film samples, we demonstrate that the classical nucleation theory is valid at cooling rates ranging from $10^{\wedge} 1$ to $10^{\wedge} 4 \mathrm{~K} / \mathrm{s}$. The capability to make measurements at widely varying scanning rates is helpful for the study of reaction kinetics, or materials not in equilibrium, e.g. glass transition reactions for polymers or metallic glasses.

3. Thin-film solid-gas reaction at elevated temperatures;

By measuring the change in heat capacity of a sample during reaction using the technique, we evaluate the oxidation kinetics of thin-film samples of zirconium in air. Establishing the temperature dependence of the reaction requires many isothermal measurements at different temperatures. Meanwhile, scanning measurements on a single sample allow direct extraction of the rate coefficient as a function of temperature, thus providing an efficient method of evaluating reaction kinetics. We anticipate that this feature will make the scanning technique an attractive choice for fast screening of materials. For instance, the oxidation resistance of an entire alloy system can be determined by performing scanning AC measurements on a composition spread synthesized using magnetron sputtering - something the PnSC device is ideally suited for 
because of its array design [108-110]. More generally, one can envision using this approach to characterize a broad range of solid-gas phase reactions.

The dynamic range of scanning AC nanocalorimetry makes it ideal to address a broad range of materials problems and is especially well suited to investigate the kinetics of reactions and phase transformations. Apart from the applications detailed in this thesis, scanning AC nanocalorimetry has also been applied to study the martensitic phase transformation of Fe-Ni system [97], the reaction of $\mathrm{Zr} / \mathrm{B}$ multilayers [81], and the glass transformation of metallic glasses. In an on-going research project, it is also being applied to study the initial stage of the oxidation reaction for ZrB2 based ceramics. We envision that the dynamic range of this technique can be further extended to ultra-high temperatures $(>1500 \mathrm{~K})$ as well as ultra-fast scanning rates $\left(>10^{\wedge} 4 \mathrm{~K} / \mathrm{s}\right)$. The current accessible maximum temperature is limited by the PnSC device, rather than the scanning $\mathrm{AC}$ nanocalorimetry technique. The difference in thermal expansion coefficient between the tungsten heater and the supporting silicon nitride membrane of the calorimeter sensor induces stress inside the membrane at high temperatures, which is the most likely cause for sensor cells to fail. A possible solution is a calorimeter sensor with self-supporting heating element. The current limit in scanning rates is set by the resolution of data acquisition electronics. For measurements at fast scanning rates, high AC frequencies $(>5 \mathrm{kHz})$ need to be used, reducing the amplitude of $2 \omega$ voltage signals 
below the current detection limit. This limit can potentially be relaxed with improved integration of frequency filter or lock-in amplifier, which may further increase the signal to noise ratio. 


\section{Appendix}

\section{Appendix A: Validity of AC measurements (Chapter 2)}

To validate the $A C$ measurements, Figure $A .1$ shows the experimental values of the coefficients $C_{1}$ through $C_{5}$ in conditions (2.28) through (2.32) for a typical AC calorimetry measurement on the Sn sample. $C_{3}$ was obtained by applying a Fourier transform to resistance $R$ and by extracting the phase angle $\varphi_{1}$ from its $1 \omega$ component. The maximum values for $C_{1}$ and $C_{2}$ are both well below 0.01 . The curve for $C_{3}$ peaks near the sample melting temperature with a maximum value of 0.06 , which results in a relative error in the amplitude of the $2 \omega$-signal of approximately $0.2 \%$ at the melting point and much smaller at other temperatures. These results show that assumptions (2.28)-(2.30) in the analysis of the AC signal are readily satisfied. The conditions (2.31) and (2.32) imposed by experimental considerations are also met, with $\mathrm{C}_{4} \approx 20$ and $\mathrm{C}_{5}<0.15 \mathrm{~K}$. Thus the AC measurements and analysis are valid. The largest errors in the measurements arise from uncertainty in the sample mass $(15 \%)$, the temperature uniformity of the sensor (3\%), and the temperature calibration of the heating element (4\%). 


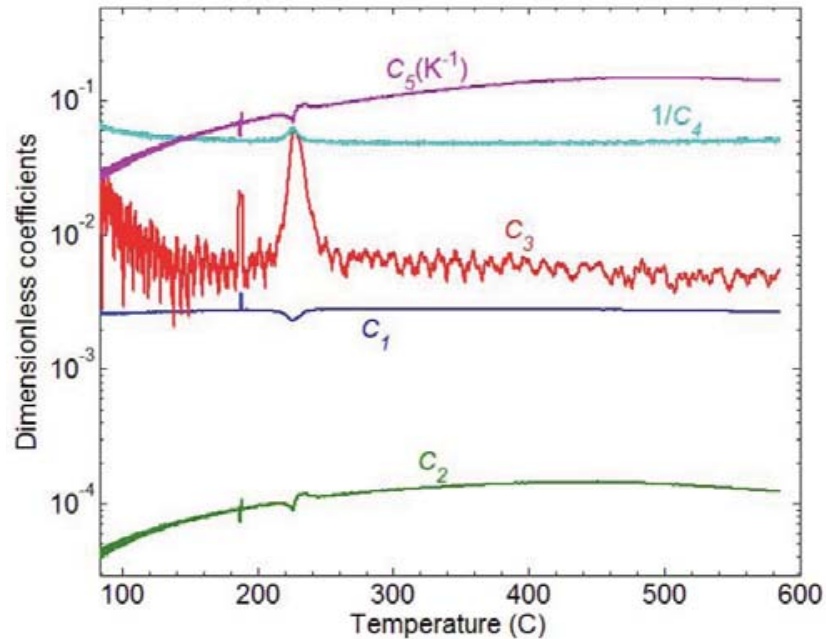

Figure A.1. Experimental results for coefficients $C 1-C 5$. Coefficient $C_{5}$ is given in units of Kelvin. The measurement was performed at a heating rate of $10-10^{2} \mathrm{~K} / \mathrm{s}$ and a frequency of $666.7 \mathrm{~Hz}$ using a sensor with a Sn sample in a He environment. 


\section{Appendix B: Derivation of equations for the $\varphi_{1}$ and $C$ (Chapter 3)}

Dividing Eq. (3.2c) by Eq. (3.2b) and rearranging leads to

$$
\sin ^{2} \varphi_{1}+n \sin \varphi_{1} \cos \varphi_{1}+\frac{1}{4} \sin ^{2} \varphi_{2}+\frac{1}{4} n \sin \varphi_{2} \cos \varphi_{2}=0,
$$

where $n$ is defined by Eq. (3.4). Since $\varphi_{1}$ and $\varphi_{2}$ are in the same quadrant, Eq. (3.3)

implies that

$$
\begin{aligned}
& \sin \varphi_{2}=\frac{2 \sin \varphi_{1}}{\sqrt{1+3 \sin ^{2} \varphi_{1}}} \\
& \cos \varphi_{2}=\frac{\cos \varphi_{1}}{\sqrt{1+3 \sin ^{2} \varphi_{1}}} .
\end{aligned}
$$

Substituting (B2) and (B3) into (B1) yields

$$
\sin ^{2} \varphi_{1}+n \sin \varphi_{1} \cos \varphi_{1}+\frac{\sin ^{2} \varphi_{1}}{1+3 \sin ^{2} \varphi_{1}}+\frac{1}{2} n \frac{\sin \varphi_{1} \cos \varphi_{1}}{1+3 \sin ^{2} \varphi_{1}}=0
$$

Dividing this equation by $\sin \varphi_{1} \cos \varphi_{1}$ yields

$$
\tan \varphi_{1}+n+\frac{\tan \varphi_{1}}{1+3 \sin ^{2} \varphi_{1}}+\frac{1}{2} \frac{n}{1+3 \sin ^{2} \varphi_{1}}=0
$$

Substituting the relationship

$$
\sin ^{2} \varphi_{1}=\frac{\tan ^{2} \varphi_{1}}{1+\tan ^{2} \varphi_{1}}
$$

into (B4) finally leads to the Eq. (3.4) presented in the manuscript.

According to the theorem of third order polynomials, Eq. (3.4) has only one real root uniquely defining the value of $\tan \varphi_{1}$. The value of $\tan \varphi_{2}$ can be calculated using Eq. (3.3). From Eq. (3.2), it follows further that 


$$
\begin{aligned}
& \left|V_{2 \omega}\right|^{2}=X_{2 \omega}^{2}+Y_{2 \omega}^{2} \\
& =\left(\frac{i^{2} I_{0} R_{0}^{2} \lambda}{C \omega}\right)^{2}\left[\sin ^{2} \varphi_{1}+\frac{1}{16} \sin ^{2} \varphi_{2}+\frac{1}{2} \sin \varphi_{1} \sin \varphi_{2}\left(\cos \varphi_{1} \cos \varphi_{2}+\sin \varphi_{1} \sin \varphi_{2}\right)\right]
\end{aligned}
$$

Substituting (B2), (B3), and (B6) into (B7) leads to

$$
\left|V_{2 \omega}\right|^{2}=\left(\frac{i^{2} I_{0} R_{0}^{2} \lambda}{C \omega}\right)^{2} \frac{\tan ^{2} \varphi_{1}}{1+\tan ^{2} \varphi_{1}}\left(\frac{9+25 \tan ^{2} \varphi_{1}}{4+16 \tan ^{2} \varphi_{1}}\right)
$$

which finally leads to Eq. (3.6). 


\section{Appendix C: Data reduction scheme for DC measurements (Chapter 4)}

For a series of $n$ DC calorimetry scans each performed at a different scan rate, the heat balance equations in the absence of any reaction or phase transformation are given

by

$$
\begin{aligned}
& P_{1}=C\left(\frac{d T}{d t}\right)_{1}+L ; \\
& P_{2}=C\left(\frac{d T}{d t}\right)_{2}+L ; \\
& \vdots \\
& P_{n}=C\left(\frac{d T}{d t}\right)_{n}+L,
\end{aligned}
$$

where $P, C$ and $L$ have the same physical meaning as in Eq. (4.2), and the subscripts refer to individual scans. As pointed out previously, both the power supplied to each sensor and its temperature are readily determined as a function of time. Assume that $C$ and $L$ are functions of temperature only, Eq. (C1) can then be considered as a set of linear equations in $C$ and $L$. At each temperature, $C$ and $L$ can be evaluated from a linear fit of $P_{i}$ as a function of $(\mathrm{d} T / \mathrm{d} t)_{i}$, i.e., $C$ and $L$ can be determined as a function of temperature. During a reaction or phase transformation, this linear relationship does not hold. In this case, it may be possible to estimate $C$ and $L$ by interpolation. If heat loss occurs primarily by transient conduction, $L$ depends on the entire temperature history of the sensor and this approach fails.

Figure C.1 depicts the results obtained for the various DC scans in a 1 atm Helium 
environment. Figure 4.6a shows the linear relationship between applied power and scan rate for three representative temperatures, while Figs. C.1b and C.1c show results for $C$ and $L$ over the entire temperature range, except during the phase transformation. It is evident from the figure that the heat capacity of the super-cooled liquid bismuth is slightly larger than that of solid bismuth, in agreement with previous $A C$ nano-calorimetry measurements on thin-film samples [26] and with measurements on bulk samples. The heat loss curve is linear over the temperature range of the experiments, indicating that radiative heat loss is negligible. This result combined with the observation that the heat loss increases with helium pressure suggests convection through the helium as the main heat loss mechanism. 


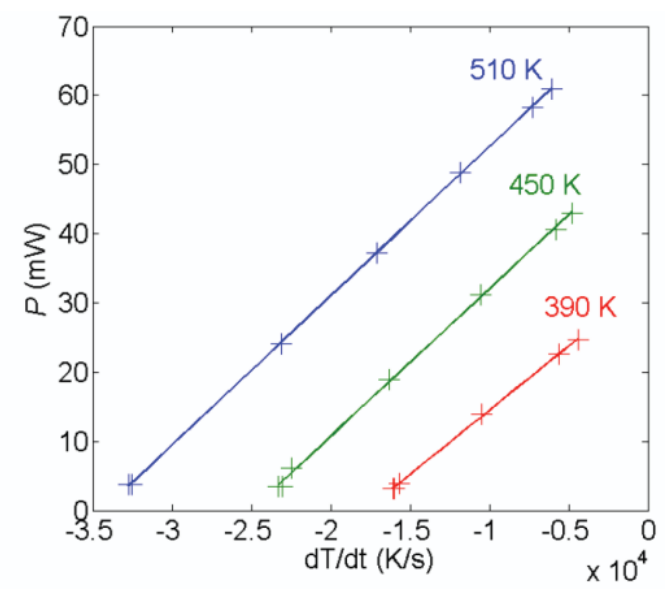

(a)

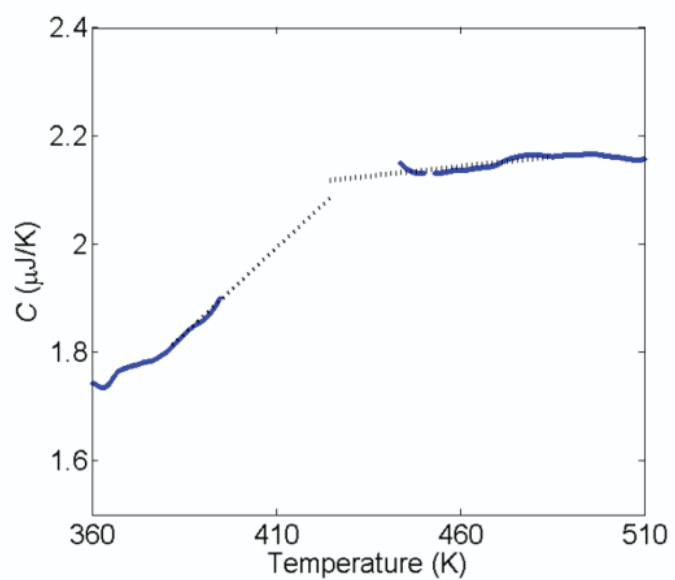

(b)

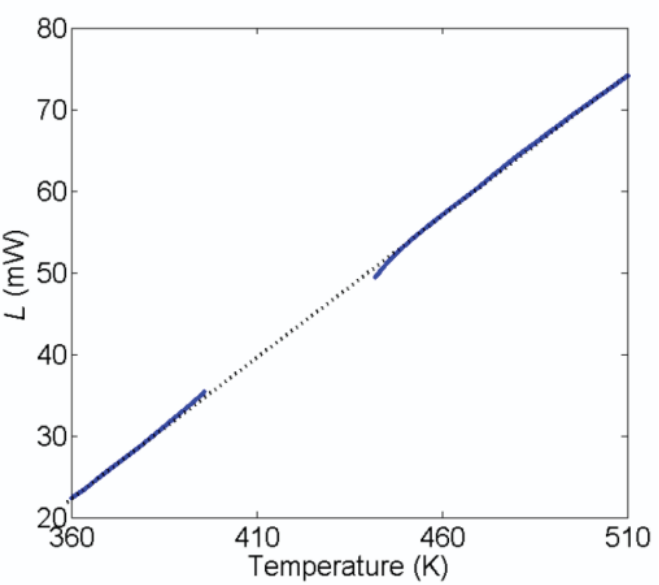

(c)

Figure C.1. a) Applied power as a function of scan rate for three representative temperatures; b) Heat capacity and c) heat loss obtained from a linear fitting of Eq. (C1) at each temperature. 


\section{Appendix D: Effect of size distribution on the nucleation rate (Chapter 4)}

First consider the case of bulk nucleation, where Eq. (4.21) holds true. When the number of droplets is large enough, Eqs (4.24) and (4.25) can be written in integral form:

$$
\begin{gathered}
V=\sum n_{i 0} v_{i} \exp \left(-\int_{0}^{t} I_{i} d t\right)=\int_{0}^{+\infty} v \mathrm{e}^{\left(-\int_{0}^{t} J d t\right) v} \frac{d n_{0}}{d v} d v=\int_{0}^{+\infty} v \mathrm{e}^{-M v} \frac{d n_{0}}{d v} d v \\
\frac{d V}{d t}=-\sum_{i=1}^{m} n_{i 0} v_{i} I_{i} \exp \left(-\int_{0}^{t} I_{i} d t\right)=-\int_{0}^{+\infty} J v^{2} \mathrm{e}^{\left(-\int_{0}^{t} J d t\right) v} \frac{d n_{0}}{d v} d v=-\int_{0}^{+\infty} J v^{2} \mathrm{e}^{-M v} \frac{d n_{0}}{d v} d v,
\end{gathered}
$$

where

$$
M(t)=\int_{0}^{t} J d t
$$

and where $\mathrm{d} n_{0} / \mathrm{d} v$ is the probability density function of the droplet volume at $t=0$ (before any nucleation events). For simplicity, we assume $\mathrm{d} n_{0} / \mathrm{d} v$ is described by a Gaussian distribution,

$$
\frac{d n_{0}}{d v}=\frac{N_{0}}{\sqrt{2 \pi} \sigma} e^{-\frac{\left(v-v_{u}\right)^{2}}{2 \sigma^{2}}}
$$

where $N_{0}$ is the total number of droplets, $v_{\mathrm{u}}$ is the average volume and $\sigma$ the standard deviation at time $t=0$. Substituting Eq. (D4) into (D1) and (D2), we obtain

$$
\begin{aligned}
& V=\frac{N_{0}}{\sqrt{2 \pi} \sigma} \int_{0}^{+\infty} v \mathrm{e}^{-M v} e^{-\frac{\left(v-v_{u}\right)^{2}}{2 \sigma^{2}}} d v \approx \frac{N_{0}}{\sqrt{2 \pi} \sigma} \int_{-\infty}^{+\infty} v \mathrm{e}^{-M v} e^{-\frac{\left(v-v_{u}\right)^{2}}{2 \sigma^{2}}} d v, \\
& \frac{d V}{d t}=-\frac{J N_{0}}{\sqrt{2 \pi} \sigma} \int_{0}^{+\infty} v^{2} \mathrm{e}^{-M v} e^{-\frac{\left(v-v_{u}\right)^{2}}{2 \sigma^{2}}} d v \approx-\frac{J N_{0}}{\sqrt{2 \pi} \sigma} \int_{-\infty}^{+\infty} v^{2} \mathrm{e}^{-M v} e^{-\frac{\left(v-v_{u}\right)^{2}}{2 \sigma^{2}}} d v .
\end{aligned}
$$

The last approximation is accurate as long as $V_{\mathrm{u}}>3 \sigma$. The integrals in Eqs. (D5) and (D6) 
can be evaluated analytically

$$
\begin{aligned}
& V=\frac{N_{0}}{\sqrt{2 \pi} \sigma} \int_{-\infty}^{+\infty} v \mathrm{e}^{-M v} e^{-\frac{\left(v-v_{u}\right)^{2}}{2 \sigma^{2}}} d v=N_{0}\left(v_{u}-\sigma^{2} M\right) e^{-M v_{u}+\frac{1}{2} M^{2} \sigma^{2}} \\
& =V_{0}\left(1-\frac{\sigma^{2} M}{v_{u}}\right) e^{-M v_{u}+\frac{1}{2} M^{2} \sigma^{2}} \\
& \frac{d V}{d t}=-\frac{J N_{0}}{\sqrt{2 \pi} \sigma} \int_{-\infty}^{+\infty} v^{2} \mathrm{e}^{-M v} e^{-\frac{\left(v-v_{u}\right)^{2}}{2 \sigma^{2}}} d v=-J N_{0} e^{-M v_{u}+\frac{1}{2} M^{2} \sigma^{2}}\left[\sigma^{2}+\left(v_{u}{ }^{2}-\sigma^{2} M\right)^{2}\right] \\
& =-I V_{0} e^{-M v_{u}+\frac{1}{2} M^{2} \sigma^{2}}\left[\frac{\sigma^{2}}{v_{u}^{2}}+\left(1-\frac{\sigma^{2} M}{v_{u}}\right)^{2}\right]
\end{aligned}
$$

Note that if $\sigma=0$, then Eqs. (D7) and (D8) are identical to Eqs. (4.26) and (4.27). We can thus calculate the ratio between the volume rates $d V / d t$ calculated from the full size distribution and from the average size:

$$
\eta=\frac{d V}{d t} /\left.\left(\frac{d V}{d t}\right)\right|_{\sigma=0}=e^{\frac{1}{2} M^{2} \sigma^{2}}\left[\frac{\sigma^{2}}{v_{u}^{2}}+\left(1-\frac{\sigma^{2}}{v_{u}} M\right)^{2}\right] .
$$

For simplicity, we take $M=0.7 / v_{u}$ for a conversion rate of approximately $50 \%$, which is used in the analysis of the experiments

$$
1-\frac{V}{V_{0}}=1-\left(1-\frac{\sigma^{2}}{v_{u}^{2}}\right) e^{-0.7+0.245 \sigma^{2} / v_{u}^{2}} \approx 1-e^{-0.7} \approx 50 \% \text {. }
$$

Substituting this value of $M$ into Eq. (D9) yields

$$
\eta=e^{0.245\left(\frac{\sigma}{v_{u}}\right)^{2}}\left[\left(\frac{\sigma}{v_{u}}\right)^{2}+\left(1-0.7\left(\frac{\sigma}{v_{u}}\right)^{2}\right)^{2}\right]=e^{0.245 \beta}\left[1-0.4 \beta+0.49 \beta^{2}\right],
$$

where $\beta=\left(\sigma / v_{\mathrm{u}}\right)^{2}$ is a parameter describing the width of the volume distribution relative to its mean. A smaller value of $\beta$ indicates a tighter distribution. Equation (D11) provides the error associated with using the average instead of the full size distribution. Figure 
D. 1 shows a plot $\eta$ as a function of $\beta$. In general, $\eta$ is larger than 0.98 , i.e., using the average droplet size causes an error of less than $2 \%$ in the nucleation rate. In this study, $\beta \sim 0.1$

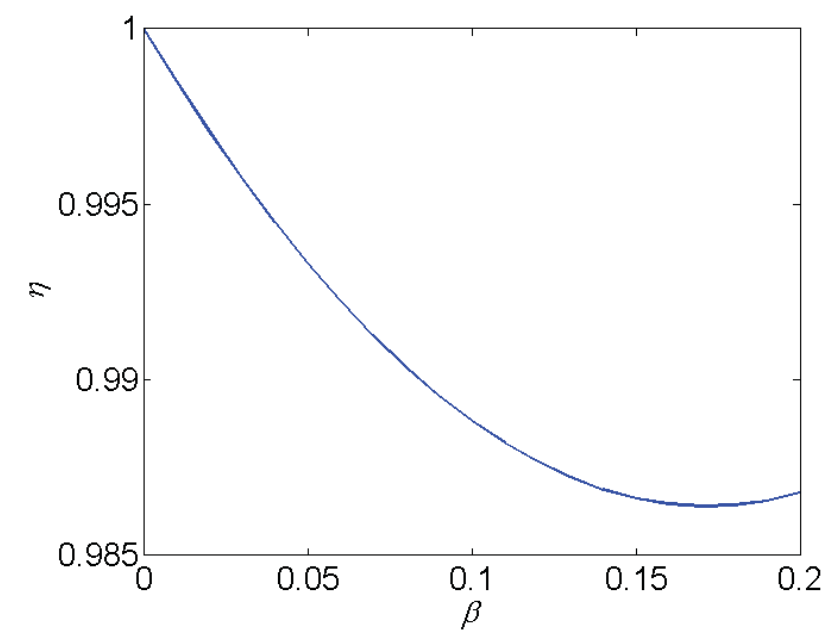

Figure D.1. $\eta$ as a function of $B$ at $M=0.7 / V_{u}$.

For the case of interface nucleation, the ratio between the volume rates for groups $i$ and $j$ is (assume $v_{\mathrm{i}}>v_{\mathrm{j}}$ ):

$$
\frac{d n_{i}}{d t} v_{i} /\left(\frac{d n_{j}}{d t} v_{j}\right)=\frac{v_{i} s_{i}}{v_{j} s_{j}}<\left(\frac{v_{i}}{v_{j}}\right)^{2}
$$

The right hand side is the ratio of the volume rates in the case of bulk nucleation. This indicates that the distribution of the volume rates in the case of interface nucleation is tighter than for bulk nucleation. Thus, in the case of interface nucleation, $\eta$ should be closer to unity than the value given by Eq. (D19).

This analysis shows that the effect of the droplet size distribution on the analysis is small as long as the ratio of the standard deviation of the distribution to its mean is 
small. Furthermore, it is evident from Eqs. (D7) and (D9) that the value of $\eta$ at a given conversion fraction $\left(1-V / V_{0}\right)$ is only a function of $v_{\mathrm{u}}$ and $\sigma$, and is independent of the temperature history. This implies that for a given size distribution, all measurements need to be corrected by the same factor if the average size model is used. Thus the slope of the data in Figure $4.5 \mathrm{~b}$ is unaffected, while the intersect needs to be corrected by a corresponding amount.

To demonstrate how well the above analysis works in our specific case, we compare the solidification rates of two sets of Bi droplets using the data in Table 4.1: a set of droplets that follow the experimental size distribution (Figure $4.2 \mathrm{~b}$ ) where the solidification rate is given by Eq. (4.25), and a set of droplets of uniform size where the solidification rate is given by Eq. (4.27). The results are depicted in Figure D.2. It is obvious from the figure that the effect of the precise size distribution is comparable to the analysis result from Eq. (D11), and is indeed negligible: a $0.5 \mathrm{~K}$ difference in peak temperature and a $2 \%$ difference in peak height. 


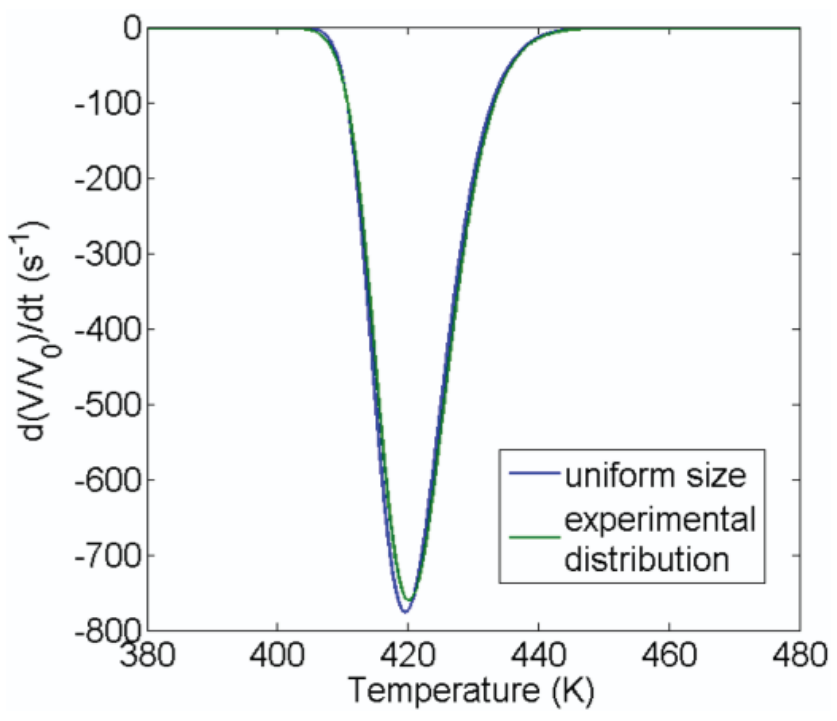

Figure D.2. Volumetric solidification rate normalized by the initial volume as a function of temperature for droplets that follow the experimental distribution and that are uniform in size. The time-temperature trace of the fastest DC scan is used in the calculation. 


\section{Bibliography}

1. G. Höhne, W. Hemminger and H.-J. Flammersheim, Differential scanning calorimetry. (Springer Science \& Business Media, 2003).

2. D. Denlinger, E. Abarra, K. Allen, P. Rooney, M. Messer, S. Watson and F. Hellman, Review of Scientific Instruments 65 (4), 946-959 (1994).

3. S. Lai, G. Ramanath, L. Allen, P. Infante and Z. Ma, Applied Physics Letters 67, 1229 (1995).

4. S. L. Lai, J. Y. Guo, V. Petrova, G. Ramanath and L. H. Allen, Physical Review Letters 77 (1), 99-102 (1996).

5. A. A. Minakov and C. Schick, Review of Scientific Instruments 78 (7), 073902 (2007).

6. A. A. Minakov, Y. V. Bugoslavsky and C. Schick, Thermochimica Acta 317 (2), 117-131 (1998).

7. P. J. McCluskey and J. J. Vlassak, Journal of Materials Research 25 (11), 2086-2100 (2010).

8. A. F. Lopeandía, J. Rodríguez-Viejo, M. Chacón, M. T. Clavaguera-Mora and F. J. Muñoz, Journal of Micromechanics and Microengineering 16 (5), 965 (2006).

9. A. A. Minakov, S. A. Adamovsky and C. Schick, Thermochimica Acta 432 (2), 177-185 (2005).

10. B. Revaz, B. Zink and F. Hellman, Thermochimica Acta 432 (2), 158-168 (2005).

11. E. Zhuravlev and C. Schick, Thermochimica Acta 505 (1), 1-13 (2010).

12. E. Zhuravlev and C. Schick, Thermochimica Acta 505 (1), 14-21 (2010).

13. S. Lai, G. Ramanath, L. Allen and P. Infante, Applied Physics Letters 70, 43 (1997).

14. M. Y. Efremov, E. A. Olson, M. Zhang, F. Schiettekatte, Z. Zhang and L. H. Allen, Review of Scientific Instruments 75 (1), 179-191 (2004).

15. M. Y. Efremov, J. Warren, E. Olson, M. Zhang, A. Kwan and L. Allen, Macromolecules 35 (5), 1481-1483 (2002).

16. A. Minakov, S. Adamovsky and C. Schick, Thermochimica Acta 432 (2), 177-185 (2005). 
17. A. Lacey, D. Price and M. Reading, Modulated Temperature Differential Scanning Calorimetry, 1-81 (2006).

18. P. Handler, D. Mapother and M. Rayl, Physical Review Letters 19 (7), 356-358 (1967).

19. P. F. Sullivan and G. Seidel, Physical Review 173 (3), 679-685 (1968).

20. Y. Kraftmakher, Physics Reports 356 (1), 1-117 (2002).

21. H. S. Carslaw and J. C. Jaeger, Conduction of heat in solids. (Clarendon Press Oxford, 1959).

22. K. Xiao, J. M. Gregoire, P. J. McCluskey and J. J. Vlassak, Review of Scientific Instruments $\mathbf{8 3}$ (11), 114901 (2012).

23. P. J. McCluskey and J. J. Vlassak, Thin Solid Films 518 (23), 7093-7106 (2010).

24. K. Xiao and J. J. Vlassak, Thermochimica Acta 603 (0), 29-38 (2015).

25. K. Xiao, D. Lee and J. J. Vlassak, Applied Physics Letters 105 (17), 171901 (2014).

26. K. Xiao, J. M. Gregoire, P. J. McCluskey, D. Dale and J. J. Vlassak, Journal of Applied Physics 113 (24), 243501 (2013).

27. H. E. Kissinger, Analytical Chemistry 29 (11), 1702-1706 (1957).

28. J. Zhang and Y. Zhao, Nature 430 (6997), 332-335 (2004).

29. J. M. Gregoire, P. J. McCluskey, D. Dale, S. Ding, J. Schroers and J. J. Vlassak, Scripta Materialia 66 (3), 178-181 (2011).

30. T. F. J. Pijpers, V. B. F. Mathot, B. Goderis, R. L. Scherrenberg and E. W. van der Vegte, Macromolecules 35 (9), 3601-3613 (2002).

31. H. Huth, A. A. Minakov and C. Schick, Journal of Polymer Science Part B: Polymer Physics 44 (20), 2996-3005 (2006).

32. A. Minakov, J. Morikawa, T. Hashimoto, H. Huth and C. Schick, Measurement Science and Technology 17 (1), 199-207 (2006).

33. L. A. Rosenthal, Review of Scientific Instruments 32 (9), 1033-1036 (1961).

34. L. R. Holland, Journal of Applied Physics 34 (8), 2350-2357 (1963).

35. C. Dames and G. Chen, Review of Scientific Instruments 76 (12), 124902-124902-124914 
(2005).

36. O. M. Corbino, Physik Zeitschrift XI, 413 (1910).

37. H. Petersen, The Properties of Helium: Density, Specific Heats, Viscosity, and Thermal Conductivity at Pressures from 1 to 100 Bar and from Room Temperature to about $1800 \mathrm{~K}$. (Jul. Gjellerup, 1970).

38. T. C. Totemeier and C. J. Smithells, Smithells metals reference book. (Butterworth-Heinemann, 2004).

39. R. C. Weast, M. J. Astle and W. H. Beyer, CRC handbook of chemistry and physics. (CRC press Boca Raton, FL, 1988).

40. C. H. Mastrangelo, Y. C. Tai and R. S. Muller, Sensors and Actuators A: Physical 23 (1-3), 856-860 (1990).

41. M. Y. Efremov, E. A. Olson, M. Zhang, Z. Zhang and L. H. Allen, Macromolecules 37 (12), 4607-4616 (2004).

42. G. Goertzel, The American Mathematical Monthly 65 (1), 34-35 (1958).

43. A. Savitzky and M. J. E. Golay, Analytical chemistry 36 (8), 1627-1639 (1964).

44. B. J. McBride, S. Gordon and M. A. Reno, in NASA TP-3287-REV1 (2001).

45. S. L. Simon, Thermochimica Acta 374 (1), 55-71 (2001).

46. P. R. Garnier and M. B. Salamon, Physical Review Letters 27 (22), 1523-1526 (1971).

47. Y. Saruyama, Journal of Thermal Analysis and Calorimetry 38 (8), 1827-1833 (1992).

48. M. Y. Efremov, E. A. Olson, M. Zhang, S. L. Lai, F. Schiettekatte, Z. S. Zhang and L. H. Allen, Thermochimica Acta 412 (1-2), 13-23 (2004).

49. A. Minakov, S. Roy, Y. Bugoslavsky and L. Cohen, Review of Scientific Instruments 76, 043906 (2005).

50. T. Suzuki, T. Tsuboi and H. Takaki, Japanese Journal of Applied Physics 21 (2), 368 (1982).

51. J. W. Elmer and E. D. Specht, Journal of Electronic Materials 40 (2), 201-212 (2011).

52. M. Y. Efremov, F. Schiettekatte, M. Zhang, E. Olson, A. Kwan, R. Berry and L. Allen, Physical 
Review Letters 85 (17), 3560-3563 (2000).

53. M. Y. Efremov, E. A. Olson, M. Zhang, Z. Zhang and L. H. Allen, Physical Review Letters 91 (8), 85703 (2003).

54. W. Chen, D. Zhou, G. Xue and C. Schick, Frontiers of Chemistry in China 4 (3), 229-248 (2009).

55. P. J. McCluskey and J. J. Vlassak, Scripta Materialia 64 (3), 264-267 (2011).

56. P. J. McCluskey, C. Zhao, O. Kfir and J. J. Vlassak, Acta Materialia 59 (13), 5116-5124 (2011).

57. G. Guenther, E. Aulbach, H. Hahn and O. Guillon, Thermochimica Acta 522 (1), 77-85 (2011).

58. J. M. Gregoire, D. Dale, A. Kazimirov, F. J. DiSalvo and R. B. van Dover, Review of Scientific Instruments 80 (12), 123905 (2009).

59. M. Kano, Journal of Physics E: Scientific Instruments 22 (11), 907 (1989).

60. B. Yang, Y. Gao, C. Zou, Q. Zhai, A. S. Abyzov, E. Zhuravlev, J. W. P. Schmelzer and C. Schick, Applied Physics A 104 (1), 189-196 (2010).

61. B. Yang, A. Abyzov, E. Zhuravlev, Y. Gao, J. Schmelzer and C. Schick, Journal of Chemical Physics 138, 054501 (2013).

62. S. Hishita, Z. Stryhal, I. Sakaguchi, N. Ohashi, N. Saito and H. Haneda, Thin Solid Films 464465 (0), 146-149 (2004).

63. G. Wilde, C. Santhaweesuk, J. Sebright, J. Bokeloh and J. Perepezko, Journal of Physics: Condensed Matter 21 (46), 464113 (2009).

64. G. Wilde, J. Sebright and J. Perepezko, Acta Materialia 54 (18), 4759-4769 (2006).

65. S. Klein, D. Holland-Moritz and D. M. Herlach, Physical Review B 80 (21), 212202 (2009).

66. M. Uttormark, J. Zanter and J. Perepezko, Journal of crystal growth 177 (3), 258-264 (1997).

67. E. A. Gehan, Journal of chronic diseases 21 (9), 629 (1969).

68. D. Turnbull and B. Vonnegut, Industrial \& Engineering Chemistry 44 (6), 1292-1298 (1952).

69. D. Turnbull, Journal of Chemical Physics 18 (2), 198-203 (1950).

70. D. Turnbull, Journal of Chemical Physics 20 (3), 411-424 (1952). 
71. J. Perepezko and J. Paik, Journal of non-crystalline solids 61, 113-118 (1984).

72. J. H. Perepezko, Materials Science and Engineering 65 (1), 125-135 (1984).

73. D. Turnbull, Journal of Applied Physics 21 (10), 1022-1028 (1950).

74. B. Vonnegut, Journal of colloid science 3 (6), 563-569 (1948).

75. D. Turnbull, Journal of Chemical Physics 18, 768-769 (1950).

76. D. Turnbull and R. E. Cech, Journal of Applied Physics 21 (8), 804-810 (1950).

77. J. Perepezko, Materials Science and Engineering: A 226, 374-382 (1997).

78. S. Chaubey, V. Singh and P. Ramachandrarao, Bulletin of Materials Science 9 (4), 277-285 (1987).

79. V. P. Skripov, Metastable liquids. (Wiley, 1974).

80. B. Yang, Y. Gao, C. Zou, Q. Zhai, E. Zhuravlev and C. Schick, Chinese Science Bulletin 55 (19), 2063-2065 (2010).

81. D. Lee, G.-D. Sim, K. Xiao, Y. S. Choi and J. J. Vlassak, Journal of Applied Physics 114 (21), 214902 (2013).

82. E. Olson, M. Y. Efremov, M. Zhang, Z. Zhang and L. Allen, Journal of Applied Physics 97 (3), 034304 (2005).

83. S. Vyazovkin, A. K. Burnham, J. M. Criado, L. A. Pérez-Maqueda, C. Popescu and N. Sbirrazzuoli, Thermochimica Acta 520 (1), 1-19 (2011).

84. D. Turnbull, Trans. AIME, J. Metals 188, 1144-1148 (1950).

85. J. J. Hoyt and M. Asta, Physical Review B 65 (21), 214106 (2002).

86. F. Spaepen, SOLID STATE PHYSICS-NEW YORK-ACADEMIC PRESS- 47, 1-1 (1994).

87. W. Yoon, J. S. Paik, D. LaCourt and J. H. Perepezko, Journal of Applied Physics 60 (10), 3489-3494 (1986).

88. E. J. Szekely J, Sohn HY, Gas-solid reactions. (Academic Press Inc, New York, 1976).

89. G. Wranglén, An introduction to corrosion and protection of metals. (Chapman and Hall Ltd., London, 1985). 
90. T. Yamashita, G. Chen, J. Shir and T. Chen, Magnetics, IEEE Transactions on 24 (6), 2629-2634 (1988).

91. A. L. Rudd, C. B. Breslin and F. Mansfeld, Corrosion Science 42 (2), 275-288 (2000).

92. M. Leskelä, T. Leskelä and L. Niinistö, Journal of Thermal Analysis 40 (3), 1077-1088 (1993).

93. J. Lin, B. Mishra, J. Moore and W. Sproul, Surface and Coatings Technology 202 (14), 3272-3283 (2008).

94. S. Lai, J. Guo, V. Petrova, G. Ramanath and L. Allen, Physical Review Letters 77 (1), 99 (1996).

95. S. Lai, J. Carlsson and L. Allen, Applied Physics Letters 72 (9), 1098-1100 (1998).

96. M. Zhang, M. Y. Efremov, F. Schiettekatte, E. Olson, A. Kwan, S. Lai, T. Wisleder, J. Greene and L. Allen, Physical Review B 62 (15), 10548 (2000).

97. J. M. Gregoire, K. Xiao, P. J. McCluskey, D. Dale, G. Cuddalorepatta and J. J. Vlassak, Applied Physics Letters 102 (20), 201902 (2013).

98. P. D. File, The International Centre for Diffraction Data (ICDD) 12 (2005).

99. M. W. Chase and J. A. N. A. Force, (1998).

100. B. Cox, in Advances in Corrosion Science and Technology (Springer, 1976), pp. 173-391.

101. K. R. Lawless, Reports on Progress in Physics 37 (2), 231 (1974).

102. E. A. Gulbransen and K. F. Andrew, Trans AIME 185, 515-525 (1949).

103. W. M. Haynes, CRC handbook of chemistry and physics. (CRC press, 2012).

104. D. Cubicciotti, Journal of the American Chemical Society 72 (9), 4138-4141 (1950).

105. B. Cox and J. Pemsler, Journal of Nuclear Materials 28 (1), 73-78 (1968).

106. R. Highmore, J. Evetts, A. Greer and R. Somekh, Applied Physics Letters 50 (10), 566-568 (1987).

107. U. Evans, The Corrosion and Oxidation of Metals. (Edward Arnold, London, 1960).

108. J. M. Gregoire, P. J. McCluskey, D. Dale, S. Ding, J. Schroers and J. J. Vlassak, Scripta Materialia 66 (3), 178-181 (2012). 
109. P. J. McCluskey, K. Xiao, J. M. Gregoire, D. Dale and J. J. Vlassak, Thermochimica Acta (2014).

110. Y. Motemani, P. J. McCluskey, C. Zhao, M. J. Tan and J. J. Vlassak, Acta Materialia 59 (20), 7602-7614 (2011). 